\title{
On Defining Relations of Affine Lie Superalgebras and Affine Quantized Universal Enveloping Superalgebras
}

\author{
By \\ Hiroyuki YAMANE *

\section{Introduction}

0.1. In this paper, we give defining relations satisfied by the Chevalley generators $H \in \mathscr{H}, E_{i}, F_{i}(0 \leq i \leq n=\operatorname{rank} \mathscr{G}-1)$ of affine Lie superalgebras $\mathscr{G}$, and defining relations satisfied by the Chevalley generators of affine quantized universal enveloping superalgebras $U_{q}(\mathscr{G})$ except for $U_{q}\left(\mathrm{~A}(m, m)^{(2)}\right)$ and $U_{q}\left(\mathbf{A}(m, m)^{(4)}\right)$. Moreover, for $U_{q}\left(\left(\mathrm{~A}(m, m)^{(1)}\right)^{\mathscr{H}}\right)$ and $U_{q}\left(s l(m, n)^{(1)}\right)(m \neq n)$, we also get defining relations for the Drinfeld realization (see 1.5 for the notation $\left.\left(\mathbf{A}(m, m)^{(1)}\right)^{\mathscr{H}}\right)$.

In 0.3 and 0.5 , we give outlines how we get the defining relations of $\mathscr{G}$ and $U_{q}(\mathscr{G})$.

Part of the results of this paper has been announced in [Y2]. Similar results for finite dimensional simple Lie superalgebras of type A-G have been obtained in $[\mathrm{Y} 1]$.

0.2. Let $(\mathscr{E}, \Pi, p)$ be a triple of a finite dimensional complex linear space $\mathscr{E}$ with a non-degenerate symmetric bilinear form $($,$) , a linearly independent subset \Pi=$ $\left\{\alpha_{0}, \ldots, \alpha_{n}\right\}$ of $\mathscr{E}$, and a function $p: \Pi \rightarrow\{0,1\}$. We call such a triple $(\mathscr{E}, \Pi, p)$ a datum.

We say that a Lie superalgebra $\mathscr{G}^{\#}=\mathscr{G}^{\#}(\mathscr{E}, \Pi, p)$ is admissible with respect to $(\mathscr{E}, \Pi, p)$ if $\mathscr{G}^{\#}$ satisfies the following conditions (see Definition 1.2.1).

(x) $\mathscr{H}=\mathscr{E}^{*}$ is embedded into $\mathscr{G}^{\#}$,

(y) As a Lie superalgebra, $\mathscr{G}^{\#}$ is generated by the generators $\left\{H, E_{i}, F_{i}\right\}$ with parities $p(H)=0, p\left(E_{i}\right)=p\left(F_{i}\right)=p\left(\alpha_{i}\right)$. (We call $\left\{H, E_{i}, F_{i}\right\}$ the Chevalley generators of $\mathscr{G}^{\sharp}$.)

(z) The Chevalley generators of $\mathscr{G}^{\#}$ satisfy

Communicated by M. Kashiwara, April 16, 1996. Revised July 21, 1998.

1991 Mathematics Subject Classifications : 17B37, 16W30, 17B70, 17 B65

* Dept. of Math., Osaka Univ., Toyonaka 560-0043, Japan. 


$$
\begin{gathered}
{\left[H, H^{\prime}\right]=0\left(H, H^{\prime} \in \mathscr{H}\right),\left[H, E_{i}\right]=\alpha_{i}(H) E_{i},\left[H, F_{i}\right]=-\alpha_{i}(H) F_{i}} \\
{\left[E_{i}, F_{j}\right]=\delta_{i j} H_{\alpha_{i}}}
\end{gathered}
$$

where $H_{\alpha_{i}}$ is a unique element of $\mathscr{H}$ such that $\nu\left(H_{\alpha_{i}}\right)=\left(\nu, \alpha_{i}\right)$ for any $\nu \in \mathscr{E}$.

Let $\mathscr{G}^{\#}=\mathscr{G}^{\sharp}(\mathscr{E}, \Pi, p)$ and $\mathscr{G}^{\# \#}=\mathscr{G}^{\# \#}(\mathscr{E}, \Pi, p)$ be admissible with respect to an $(\mathscr{E}, \Pi, p)$. Then we write $\mathscr{G}^{\#}>\mathscr{G}^{\#}$ if there is an epimorphism $\Psi=\Psi\left[\mathscr{G}_{\#}^{\#}, \mathscr{G}^{\#}\right]: \mathscr{G}^{\#}$ $\rightarrow \mathscr{G}^{\#}$ such that $\Psi(H)=H, \Psi\left(E_{i}\right)=E_{i}$ and $\Psi\left(F_{i}\right)=F_{i}$. Then there exists a unique minimal (resp. maximal) one $\mathscr{G}=\mathscr{G}(\mathscr{E}, \Pi, p)$ (resp. $\tilde{\mathscr{G}}=\tilde{\mathscr{G}}(\mathscr{E}, \Pi, p)$ ) with respect to $>$. The Lie superalgebra $\mathscr{G}(\mathscr{E}, \Pi, p)$ is called the Kac-Moody Lie superalgebra.

Van de Leur [VdL1-2] classified Kac-Moody Lie superalgebras of finite growth; they are:

(I) the finite dimensional Kac-Moody Lie superalgebras,

or

(II) the affine Lie superalgebras (see [K2] and 1.5 for the notation below):

$\begin{array}{lll}\text { ( i ) } & s l(m, n-m+1)^{(1)} & (n \geq 2,1 \leq m<n, 2 m \neq n+1), \\ \text { ( ii ) } & \left(\mathrm{A}\left(\frac{n-1}{2}, \frac{n-1}{2}\right)^{(1)}\right)^{\mathscr{H}}(\text { see } 1.5) & (n \geq 3, n=\text { odd), } \\ \text { ( iii) } & s l(2 n-2 m+1,2 m)^{(2)} & (n \geq 1,1 \leq m \leq n), \\ \text { (iv) } & s l(2 m, 2 n-2 m)^{(2)} & (n \geq 3,1 \leq m<n, 2 m \neq n), \\ \text { ( v ) } & \mathrm{A}(n-1, n-1)^{(2)} & (n \geq 4, n=\text { even), } \\ \text { ( vi ) } & s l(2 m+1,2 n-2 m+1)^{(4)} & (n \geq 1,0 \leq m \leq n, 2 m \neq n), \\ \text { (vii) } & \mathrm{A}(n, n)^{(4)} & (n \geq 2, n=\text { even), } \\ \text { (viii) } & \operatorname{osp}(2 n-2 m+1,2 m)^{(1)} & (n \geq 1,1 \leq m \leq n), \\ \text { (ix) } & \operatorname{osp}(2 m, 2 n-2 m)^{(1)} & (n \geq 3,1 \leq m<n), \\ \text { (x) } & \operatorname{osp}(2 m, 2 n-2 m+2)^{(2)} & (n \geq 1,1 \leq m \leq n), \\ \text { (xi) } & \mathrm{D}(2,1 ; x)^{(1)} \quad(x \neq 0,-1) & (n=3), \\ \text { (xii) } & \mathrm{F}(4)^{(1)} & (n=4), \\ \text { (xiii) } & \mathrm{G}(3)^{(1)} & (n=3) .\end{array}$

In $\S 4$ and $\S 5$, we state and prove a Serre-type theorem for the affine Lie superalgebra $\mathscr{G}=\mathscr{G}(\mathscr{E}, \Pi, p)$. In other words, we get defining relations of $\mathscr{G}$ satisfied by the Chevalley generators (Theorem 4.5.1, Theorem 5.1.1, Theorem 5.2.1 and Theorem 5.3.1).

0.3. We are going to give an outline how we get the defining relations of $\mathscr{G}=$ $\mathscr{G}(\mathscr{E}, \Pi, p)$. Let $(\mathscr{E}, \Pi, p)$ be a datum such that $\mathscr{G}=\mathscr{G}(\mathscr{E}, \Pi, p)$ is one of (i )-(xii). To prove our theorems, we associate with each $\mathscr{G}$ a (non-super) affine Lie algebra $\mathscr{G}^{\dagger}$ as in the table below (see 2.4, 5.1 and 5.2; in the text, instead of giving $\mathscr{G}^{\dagger}$, we give only the datum of $\mathscr{G}^{\dagger}$ ). 


\begin{tabular}{|c|c|c|c|c|c|c|}
\hline$g$ & $(\mathrm{i}),(\mathrm{ii})$ & (iii), (viii) & (iv), (v), (ix) & $(\mathrm{vi}),(\mathrm{vii}),(\mathrm{x})$ & $(\mathrm{xi})$ & (xii) \\
\hline$g^{\dagger}$ & $s l(n+1)^{(1)}$ & $s l(2 n+1)^{(2)}$ & $s p(2 n)^{(1)}$ & $s o(2 n+2)^{(2)}$ & $s p(6)^{(1)}$ & $F_{4}{ }^{(1)}$ \\
\hline
\end{tabular}

Let $P^{\dagger}$ be the root lattice of $\mathscr{G}^{\dagger}$. Let $W$ be the Weyl group of $P^{\dagger}$. Then we define an action of $W$ on a set of data $\left(\mathscr{E}^{\prime}, \Pi^{\prime}, p^{\prime}\right)$ such that $\mathscr{G}\left(\mathscr{E}^{\prime}, \Pi^{\prime}, p^{\prime}\right)$ are isomorphic to $\mathscr{G}$ (Definition 3.4.2). For $y \in W$, let $\left({ }^{y} \mathscr{E},{ }^{y} \Pi,{ }^{y} p\right)=y .(\mathscr{E}, \Pi, p)$, and ${ }^{y} \mathscr{G}=$ $\mathscr{G}\left({ }^{y} \mathscr{E},{ }^{y} \Pi,{ }^{y} p\right)$. For the unit element $e$ of $W, e .(\mathscr{E}, \Pi, p)=(\mathscr{E}, \Pi, p)$ and ${ }^{e} \mathscr{G}=\mathscr{G}$. In general, there exists a $y \in W$ such that $\left({ }^{y} \mathscr{E},{ }^{y} \Pi,{ }^{y} p\right)$ is not isomorphic to $(\mathscr{E}, \Pi, p)$ as a datum. Let ${ }^{y} P$ be the root lattice of ${ }^{y} \mathscr{G}$. For each $y \in W$, we fix an identification of ${ }^{y} P$ and $P^{\dagger}$ (see Definition 2.4.1). Then we show that, for $w, y \in W$, there exists an isomorphism ${ }^{y} L_{w}:{ }^{y} \mathscr{G} \rightarrow{ }^{w y} \mathscr{G}$ such that

$$
{ }^{y} L_{w}\left({ }^{y} \mathscr{G}_{\gamma}\right)={ }^{w y} \mathscr{G}_{w(\gamma)} \text { for } \quad \gamma \in{ }^{y} P
$$

where ${ }^{y} \mathscr{G}_{\gamma}$ and ${ }^{w y} \mathscr{G}_{w(\gamma)}$ are weight spaces (see Theorem 2.5.1; in the text, we only give ${ }^{y} L_{s_{i}}=L_{i}$ with a simple reflection $s_{i}$ ). This construction has been inspired by [FSS], [LSS $]$, etc. Let ${ }^{y} \Phi\left(\subset^{y} P\right)$ be the set of roots of ${ }^{y} \mathscr{G}$. Under the above identification, the lowest positive imaginary root $\delta^{\dagger} \in P^{\dagger}$ is identified with a root $\delta \in{ }^{y} \Phi$. Then ${ }^{y} \Phi$ is a union ${ }^{y} \Phi={ }^{y} \Phi_{r e} \cup^{y} \Phi_{i m}$ of ${ }^{y} \Phi_{i m}=\mathbf{Z} \delta \backslash\{0\}$ and ${ }^{y} \Phi_{r e}={ }^{y} \Phi \backslash \backslash^{y} \Phi_{i m}$. Using ${ }^{y} L_{w}$ 's, we get a uniform proof that $\operatorname{dim}^{y} \mathscr{G}_{\alpha}=1$ for $\alpha \in^{y} \Phi_{r e}$ (see Proposition 3.1.1).

We also define admissible Lie superalgebras ${ }^{y} \mathscr{G}^{b}$ 's with respect to $\left({ }^{y} \mathscr{E},{ }^{y} \Pi,{ }^{y} p\right)$ 's for $y \in W$, by using concrete defining relations, so that the following condition holds:

For each $y, w \in W$, there exists an isomorphism ${ }^{y} L_{w}^{b}:{ }^{y} \mathscr{G}^{b} \rightarrow{ }^{w y} \mathscr{G}^{b}$ satisfying the same equalities as in (1)

(see Steps 1-2 of Proof of Theorem 4.1.1, Proofs of Theorem 5.1.1 and Theorem 5.2.1; in the text, ${ }^{y} L_{w}^{b}$ is simply denoted by $L_{w}^{b}$ ). Using ${ }^{y} L_{w}^{b}$ 's, we can prove

$$
\operatorname{dim}{ }^{y} \mathscr{G}_{\alpha}^{b}=1 \text { for } \alpha \in{ }^{y} \Phi_{r e} .
$$

Likewise we can prove

$$
\operatorname{dim}{ }^{y} \mathscr{G}_{\gamma}^{b}=0 \text { for } \quad \gamma \in^{y} P \backslash\left({ }^{y} \Phi \cup\{0\}\right)
$$

(see Step 3 of Proof of Theorem 4.1.1).

Denote $\mathscr{G}^{b}$ by $\mathscr{G}^{b}$. Let $\mathscr{G}^{\natural}=\mathscr{G}^{\natural}(\mathscr{E}, \Pi, p)$ be a unique maximal Lie superalgebra among admissible ones with respect to the datum $(\mathscr{E}, \Pi, p)$ satisfying the properties (2) and (3) (Definition 3.1.2). By definition, the epimorphism $\Psi^{\natural b}=\Psi\left[\mathscr{G}^{\natural}, \mathscr{G}^{\natural}\right]$ exists, and we have ker $\Psi^{\natural b} \subset \oplus_{r \neq 0} \mathscr{G}_{r \delta}^{\natural}$. However, since none of the defining 
relations of $\mathscr{G}^{b}$ has weight $r \delta(r \neq 0), \Psi^{\natural b}$ is an isomorphism (see Step 4 of Proof of Theorem 4.1.1). Thus we get the defining relations of $\mathscr{G}^{\natural}$ (Theorem 4.1.1). Using the defining relations of $\mathscr{G}^{\natural}$, we get defining relations of $\mathscr{G}$ (Theorem 4.5.1, Theorem 5.1.1 and Theorem 5.2.1). We note that $\mathscr{G} \neq \mathscr{G}^{\natural}$ if and only if $\mathscr{G}$ is (ii), (v) or (vii) (Theorem 3.5.1).

0.4. The argument in 0.3 is more complicated than the one used, e.g. in [GK], in giving the defining relations for (non-super) symmetrizable $\mathrm{Kac}-\mathrm{Moody} \mathrm{Lie}$ algebras. A reason for this is that, although, in the case of the symmetrizable KacMoody Lie algebras, we can always use a key result (Proposition 1.2.2), the same is not true in our case. For example, when the affine Lie superalgebra $\mathscr{G}$ is (ii), (v) or (vii) (related to $\mathrm{A}(m, m)$ ), we cannot rely on the key result mentioned above, and the number of defining relations turns out to be infinite (Theorem 4.5.1).

0.5. We define the quantized universal enveloping superalgebra $U_{q}(\mathscr{G})$ abstractly in the same manner as in [Y1, Corollary 2.9.11]. (In the text, $U_{q}(\mathscr{G})$ is denoted as $U_{q}$ or $U_{q}(\mathscr{E}, \Pi, p)$.) A similar definition in the non-super case was introduced by Lusztig $[\mathrm{L} 1,3.1 .1]$. Let $U_{q}^{+}$be the subalgebra of $U_{q}(\mathscr{G})$ generated by $E_{i}$ 's. For our purpose, the following result is crucial:

$$
\text { If } X \in U_{q}^{+} \text {satisfies }\left[X, F_{i}\right]=0 \text { for any } i \text {, then } X=0
$$

(Proposition 6.5.1). By using (4), we get $q$-analogues of the defining relations of the affine Lie superalgebras $\mathscr{G}=\mathscr{G}(\mathscr{E}, \Pi, p)$ except for types (v) and (vii) (Proposition 6.7.1 and Proposition 8.4.2). Next we show that these $q$-analogues are indeed defining relations of $U_{q}(\mathscr{G})$ (Theorem 6.8.2 and Theorem 8.4.3). Unlike Lusztig [L1, Corollary 33.1.5] where a similar result is obtained in the non-super case (see also [Y1, Theorem 2.10.1]), our proof does not rely on the representation theory of $\mathscr{G}$ (see Proposition 6.8.1).

In $\S 7$, we give isomorphisms between the affine quantized universal enveloping superalgebras; these can be considered as $q$-analogues of ${ }^{y} L_{w}$ 's (see Proposition 7.4.1). In $\S 8$, by using these isomorphisms, and by using the same argument as in Beck [B], we consider super-versions of the Drinfeld generators (see [D3]) for $U_{q}\left(s l(m, n)^{(1)}\right)(m \neq n)$ and $U_{q}\left(\left(\mathbb{A}(m, m)^{(1)}\right)^{\mathscr{H}}\right)$, and get defining relations satisfied by these generators (see Theorem 8.5.1).

\section{§1. Preliminary}

1.1. In $\S 1$, we mainly refer to $[\mathrm{K} 1-2]$ and [VdL1-2].

We denote by $\mathbf{C}, \mathbb{Z}$, and $\mathbb{Z}_{+}$the field of complex numbers, the commutative ring of integers, and the semigroup of non-negative integers respectively. Put $\mathbb{C}^{\times}=$ 
$\mathbf{C} \backslash\{0\}$. Let $\mathbf{Z}_{2}$ be the cyclic group $\{\overline{0}, \overline{1}\}$ of order 2 . Let $V=V(\overline{0}) \oplus V(\overline{1})$ be a $\mathbf{Z}_{2}$-graded $\mathbf{C}$-linear space. If $X \in V(\bar{i})(i=0,1)$, then we say that $X$ is a homogeneous element of degree $i$ and write $p(X)=i ; p(X)$ is called the parity of $X$. A Lie superalgebra $\mathbf{g}$ is a $\mathbf{Z}_{2}$-graded space $\mathbf{g}=\mathbf{g}(\overline{0}) \oplus \mathbf{g}(\overline{1})$ equipped with a bilinear operation $[]:, \mathbf{g} \times \mathbf{g} \rightarrow \mathbf{g}$ such that

$$
\begin{aligned}
& {[X, Y]=-(-1)^{p(X) p(Y)}[Y, X] \in \mathbf{g}(\overline{p(X)+p(Y)}),} \\
& {[X,[Y, Z]]=[[X, Y], Z]+(-1)^{p(X)_{p}(Y)}[Y,[X, Z]]}
\end{aligned}
$$

for homogeneous elements $X, Y, Z$. A bilinear form $(\mid): \mathbf{g} \times \mathbf{g} \rightarrow \mathbf{C}$ satisfying $(X \mid Y)=(-1)^{p(X)_{p}(Y)}(Y \mid X)$ and $([X, Y] \mid Z)=(X \mid[Y, Z])$ for homogeneous elements $X, Y, Z$ is called an invariant form on $\mathrm{g}$.

For $X \in \mathbf{g}$, we define $\operatorname{ad}(X): \mathbf{g} \rightarrow \mathbf{g}$ by $\operatorname{ad}(X)(Y)=[X, Y](Y \in \mathbf{g})$. Following [VdL1-2], we define a Lie superalgebra $\hat{\mathbf{g}}=\mathbf{g} \otimes_{\mathbf{C}} \mathbf{C}\left[t, t^{-1}\right] \oplus \mathbf{C} \boldsymbol{c} \oplus \mathbf{C} d$ by

$$
\begin{aligned}
& {\left[X \otimes t^{m}+a_{1} c+b_{1} d, Y \otimes t^{n}+a_{2} c+b_{2} d\right]} \\
& =[X, Y] \otimes t^{m+n}+m \delta_{m+n, 0}(X \mid Y) c+b_{1} n Y \otimes t^{n}-b_{2} m X \otimes t^{m}
\end{aligned}
$$

where $\hat{\mathbf{g}}(0)=\mathbf{g}(0) \otimes_{\mathbf{C}} \mathbf{C}\left[t, t^{-1}\right] \oplus \mathbf{C} \boldsymbol{c} \oplus \mathbf{C} d$ and $\hat{\mathbf{g}}(1)=\mathbf{g}(1) \otimes_{\mathbf{C}} \mathbf{C}\left[t, t^{-1}\right]$.

Let $\tau: \mathbf{g} \rightarrow \mathbf{g}$ be an automorphism of $\mathbf{g}$ of finite order $r$. Put

$$
\mathbf{g}_{n}^{\tau}=\left\{X \in \mathbf{g} \mid \tau(X)=\left(\exp \frac{2 \pi n \sqrt{-1}}{r}\right) X\right\} \quad(0 \leq n<r) .
$$

Then $\mathbf{g}_{0}^{\tau}$ is a subalgebra of $\mathbf{g}$, and $\mathbf{g}_{i}^{\tau}(1 \leq i \leq r-1)$ are $\mathbf{g}_{0}^{\tau}$-modules. We define a subalgebra $L(\mathrm{~g}, \tau)$ of $\hat{\mathbf{g}}$ by

$$
L(\mathbf{g}, \tau)=\bigoplus_{n=0}^{r-1}\left(\bigoplus_{m \in \mathbf{Z}} \mathbf{g}_{n}^{\tau} \otimes t^{m r+n}\right) \oplus \mathbf{C} c \oplus \mathbf{C} d
$$

Obviously $L(\mathbf{g}, 1)=\hat{\mathbf{g}}$.

1.2. Here we give a definition of the (symmetrizable) Kac-Moody Lie superalgebra in an abstract manner similar to the one of the Kac-Moody Lie algebra given in $[\mathrm{K} 1, \S 1.3]$. Let $\mathscr{E}$ be a finite dimensional $\mathbf{C}$-vector space with a nondegenerate symmetric bilinear form ( , ). Let $\Pi=\left\{\alpha_{0}, \alpha_{1}, \ldots, \alpha_{n}\right\}$ be a linearly independent subset of $\mathscr{E}$. Put $\boldsymbol{P}=\mathbf{Z} \alpha_{0} \oplus \cdots \oplus \mathbf{Z} \alpha_{n}, \boldsymbol{P}_{+}=\mathbf{Z}_{+} \alpha_{0} \oplus \cdots \oplus \mathbf{Z}_{+} \alpha_{n}$, and $\boldsymbol{P}_{-}$ $=-P_{+}$. We call an element $\alpha_{i} \in \Pi$ a simple root, and $P$ the root lattice. Any function $p: \Pi \rightarrow \mathbf{Z}_{2}$ can be uniquely extended to the group homomorphism $p: P \rightarrow \mathbf{Z}_{2}$. Put $\mathscr{H}=\mathscr{E}^{*}$. We identify an element $\nu \in \mathscr{E}$ with $H_{\nu} \in \mathscr{H}$ satisfying $\mu\left(H_{\nu}\right)=(\mu, \nu)$ $(\mu \in \mathscr{E})$. A datum $(\mathscr{E}, \Pi, p)$ is a triple of $\mathscr{E}, \Pi$ and $p$ as above. For a datum $(\mathscr{E}, \Pi, p)$, we define a Lie superalgebra $\tilde{\mathscr{G}}=\tilde{\mathscr{G}}(\mathscr{E}, \Pi, p)$ by generators: 


$$
H \in \mathscr{H}, E_{i}, F_{i} \quad(0 \leq i \leq n),
$$

relations:

$$
\begin{gathered}
{\left[H, H^{\prime}\right]=0 \quad\left(H, H^{\prime} \in \mathscr{H}\right),} \\
{\left[H, E_{i}\right]=\alpha_{i}(H) E_{i}, \quad\left[H, F_{i}\right]=-\alpha_{i}(H) F_{i},} \\
{\left[E_{i}, F_{j}\right]=\delta_{i j} H_{\alpha_{i}},}
\end{gathered}
$$

and parities:

$$
p\left(E_{i}\right)=p\left(F_{i}\right)=p\left(\alpha_{i}\right), \quad p(H)=0(H \in \mathscr{H}) .
$$

The superalgebra $\tilde{\mathscr{G}}$ has a triangular decomposition:

$$
\tilde{\mathscr{G}}=\tilde{\mathscr{N}}^{+} \oplus \mathscr{H} \oplus \tilde{\mathscr{N}}^{-}
$$

where $\tilde{N}^{+}$(resp. $\tilde{\mathscr{N}}^{-}$) is the free superalgebra with generators $E_{i}$ (resp. $F_{i}$ ). See [K2] and [VdL1-2].

Definition 1.2.1. We say that an ideal $r^{\#}$ of $\tilde{\mathscr{G}}$ is admissible if $r^{\sharp} \cap \mathscr{H}=\{0\}$. We also say that the quotient $\mathscr{G}^{\#}=\mathscr{G}^{\#}(\mathscr{E}, \Pi, p)=\tilde{\mathscr{G}} / r^{\#}$ is admissible if $r^{\#}$ is an admissible ideal. For a fixed datum $(\mathscr{E}, \Pi, p)$, corresponding admissible Lie superalgebras form a partially ordered set $I(\mathscr{E}, \Pi, p)$ with a partial order $>$; for two elements $\mathscr{G}^{\#}=\tilde{\mathscr{G}} / r^{\#}$ and $\mathscr{G}^{\# \#}=\tilde{\mathscr{G}} / r^{\#}$ of $I(\mathscr{E}, \Pi, p)$, we write $\mathscr{G}^{\#}>\mathscr{G}^{\#}$ if $r^{\#} \subset r^{\#}$. Clearly, $\tilde{\mathscr{G}}$ is the unique top element of $I(\mathscr{E}, \Pi, p)$. We note that $\mathscr{G}^{\#}>\mathscr{G}^{\#}$ if and only if there is an epimorphism $\Psi\left[\mathscr{G}^{\#}, \mathscr{G}^{\# \#}\right]: \mathscr{G}^{\sharp \rightarrow} \mathscr{G}^{\# \#}\left(H, E_{i}, F_{i} \rightarrow H, E_{i}, F_{i}\right)$. We denote by $\mathscr{G}=\mathscr{G}(\mathscr{E}, \Pi, p)$ the unique botom element of $I(\mathscr{E}, \Pi, p)$.

For $\mathscr{G}^{\sharp}=\mathscr{G}^{\sharp}(\mathscr{E}, \Pi, p) \in I(\mathscr{E}, \Pi, p)$ and $\alpha \in \mathscr{E}$, let $\mathscr{G}_{\alpha}^{\#}=\{X \in \mathscr{G} \mid[H, X]=$ $\alpha(H) X(H \in \mathscr{H})\}$ and $\Phi\left[\mathscr{G}^{\sharp}\right]=\left\{\alpha \in \mathscr{E} \backslash\{0\} \mid \operatorname{dim} \mathscr{G}_{\alpha}^{\sharp} \neq 0\right\}$. The linear space $\mathscr{G}_{0}^{\#}=\mathscr{H}$ is called the Cartan subalgebra of $\mathscr{G}^{\#}$. Clearly, $\Phi\left[\mathscr{G}^{\#}\right] \subset P_{+} \cup P_{-} \backslash\{0\}$. We put $\Phi(\mathscr{E}, \Pi, p)=\Phi[\mathscr{G}]$. We note that $\mathscr{G}^{\#}>\mathscr{G}^{\#}$ implies $\Phi\left[\mathscr{G}^{\#}\right] \supset \Phi\left[\mathscr{G}^{\#}\right]$.

For a subset $B$ of $I(\mathscr{E}, \Pi, p)$, we define an admissible Lie superalgebra $\vee_{\mathscr{G} \sharp \in B} \mathscr{G} \notin I(\mathscr{E}, \Pi, p)$ by

$$
\vee_{\mathscr{G}^{\sharp} \in B} \mathscr{G}^{\sharp}=\tilde{\mathscr{G}} /\left(\cap_{\mathscr{G}^{\sharp} \in B} \operatorname{ker} \Psi\left[\tilde{\mathscr{G}}, \mathscr{G}^{\sharp}\right]\right) .
$$

Then we see that $\vee_{\mathscr{G}_{\sharp}^{\#} \in B} \mathscr{G}^{\#}>\mathscr{G}^{\# \#}$ for $\mathscr{G}^{\# \#} \in B$, and that $\Phi\left[\vee_{\mathscr{G}^{\#} \in B} \mathscr{G}^{\sharp}\right]=\cup_{\mathscr{G}_{\sharp} \in B} \Phi\left[\mathscr{G}^{\sharp}\right]$. We note that, for a fixed $\alpha \in \mathscr{E}$, if $\operatorname{dim} \mathscr{G}_{\alpha}^{\#}$ is independent of $\mathscr{G}^{\# \#} \in B$, then

$$
\operatorname{dim}\left(\vee_{\mathscr{G}^{\sharp} \in B} \mathscr{G}^{\sharp}\right)_{\alpha}=\operatorname{dim} \mathscr{G}_{\alpha}^{\# \#}
$$


for $\mathscr{G}^{\# \#} \in \boldsymbol{B}$.

For $\beta, \alpha \in \boldsymbol{P}_{+}$, we write $\beta<\alpha$ if $\alpha-\beta \in \boldsymbol{P}_{+} \backslash\{0\}$. By the same argument used in the proof of $[\mathrm{K} 1$, Proposition 9.11] (see also $[\mathrm{GK}]$ ), we have

Proposition 1.2.2. For a datum $(\mathscr{E}, \Pi, p)$, let $\rho \in \mathscr{E}$ be an element such that $\left(\rho, \alpha_{i}\right)=\left(\alpha_{i}, \alpha_{i}\right) / 2$ for any $\alpha_{i} \in \Pi$. Let $\mathscr{G}^{\sharp} \in I(\mathscr{E}, \Pi, p)$. Then, if $\alpha \in P_{+}$is an element such that $(\alpha, \alpha) \neq 2(\rho, \alpha)$ and such that $\operatorname{dim} \mathscr{G}_{\beta}^{*}=\operatorname{dim} \mathscr{G}_{\beta}$ for any $\beta \in \boldsymbol{P}_{+}$ with $\beta<\alpha$, then $\operatorname{dim} \mathscr{G}_{\alpha}^{\#}=\operatorname{dim} \mathscr{G}_{\alpha}$.

Lemma 1.2.3. Let $\mathscr{G}^{\#} \in I(\mathscr{E}, \Pi, p)$. Let $\alpha_{i} \in \Pi$. We have the following.

$$
\operatorname{dim} \mathscr{G}_{m \alpha_{i}}^{\#}= \begin{cases}1 & \text { if } m=1, \\ 1 & \text { if } m=2,\left(\alpha_{i}, \alpha_{i}\right) \neq 0 \text { and } p\left(\alpha_{i}\right)=1 \\ 0 & \text { if } m=2 \text { and } p\left(\alpha_{i}\right)=0 \\ 0 & \text { if } m \geq 3\end{cases}
$$

(ii) If $p\left(\alpha_{i}\right)=1$ and $\left(\alpha_{i}, \alpha_{i}\right)=0$, then $\operatorname{dim} \mathscr{G}_{2 \alpha_{i}}^{\#}=0$ if and only if $\left[E_{i}, E_{i}\right]=0$.

(iii) The statements of (i) and (ii) with $-\alpha_{i}$ instead of $\alpha_{i}$ also hold.

(iv) If $\beta \in P \backslash\left(P_{+} \cup P_{-}\right)$, then $\operatorname{dim} \mathscr{G}_{\beta}^{\sharp}=0$.

Proof. (i) Since $\mathscr{G}_{m \alpha_{i}}^{\#}$ is spanned by the element $\left[\ldots\left[E_{i}, E_{l}\right], \ldots, E_{i}\right]\left(E_{i}\right.$ appears $m$-times), it is clear that $\mathscr{G}_{m \alpha_{i}}^{\sharp}=0$ if $m \geq 3$, or if $m=2$ with $p\left(\alpha_{i}\right)=0$. Since $\left[E_{i}, F_{i}\right]=H_{\alpha_{i}} \neq 0, \operatorname{dim} \mathscr{G}_{\alpha_{i}}^{\#}=1$. Similarly $\operatorname{dim} \mathscr{G}_{2 \alpha_{i}}^{\#}=1$ if $\left(\alpha_{i}, \alpha_{i}\right) \neq 0$ and $p\left(\alpha_{i}\right)=1$.
(ii), (iii) and (iv) are easy.
Q. E. D.

Lemma 1.2.4. Let $\mathscr{G}^{\#} \in I(\mathscr{E}, \Pi, p)$. Let $a_{i} \in \mathbf{C}^{\times}(1 \leq i \leq m=|\Pi|)$. Then there exists a unique automorphism $I\left(a_{1}, \ldots, a_{m}\right): \mathscr{G}^{\#}(\mathscr{E}, \Pi, p) \rightarrow \mathscr{G}^{\#}(\mathscr{E}, \Pi, p)$ such that

$$
H, E_{i}, F_{i} \rightarrow H, a_{i} E_{i}, a_{i}^{-1} F_{i}
$$

Moreover a homomorphism $\phi: \mathscr{G}^{\#} \rightarrow \mathscr{G}^{\#}$ satisfies $\phi_{\mid \mathscr{H}}=1_{\mathscr{H}}$ if and only if $\phi=I\left(a_{1}, \ldots\right.$, $\left.a_{m}\right)$ for some $a_{i} \in \mathbf{C}^{\times}(1 \leq i \leq m)$.

Proof. Let $r^{\sharp}=\operatorname{ker} \Psi\left[\tilde{\mathscr{G}}, \mathscr{G}^{\sharp}\right]$. Then the first statement follows from: $r^{\sharp} \cap \mathscr{H}$ $=\{0\},\left[\mathscr{H}, r^{\sharp}\right] \subset r^{\sharp}$ and $r^{\#}=\oplus_{\alpha \in P \backslash\{0\}}\left(r^{\sharp} \cap \tilde{\mathscr{G}}_{\alpha}\right)$. The second statement follows from: $\operatorname{dim} \mathscr{G}_{ \pm \alpha_{i}}^{\#}=1$ (see Lemma 1.2.3) and $\left[\phi\left(E_{i}\right), \phi\left(F_{i}\right)\right]=H_{\alpha_{i}} \neq 0$. Q. E. D.

Definition 1.2.5. Assume that $\mathscr{G}^{\#}=\mathscr{G}^{\#}(\mathscr{E}, \Pi, p)$ and $\mathscr{G}^{\sharp}=\mathscr{G}^{\sharp}\left(\mathscr{E}^{\prime}, \Pi^{\prime}, p^{\prime}\right)$ are admissible. Let $\phi: \mathscr{G}^{\sharp} \rightarrow \mathscr{G}^{\sharp \prime}$ and $\varphi: \mathscr{G}^{\sharp} \rightarrow \mathscr{G}^{\sharp \prime}$ be homomorphisms. We write $\phi \equiv \varphi$ if

$$
\varphi=\phi \circ I\left(a_{1}, \ldots, a_{m}\right) \text { for some } a_{i} \in \mathbf{C}^{\times}
$$


Then $\equiv$ is an equivalence relation. If $\phi$ and $\varphi$ are isomorphisms such that $\phi(\mathscr{H})$ $=\varphi(\mathscr{H})=\mathscr{H}$, then $\phi \equiv \varphi$ if and only if

$$
\varphi=I\left(b_{1}, \ldots, b_{m}\right) \circ \phi \quad \text { for some } b_{i} \in \mathbb{C}^{\times}
$$

1.3. Here we introduce the notion of the Dynkin diagram associated with a datum $(\mathscr{E}, \Pi, p)$. We need the three types of vertices:

$$
\underset{\text { (white) }}{\bigcirc}, \underset{\text { (gray) }}{\otimes} \text { (black) }
$$

We call them white, gray and black vertices respectively. For the $i$-th simple root $\alpha_{i}$, the corresponding $i$-th vertex is determined by the following rule:

$$
\begin{array}{ll}
\text { white } & \text { if }\left(\alpha_{i}, \alpha_{i}\right) \neq 0 \text { and } p\left(\alpha_{i}\right)=0, \\
\text { gray } & \text { if }\left(\alpha_{i}, \alpha_{i}\right)=0 \text { and } p\left(\alpha_{i}\right)=1, \\
\text { black if }\left(\alpha_{i}, \alpha_{i}\right) \neq 0 \text { and } p\left(\alpha_{i}\right)=1 .
\end{array}
$$

The vertex $\cdot$ can be any one of these three types of vertices. The vertex $\times$ can be white or gray. The vertex - can be white or black.

Concerning the edge between the $i$-th and the $j$-th vertices, we write:

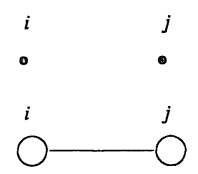

$$
\begin{array}{r}
\text { if }\left(\alpha_{i}, \alpha_{j}\right)=0, \\
\text { if }\left(\alpha_{i}, \alpha_{i}\right)=\left(\alpha_{j}, \alpha_{j}\right)=-2\left(\alpha_{i}, \alpha_{j}\right) \neq 0, \\
\text { if }\left(\alpha_{i}, \alpha_{i}\right)=\left(\alpha_{j}, \alpha_{j}\right)=-\left(\alpha_{i}, \alpha_{j}\right) \neq 0,
\end{array}
$$
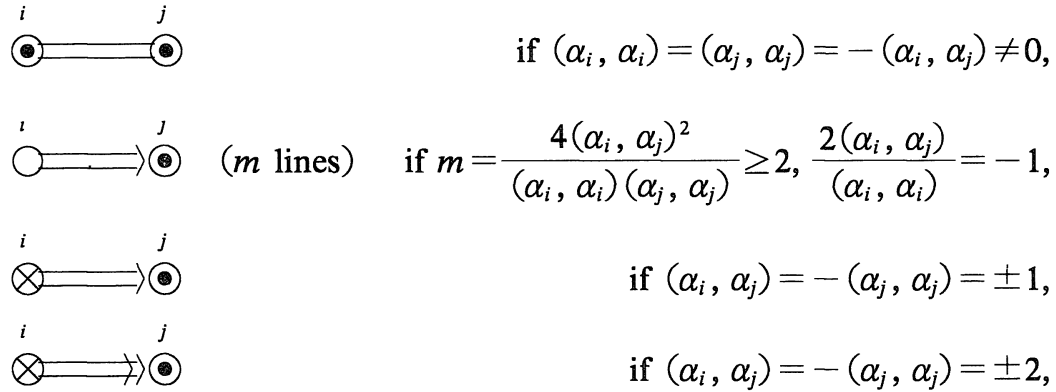

$$
\begin{aligned}
& \text { if }\left(\alpha_{i}, \alpha_{j}\right)=-\left(\alpha_{j}, \alpha_{j}\right)= \pm 1, \\
& \text { if }\left(\alpha_{i}, \alpha_{j}\right)=-\left(\alpha_{j}, \alpha_{j}\right)= \pm 2,
\end{aligned}
$$

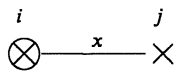

$$
\text { if } x=\left(\alpha_{i}, \alpha_{j}\right) \in \mathbf{C}^{\times} \text {, and }\left(\alpha_{j}, \alpha_{j}\right)=-2 x \text { if }\left(\alpha_{j}, \alpha_{j}\right) \neq 0 .
$$

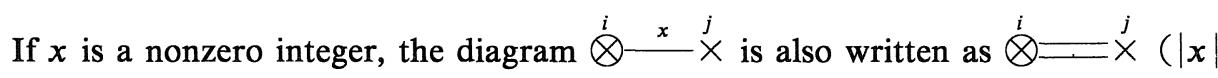
lines). Moreover $\stackrel{i}{\otimes}=\stackrel{j}{O}^{j}$ is also written as $\stackrel{i}{\otimes}^{=} \stackrel{\rho}{O}^{j}$. The diagram $\times$ :..... $\times$ can be $\bigcirc \quad \bigcirc$ or $\otimes=\otimes$. 
An edge between the $i$-th and the $j$-th vertices is called an $i-j$ edge.

Assume $\left(\alpha_{j}, \alpha_{j}\right)=0,\left(\alpha_{i}, \alpha_{j}\right) \in \mathbf{Z} \backslash\{0\}$ and $\left(\alpha_{j}, \alpha_{k}\right) \in \mathbf{Z} \backslash\{0\}$. If we write a short line crossing the $i-j$ edge and a short line crossing the $j-k$ edge, it means that both $\left(\alpha_{i}, \alpha_{j}\right)$ and $\left(\alpha_{j}, \alpha_{k}\right)$ are nonzero integers of the same sign. Otherwise the signs of $\left(\alpha_{i}, \alpha_{j}\right)$ and $\left(\alpha_{j}, \alpha_{k}\right)$ are different. For example, $\stackrel{i}{\times} \longrightarrow^{j}={ }^{k} \times$ implies that $2\left(\alpha_{i}\right.$,

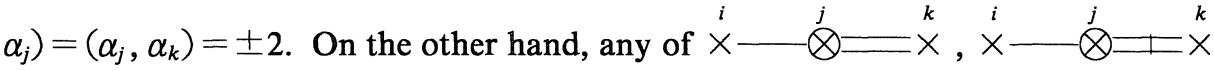
and $\stackrel{i}{\times}+\stackrel{j}{\otimes}^{-}=\stackrel{k}{\times}$ implies that $-2\left(\alpha_{i}, \alpha_{j}\right)=\left(\alpha_{j}, \alpha_{k}\right)= \pm 2$.

Although we occasionally write short lines crossing the $i-j$ and/or $j-k$ edges even in the case $\left(\alpha_{j}, \alpha_{j}\right) \neq 0$, these lines should be disregarded.

It is true that, for some datum, the corresponding Dynkin diagram is not defined by the above rule alone. However, for any datum discussed in this paper, its Dynkin diagram is defined by the above rule. If the Dynkin diagrams of two data $(\mathscr{E}, \Pi, p)$ and $\left(\mathscr{E}^{\prime}, \Pi^{\prime}, p^{\prime}\right)$ are well defined by the above rule and the resulting diagrams are the same and connected, then there is $c \in \mathbf{C}^{\times}$and a parity preserving bijective map $f_{\Pi}: \Pi \rightarrow \Pi^{\prime}$ such that $\left(f_{\Pi}(\alpha), f_{\Pi}(\beta)\right)=c(\alpha, \beta)$ for $\alpha, \beta \in \Pi$. Moreover, if $\operatorname{dim} \mathscr{E} \leq \operatorname{dim} \mathscr{E}^{\prime}, f_{\Pi}$ is uniquely extended to a linear injective map $f_{\mathscr{E}}: \mathscr{E}$ $\rightarrow \mathscr{E}^{\prime}$ such that $\left(f_{\mathscr{E}}(\mu), f_{\mathscr{E}}(\nu)\right)=c(\mu, \nu)$ for $\mu, \nu \in \mathscr{E}$, and $f_{\mathscr{E}}$ induces a unique monomorphism $f_{\mathscr{G}}: \mathscr{G}(\mathscr{E}, \Pi, p) \rightarrow \mathscr{G}\left(\mathscr{E}^{\prime}, \Pi^{\prime}, p^{\prime}\right)$ such that $f_{\mathscr{G}}\left(H_{\nu}\right)=c^{-1} H_{f_{\mathscr{E}}(\nu)}$.

Caution. Two Kac-Moody Lie superalgebras corresponding to distinct Dynkin diagrams may be isomorphic.

1.4. Here we list several data $\left(\mathscr{E}, \Pi=\left\{\alpha_{0}, \ldots, \alpha_{n}\right\}, p\right)$ with their Dynkin diagrams.

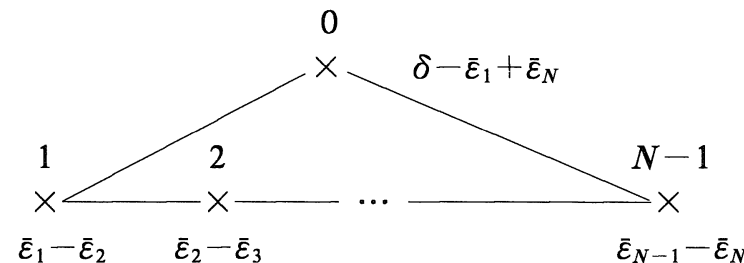

$(N=1)$

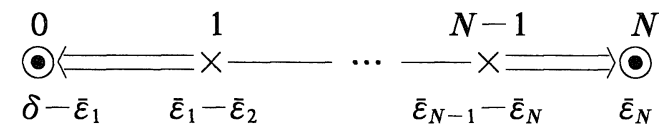

$$
(N=1)
$$
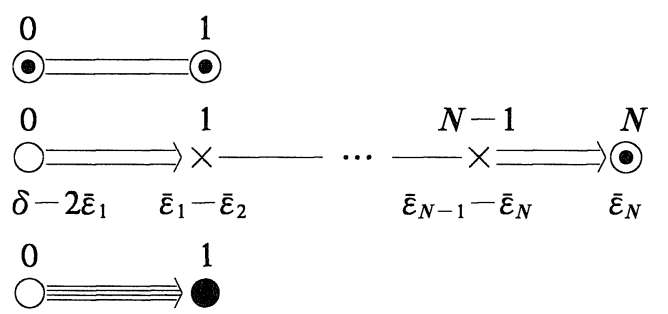

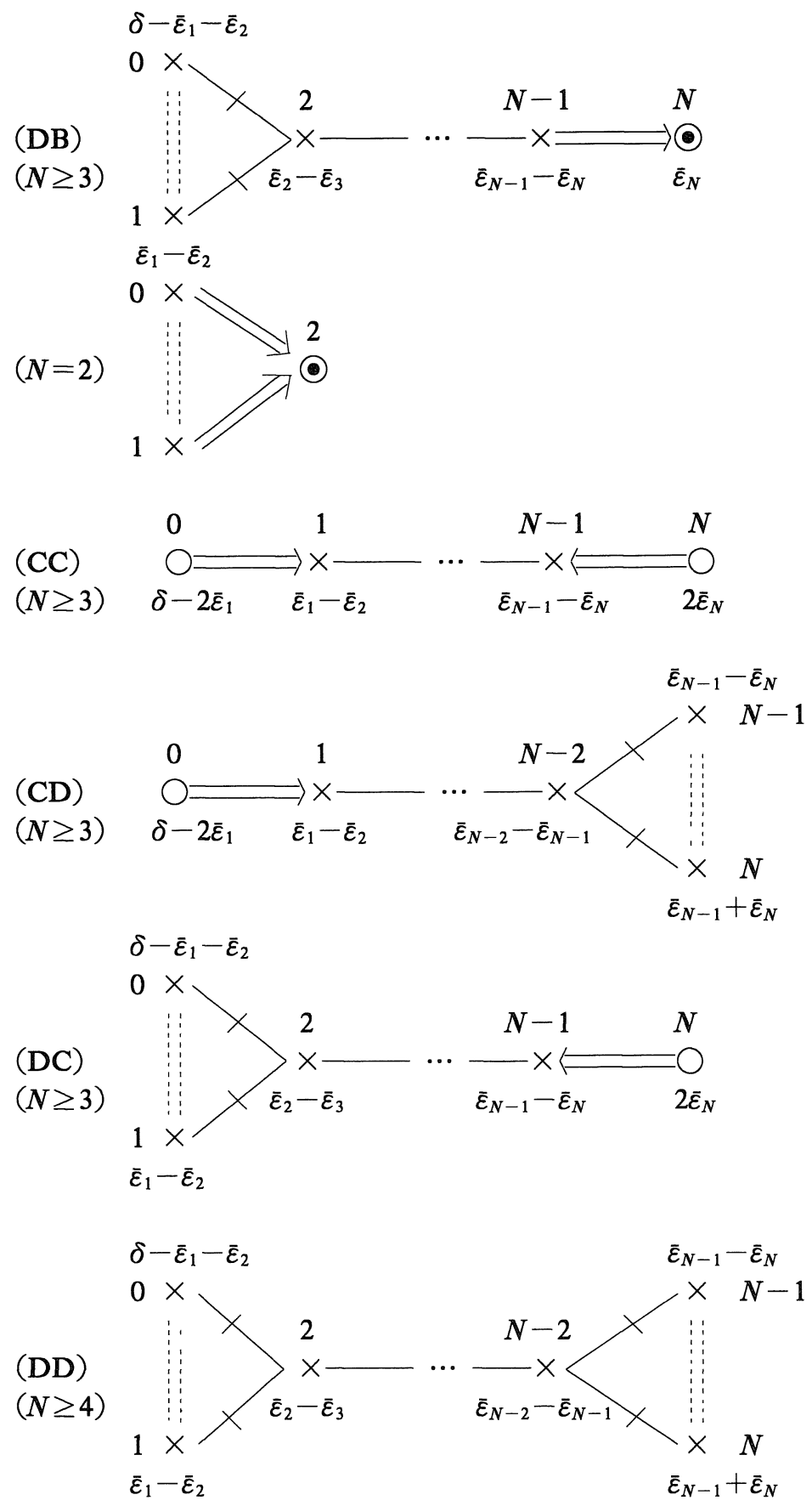


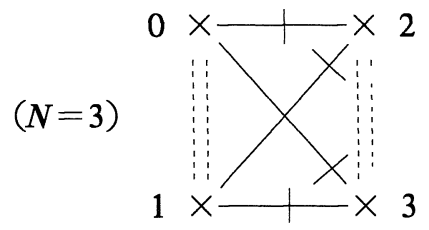

In the above list, we have given a name (XY) for each of the above Dynkin diagrams where $\mathbf{X}, \mathbf{Y}=\mathbf{A}, \mathbf{B}, \mathbf{C}$ or $\mathbf{D}$. We fix a datum $\left(\mathscr{E}, \Pi=\left\{\alpha_{0}, \ldots, \alpha_{n}\right\}, p\right)$ whose Dynkin diagram is (XY) as follows. The positive integer $n$ is defined by:

$$
n= \begin{cases}N-1 & \text { if }(\mathrm{XY}) \text { is }(\mathrm{AA}) \\ N & \text { otherwise }\end{cases}
$$

The $i$-th vertex corresponds to the $i$-th root $\alpha_{i}$. Let $\mathscr{E}^{\text {ex }}$ be an $(N+2)$-dimensional C-linear space with a nondegenerate symmetric form $($,$) and a basis \left\{\bar{\varepsilon}_{1}, \ldots, \bar{\varepsilon}_{N}\right.$, $\left.\delta, \Lambda_{0}\right\}$ such that

$$
\left(\bar{\varepsilon}_{i}, \bar{\varepsilon}_{j}\right)=\delta_{i j} \bar{d}_{i}\left(\bar{d}_{i}= \pm 1\right),\left(\bar{\varepsilon}_{i}, \delta\right)=(\delta, \delta)=\left(\Lambda_{0}, \Lambda_{0}\right)=0,\left(\delta, \Lambda_{0}\right)=1
$$

The subspace $\mathscr{E}$ of $\mathscr{E}^{e x}$ is defined by

$$
\mathscr{E}= \begin{cases}\left\{x \in \mathscr{E}^{e x} \mid(x, \theta)=0\right\} & \text { if }(\mathrm{XY})=(\mathrm{AA}) \text { and } \sum_{i=1}^{N} \bar{d}_{i} \neq 0, \\ \mathscr{E}^{e x} \text { if }(\mathrm{XY}) \neq(\mathrm{AA}) \text { or } & \text { if }(\mathrm{XY})=(\mathrm{AA}) \text { and } \sum_{i=1}^{N} \bar{d}_{i}=0\end{cases}
$$

where $\theta=\sum_{i=1}^{N} \bar{d}_{i} \bar{\varepsilon}_{i}$. Clearly (, ) induces a nondegenerate symmetric form on $\mathscr{E}$. For a fixed $N$, we denote by $\operatorname{ABCD}(N)$ the set of the above mentioned data satisfying the conditions (a) $\operatorname{dim} \mathscr{E}^{e x}=N+2$ and (b) there is a simple root whose parity $=\overline{1}$. For $(\mathscr{E}, \Pi, p) \in \operatorname{ABCD}(N)$, we call $\bar{\varepsilon}_{1}, \ldots, \bar{\varepsilon}_{N}, \delta, \Lambda_{0}$ the fundamental elements of $(\mathscr{E}, \Pi, p)$.

Definition 1.4.1. The Kac-Moody Lie superalgebra $\mathscr{G}(\mathscr{E}, \Pi, p)$ for some $(\mathscr{E}$, $\Pi, p) \in \operatorname{ABCD}(N)$ and some $N$ is called of affine ABCD-type.

1.5. Keep the notation in 1.4. In the table below, if we write $(X Y)_{i}$, it means

$$
i= \begin{cases}\sum_{i=1}^{N} p\left(\alpha_{i}\right)(\bmod 2) & \text { if }(\mathrm{XY}) \text { is }(\mathrm{BB}),(\mathrm{CB}) \text { or }(\mathrm{DB}), \\ \sum_{i=1}^{N} p\left(\alpha_{i}\right)(\bmod 2) & \text { otherwise. }\end{cases}
$$

The notation $(\mathrm{AA})^{g}$ (resp. $(\mathrm{AA})^{b}$ ) means that $(\mathrm{XY})$ is (AA) and $\sum_{i=1}^{N} \bar{d}_{i} \neq 0$ (resp. $\sum_{i=1}^{N} \bar{d}_{i}=0$ ). 
For a finite dimensional Kac-Moody Lie superalgebra $\mathbf{g}$ and an automorphism $\tau$ of $\mathbf{g}$ of order $k, \mathbf{g}^{(k)}$ stands for $L(\mathbf{g}, \tau)$ (see 1.1). In the table below, we describe $\mathrm{g}^{(k)}$ which is isomorphic to $\mathscr{G}(\mathscr{E}, \Pi, p)$ for some $(\mathscr{E}, \Pi, p) \in \operatorname{ABCD}(N)$. For the definition of $\tau$ in each case described in Table 1.5.1, see Table 4 of [VdL2]. In Table 1.5.1, we use the notation of $\mathrm{g}$ given in $[\mathrm{K} 2,2.1 .1-2]$.

Table 1.5. 1

\begin{tabular}{|c|c|}
\hline $\mathscr{G}(\mathscr{E}, \Pi, p)$ & Dynkin diagram \\
\hline $\mathrm{A}(m-1, N-m-1)^{(1)}(N \neq 2 m)$ & $(\mathrm{AA})^{g}$ \\
\hline$\left(\mathrm{A}\left(\frac{N}{2}-1, \frac{N}{2}-1\right)^{(1)}\right)^{\mathscr{H}}$ (see below) & $(\mathrm{AA})^{b}$ \\
\hline $\mathrm{B}(N-m, m)^{(1)}$ & $(\mathrm{DB})_{0},(\mathrm{CB})_{1}$ \\
\hline $\mathrm{A}(2 m-1,2 N-2 m)^{(2)}$ & $(\mathrm{DB})_{1},(\mathrm{CB})_{0}$ \\
\hline $\mathrm{C}(N)^{(1)}, \mathrm{D}(m, N-m)^{(1)}$ & $(\mathrm{CC})_{0},(\mathrm{CD})_{1},(\mathrm{DD})_{0},(\mathrm{DC})_{1}$ \\
\hline $\mathrm{A}(2 m-1,2 N-2 m-1)^{(2)}$ & $(\mathrm{CC})_{1},(\mathrm{CD})_{0},(\mathrm{DD})_{1},(\mathrm{DC})_{0}$ \\
\hline $\mathrm{C}(N+1)^{(2)}, \mathrm{D}(m, N+1-m)^{(2)}$ & $(\mathrm{BB})_{0}$ \\
\hline $\mathrm{A}(2 m, 2 N-2 m)^{(4)}$ & $(\mathrm{BB})_{1}$ \\
\hline
\end{tabular}

Since different notation for $g$ is used in $[\mathrm{K} 2,2.1 .1-2]$, we shall add some comments for the notation. We first note that $s l(m, r)=\mathrm{A}(m-1, r-1)(m \neq r)$, $s l(m, m) / \mathbf{C I}_{2 m}=\mathrm{A}(m-1, m-1), \operatorname{osp}(2 m+1,2 r)=\mathrm{B}(m, r), \operatorname{osp}(2,2 r-1)=$ $\mathrm{C}(r), \operatorname{osp}(2 m, 2 r)=\mathrm{D}(m, r)$ where $I_{2 m}$ denotes the $2 m \times 2 m$ unit matrix. We note that $\mathbf{A}(m-1, m-1)$ and $s l(m, m)$ are not Kac-Moody Lie superalgebras since their simple roots are linearly dependent and that $g l(m, m)$ is a Kac-Moody Lie superalgebra. The algebra $\left(\mathbf{A}(m-1, m-1)^{(1)}\right)^{\mathscr{H}}$ appearing in Table 1.5.1 is given as follows. Let $\left(s l(m, m)^{(1)}\right)^{\mathscr{H}}$ be a subalgebra $s l(m, m)^{(1)} \oplus \mathbb{C} E_{11}$ of $g l(m, m)^{(1)}$ where $E_{11}$ denotes the matrix having 1 in the $(1,1)$ position and 0 elsewhere. Then $\left(\mathbf{A}(m-1, m-1)^{(1)}\right)^{\mathscr{H}}$ is defined as a quotient $\left(s l(m, m)^{(1)}\right)^{\mathscr{H}} /\left(\oplus_{k \neq 0} \mathbb{C I}_{2 m} \otimes t^{k}\right)$. We note that $\mathbf{A}(m-1, m-1)^{(1)}$ is not a Kac-Moody Lie superalgebra since its simple roots are linearly dependent.

1.6. Let $D(1,2 ; x), F(4)$ and $G(3)$ be the notation used in $[\mathbb{K} 2]$, where these denote finite dimensional simple Lie superalgebras. Then infinite dimensional Lie superalgebras $\mathbb{D}(1,2 ; x)^{(1)}=\mathbb{L}\left(\mathbb{D}(1,2 ; x)^{(1)}, 1\right), F(4)^{(1)}=L(F(4), 1)$ and $G(3)^{(1)}$ $=L(\mathrm{G}(3), 1)$ are isomorphic to some symmetrizable Kac-Moody Lie superalgebras. We shall give the data and the Dynkin diagrams of $\mathrm{D}(1,2 ; \boldsymbol{x})^{(1)}$ (resp. $F(4)^{(1)}$, resp. $G(3)^{(1)}$ ) in 5.1 (resp. 5.2, resp. 5.3).

Definition 1.6.1. We say that a Lie superalgebra $\mathscr{G}$ is an affine Lie superalgebra if it is one of the following types: affine $\mathrm{ABCD}$ type, $\mathrm{D}(1,2 ; x)^{(1)}$, $\mathrm{F}(4)^{(1)}$ and $\mathrm{G}(3)^{(1)}$. 
The reason is due to the following theorem proved by Van de Laur [VdL1-2].

Theorem 1.6.2([VdL1-2]). Infinite dimensional symetrizable Kac-Moody Lie superalgebras of finite growth are exactly the affine Lie superalgebras, in the above sense.

\section{§2. Isomorphisms Associated with Affine Weyl Groups}

2.1. In $\S 2$, we introduce a family $\left\{L_{i}\right\}$ of isomorphisms between $\mathscr{G}(\mathscr{E}, \Pi, p)$ 's of affine ABCD type (see Theorem 2.5.1). The isomorphisms $L_{i}: \mathscr{G}(\mathscr{E}, \Pi, p) \rightarrow \mathscr{G}\left({ }^{s_{i}} \mathscr{E}\right.$, $\left.{ }^{s_{i}} \Pi,{ }^{s_{i}} p\right)$ are defined for the following parity preserving isometries $s_{i}: \mathscr{E}^{s_{i}} \mathscr{E}$ :

(a) reflections $s_{i}$ for non-null simple roots $\alpha_{i}$,

(b) super-reflections $s_{i}$ for null simple roots $\alpha_{i}$,

(c) diagram automorphisms $s_{i}$

(see Propositions 2.2.6-8 and Definition 2.2.4). If $L_{l}$ is defined for a superreflection $s_{i}$, then $(\mathscr{E}, \Pi, p)$ and $\left({ }^{s} \mathscr{E},{ }^{s_{i}} \Pi,{ }^{s_{i}} p\right)$ are not the same.

Remark 2.1.1. The super-reflections are known (see [FSS], [LS], [LSS], $[\mathbf{S}])$. Let $\mathbf{g}$ be a simple Lie superalgebra of type A-G. V. V. Serganova proved that, if $(\mathscr{E}, \Pi, p)$ and $\left(\mathscr{E}^{\prime}, \Pi^{\prime}, p^{\prime}\right)$ are two data such that $\mathscr{G}(\mathscr{E}, \Pi, p)$ and $\mathscr{G}\left(\mathscr{E}^{\prime}, \Pi^{\prime}, p^{\prime}\right)$ are isomorphic to $\mathrm{g}$, then there exist finite data $\left(\mathscr{E}_{k}, \Pi_{k}, p_{k}\right)(1 \leq k \leq r)$ such that $\left(\mathscr{E}_{1}, \Pi_{1}, p_{1}\right)=(\mathscr{E}, \Pi, p),\left(\mathscr{E}_{r}, \Pi_{r}, p_{r}\right)=\left(\mathscr{E}^{\prime}, \Pi^{\prime}, p^{\prime}\right)$ and $\left(\mathscr{E}_{k}, \Pi_{k}, p_{k}\right)=$ $\left({ }^{s_{i(k-1)}}\left(\mathscr{E}_{k-1}\right),{ }^{s_{i(k-1)}}\left(\Pi_{k-1}\right),{ }^{s_{i(k-1)}}\left(p_{k-1}\right)\right)$ where $s_{i(k-1)}$ is a reflection or a superreflection (see [LSS, Appendix]).

2.2. The formulas in the following lemma are useful.

Lemma 2.2.1. Let $\left(\mathscr{E}, \Pi=\left\{\alpha_{1}, \ldots, \alpha_{n}\right\}, p\right)$ be a datum. The following equalities hold for $\tilde{\mathscr{G}}=\tilde{\mathscr{G}}(\mathscr{E}, \Pi, p)$.

$$
\begin{aligned}
& {\left[\left[E_{j}, E_{i}\right], F_{i}\right]=-\left(\alpha_{i}, \alpha_{j}\right)\left(1-\delta_{i j} p\left(\alpha_{i}\right)\right) E_{j},} \\
& {\left[E_{i},\left[F_{j}, F_{i}\right]\right]=\left(-\delta_{i j}+(-1)^{p\left(\alpha_{i}\right) p\left(\alpha_{j}\right)}\right)\left(\alpha_{i}, \alpha_{j}\right) F_{j},} \\
& {\left[\left[E_{j}, E_{i}\right],\left[F_{j}, F_{i}\right]\right]=(-1)^{p\left(\alpha_{i}\right) p\left(\alpha_{j}\right)}\left(\alpha_{i}, \alpha_{j}\right)\left(1-\delta_{i j} p\left(\alpha_{i}\right)\right) H_{\alpha_{i}+\alpha_{j}},} \\
& {\left[\left[\left[E_{i}, E_{j}\right], E_{k}\right], F_{j}\right]=0 \text { if }\left[E_{i}, E_{k}\right]=0 .}
\end{aligned}
$$

(ii) Let $\alpha_{i} \in \Pi$ satisfy $\left(\alpha_{i}, \alpha_{t}\right) \neq 0$. Let $a_{i j}=\frac{2\left(\alpha_{i}, \alpha_{i}\right)}{\left(\alpha_{i}, \alpha_{i}\right)}$ and $d=\frac{\left(\alpha_{i}, \alpha_{t}\right)}{2}$. Put 


$$
\left\langle k ;-a_{i j}\right\rangle=\left\{\begin{array}{cl}
k\left(-a_{i j}-k+1\right) \cdot d & \text { if } p\left(\alpha_{i}\right)=0 \\
k \cdot d & \text { if } p\left(\alpha_{i}\right)=1 \text { and } k \text { is even } \\
\left(-a_{i j}-k+1\right) \cdot d & \text { if } p\left(\alpha_{i}\right)=1 \text { and } k \text { is odd }
\end{array}\right.
$$

Then we have

$$
\begin{aligned}
& {\left[E_{i}, \operatorname{ad}\left(F_{i}\right)^{k}\left(F_{j}\right)\right]=(-1)^{(k-1) p\left(\alpha_{i}\right)}\left\langle k ;-a_{i j}\right\rangle \operatorname{ad}\left(F_{i}\right)^{k-1}\left(F_{j}\right),} \\
& {\left[F_{i}, \operatorname{ad}\left(E_{i}\right)^{k}\left(E_{j}\right)\right]=(-1)^{k p\left(\alpha_{j}\right)}\left\langle k ;-a_{i j}\right\rangle \operatorname{ad}\left(E_{i}\right)^{k-1}\left(E_{j}\right) .}
\end{aligned}
$$

(iii) Let $W, X, Y \in \tilde{\mathscr{G}}$.

$$
[X,[X, Y]]=0 \text { if } p(X)=1 \text { and }[X, X]=0 .
$$

$[[X, Y],[X, Y]]=0$ if $p(X)=1$ and $[X, X]=[[X, Y], Y]=0$.

$[[[X, Y], W], Y]=0$ if $p(Y)=0$ and $[[X, Y], Y]=[[W, Y], Y]=[X, W]=0$.

Proof. These can be checked directly. For example, the third equality of (iii) holds since $2[[[X, Y], W], Y]=[[[X, Y], Y], W]-[X,[Y,[Y, W]]]$. (We note that the equalities in (iii) hold in an arbitrary superalgebra.) Q. E. D.

Lemma 2.2.2. Let $\mathscr{N}^{+}=\Psi[\tilde{\mathscr{G}}, \mathscr{G}]\left(\tilde{\mathscr{N}}^{+}\right)$and $\mathscr{N}^{-}=\Psi[\tilde{\mathscr{G}}, \mathscr{G}]\left(\tilde{\mathscr{N}}^{-}\right)$. Let $X \in$ $\mathscr{N}^{+}\left(\right.$resp. $\left.Y \in \mathscr{N}^{-}\right)$be such that $\left[X, F_{k}\right]=0$ (resp. $\left.\left[E_{k}, Y\right]=0\right)$ for any $k$. Then $X$ $=0($ resp. $Y=0)$ in $\mathscr{G}$.

Proof. We may assume that $X$ is in some root space. Let $r_{+}(X)$ be the ideal of $\mathscr{N}^{+}$generated by $X$. Then $r_{+}(X)$ is an ideal of $\mathscr{G}$ such that $r_{+}(X) \cap \mathscr{H}=0$. Hence $X=0$.

Q. E. D.

As an immediate consequence of Lemma 2.2.1 and Lemma 2.2.2, we have

Lemma 2.2.3. Keep the above nototion. The following equalities hold for $\mathscr{G}(\mathscr{E}$, $\Pi, p)$.

(i) Let $\alpha_{i} \in \Pi$ satisfy $\left(\alpha_{i}, \alpha_{i}\right) \neq 0$. Assume that $-a_{i j} \in 2 \mathbb{Z}_{+}$if $p\left(\alpha_{i}\right)=1$ and $-a_{i j} \in \mathbb{Z}_{+}$. Then

$$
\operatorname{ad}\left(E_{i}\right)^{1-a_{i j}}\left(E_{j}\right)=\operatorname{ad}\left(F_{i}\right)^{1-a_{i j}}\left(F_{j}\right)=0 .
$$

(ii) If $\left(\alpha_{i}, \alpha_{i}\right)=0$, then

$$
\left[E_{i}, E_{i}\right]=\left[F_{i}, F_{i}\right]=0 .
$$


Definition 2.2.4. (i) Let $(\mathscr{E}, \Pi, p)$ and $\left(\mathscr{E}^{\prime}, \Pi^{\prime}, p^{\prime}\right)$ be data. For a bijective linear map $f: \mathscr{E} \rightarrow \mathscr{E}^{\prime}$, we say that $f$ is a parity preserving isometry if $f$ satisfies the following conditions:

(a) $(f(\gamma), f(\mu))=(\gamma, \mu)(\gamma, \mu \in \mathscr{E})$,

(b) $|\Pi|=\left|\Pi^{\prime}\right|$, and, $f(\Pi) \subset P^{\prime}$ where $P^{\prime}$ is the root lattice of $\left(\mathscr{E}^{\prime}, \Pi^{\prime}, p^{\prime}\right)$,

(c) $p\left(\alpha_{i}\right)=p^{\prime}\left(f\left(\alpha_{i}\right)\right)$ for any $\alpha_{i} \in \Pi$.

(ii) We say that a bijective Z-linear map $f^{\prime}: P \rightarrow P^{\prime}$ is a parity preserving lattice isometry if $f^{\prime}$ satisfies the above condition (a), (b), (c) with $f^{\prime}$ in place of $f$.

Lemma 2.2.5. Let $\left(\mathscr{E}, \Pi=\left\{\alpha_{0}, \ldots, \alpha_{n}\right\}, p\right),\left(\mathscr{E}^{\prime}, \Pi^{\prime}=\left\{\alpha_{0}^{\prime}, \ldots, \alpha_{n}^{\prime}\right\}, p^{\prime}\right)$ be affine. Suppose that they are not of a name (AA) $)^{b}$. Denote the root lattice of $\left(\mathscr{E}^{\prime}, \Pi^{\prime}\right.$, $\left.p^{\prime}\right)$ by $P^{\prime}$. Assume that there is a parity preserving lattice isometry $f^{\prime}: P \rightarrow P^{\prime}$. Then there exists a unique parity preserving isometry $f: \mathscr{E} \rightarrow \mathscr{E}^{\prime}$ extending $f^{\prime}$.

Proof. We note that $\left\{\alpha_{0}, \ldots, \alpha_{n}, \Lambda_{0}\right\}$ is a basis of $\mathscr{E}$. On the other hand, it is easily verified that there exists a unique element $\Lambda_{0}^{\prime}$ such that $\left(\Lambda_{0}^{\prime}, f^{\prime}\left(\alpha_{j}\right)\right)=\delta_{0 j}$ and $\left(\Lambda_{0}^{\prime}, \Lambda_{0}^{\prime}\right)=0$. It is easily verified that $\left\{f^{\prime}\left(\alpha_{0}\right), \ldots, f^{\prime}\left(\alpha_{n}\right), \Lambda_{0}^{\prime}\right\}$ form a basis of $\mathscr{E}^{\prime}$. Letting $f\left(\alpha_{j}\right)=f^{\prime}\left(\alpha_{j}\right)$ and $f\left(\Lambda_{0}\right)=\Lambda_{0}^{\prime}, f$ is the desired map. $\quad$ Q. E. D.

The following proposition is known (see [FSS $],[\mathrm{LS}],[\mathrm{LSS}]$ ).

Proposition 2.2.6. Let $\left(\mathscr{E}, \Pi=\left\{\alpha_{0}, \ldots, \alpha_{n}\right\}, p\right)$ and $\left(\mathscr{E}^{\prime}, \Pi^{\prime}=\left\{\alpha_{0}^{\prime}, \ldots, \alpha_{n}^{\prime}\right\}, p^{\prime}\right)$ be data, where $\left(\alpha_{i}, \alpha_{i}\right)=\left(\alpha_{i}^{\prime}, \alpha_{i}^{\prime}\right)=0$ and $p\left(\alpha_{i}\right)=p^{\prime}\left(\alpha_{i}^{\prime}\right)=1$ for a fixed $i$. Suppose that there is a parity preserving isometry $f: \mathscr{E} \rightarrow \mathscr{E}^{\prime}$ such that

$$
f\left(\alpha_{j}\right)=\left\{\begin{aligned}
-\alpha_{i}^{\prime} & \text { if } i=j, \\
\alpha_{j}^{\prime}+\alpha_{i}^{\prime} & \text { if } i \neq j \text { and }\left(\alpha_{i}, \alpha_{j}\right) \neq 0 \\
\alpha_{j}^{\prime} & \text { otherwise. }
\end{aligned}\right.
$$

Put $\mathscr{G}=\mathscr{G}(\mathscr{E}, \Pi, p)$ and $\mathscr{G}^{\prime}=\mathscr{G}\left(\mathscr{E}^{\prime}, \Pi^{\prime}, p^{\prime}\right)$. Then there exists a unique isomorphism $\phi: \mathscr{G} \rightarrow \mathscr{G}^{\prime}$ such that

$$
\begin{aligned}
& \phi\left(H_{\gamma}\right)=H_{f(\gamma)} . \\
& \phi\left(E_{j}\right)=\left\{\begin{aligned}
-(-1)^{p\left(\alpha_{i}\right)} F_{i} & \text { if } i=j, \\
-\frac{(-1)^{p^{\prime}\left(\alpha_{i}^{\prime}\right) p^{\prime}\left(\alpha_{j}^{\prime}\right)}}{\left(\alpha_{i}^{\prime}, \alpha_{j}^{\prime}\right)}\left[E_{j}, E_{i}\right] & \text { if } i \neq j \text { and }\left(\alpha_{i}, \alpha_{j}\right) \neq 0, \\
E_{j} & \text { otherwise. }
\end{aligned}\right.
\end{aligned}
$$




$$
\phi\left(F_{j}\right)=\left\{\begin{aligned}
-E_{i} & \text { if } i=j, \\
-\left[F_{j}, F_{i}\right] & \text { if } i \neq j \text { and }\left(\alpha_{i}, \alpha_{j}\right) \neq 0 \\
F_{j} & \text { otherwise. }
\end{aligned}\right.
$$

In particular,

$$
\phi\left(\mathscr{G}_{\alpha}\right)=\mathscr{G}_{f(\alpha)} \text { for } \alpha \in \Phi(\mathscr{E}, \Pi, p)
$$

Proof. Let $\tilde{\mathscr{G}}=\tilde{\mathscr{G}}(\mathscr{E}, \Pi, p)$. Let $\mathscr{H}^{\prime}$ be the Cartan subalgebra of $\mathscr{G}^{\prime}$. Denote the right hand sides of (2.2.10-11) by $E_{j}^{\prime}$ and $F_{j}^{\prime}$. By using formulas of Lemma 2.2.1, we can show that there is an epimorphism $y: \tilde{\mathscr{G}} \rightarrow \mathscr{G}^{\prime}$ such that $y\left(H_{\gamma}\right)=H_{f(\gamma)}$, $y\left(E_{j}\right)=E_{j}^{\prime}$ and $y\left(F_{j}\right)=F_{j}^{\prime}$. Since $y_{\mid \mathscr{H}}$ is injective, ker $y \cap \mathscr{H}=\{0\}$ and $\tilde{\mathscr{G}} / \operatorname{ker} y$ is an admissible Lie superalgebra such that $\tilde{\mathscr{G}} /$ ker $y>\mathscr{G}$ (see Definition 1.2.1). Let $\bar{y}: \tilde{\mathscr{G}} /$ ker $y \rightarrow \mathscr{G}^{\prime}$ be an isomorphism induced from $y$. By composing of $\bar{y}^{-1}$ and $\Psi[\tilde{\mathscr{G}} /$ ker $y, \mathscr{G}]$, we have an epimorphism $\phi_{1}=\Psi[\tilde{\mathscr{G}} / \operatorname{ker} y, \mathscr{G}] \circ \bar{y}^{-1}: \mathscr{G}^{\prime} \rightarrow \mathscr{G}$ such that $\phi_{1}\left(H_{\gamma}^{\prime}\right)=H_{\gamma}, \phi_{1}\left(E_{j}^{\prime}\right)=E_{j}$ and $\phi_{1}\left(F_{j}^{\prime}\right)=F_{j}$. Since $\phi_{1 \mid \mathscr{H}^{\prime}}=\left(\boldsymbol{y}_{\mid \mathscr{H}}\right)^{-1}$ is injective, $\operatorname{ker} \phi_{1} \cap \mathscr{H}^{\prime}=\{0\}$, which implies $\operatorname{ker} \phi_{1}=\{0\}$. Hence $\phi_{1}$ is an isomorphism, and $\phi$ $=\phi_{1}^{-1}$ is the desired map.

Q.E. D.

We call the parity preserving isometry $f: \mathscr{E} \rightarrow \mathscr{E}^{\prime}$ in Proposition 2.2.6 the superreflection (see [LSS]) corresponding to the simple root $\alpha_{i}$ with $\left(\alpha_{i}, \alpha_{i}\right)=0$.

Proposition 2.2.7. Let $\left(\mathscr{E}, \Pi=\left\{\alpha_{0}, \ldots, \alpha_{n}\right\}, p\right)$ be a datum. Let $\alpha_{i} \in \Pi$ be such that $\left(\alpha_{i}, \alpha_{i}\right) \neq 0$ and $a_{i j}=\frac{2\left(\alpha_{i}, \alpha_{j}\right)}{\left(\alpha_{t}, \alpha_{t}\right)} \in-\mathbb{Z}_{+}$for $j \neq i$. Assume that $-a_{i j} \in 2 \mathbb{Z}_{+}$if $p\left(\alpha_{i}\right)=$ 1. Let $f: \mathscr{E} \rightarrow \mathscr{E}$ be a parity preserving isometry defined by $f(\nu)=\nu-\frac{2\left(\nu, \alpha_{i}\right)}{\left(\alpha_{i}, \alpha_{i}\right)} \alpha_{i}$. Suppose that $\mathscr{G}^{\#}=\mathscr{G}^{\#}(\mathscr{E}, \Pi, p)$ is an admissible Lie superalgebra such that the following equalities hold.

$$
\operatorname{ad}\left(E_{i}\right)^{1-a_{i j}}\left(E_{j}\right)=\operatorname{ad}\left(F_{i}\right)^{1-a_{i j}}\left(F_{j}\right)=0 \text { for any } j \neq i
$$

Put

$$
\phi=\left\{\begin{aligned}
\exp \left(\operatorname{ad} E_{i}\right) \exp \left(-\frac{2}{\left(\alpha_{i}, \alpha_{i}\right)} \operatorname{ad} F_{i}\right) \exp \left(\operatorname{ad} E_{i}\right) & \text { if } p\left(\alpha_{i}\right)=0, \\
\exp \left(\operatorname{ad}\left[E_{i}, E_{i}\right]\right) \exp \left(\frac{-1}{2\left(\alpha_{i}, \alpha_{i}\right)} \operatorname{ad}\left[F_{i}, F_{i}\right]\right) \exp \left(\operatorname{ad}\left[E_{i}, E_{i}\right]\right) & \text { if } p\left(\alpha_{i}\right)=1 .
\end{aligned}\right.
$$

Then $\phi$ is well-defined as an automorphism of $\mathscr{G}^{\#}$, and satisfies:

$$
\phi\left(H_{\nu}\right)=H_{f(\nu)}
$$

In particular, 


$$
\phi\left(\mathscr{G}_{\beta}^{\#}\right)=\mathscr{G}_{f}^{\sharp}(\beta) \quad \text { for } \quad \beta \in \Phi\left[\mathscr{G}^{\#}(\mathscr{E}, \Pi, p)\right] .
$$

Proof. The proposition follows from the same argument used in $[\mathrm{K} 1, \S 3.8]$. Q. E. D.

We call the parity preserving isometry $f: \mathscr{E} \rightarrow \mathscr{E}$ in Proposition 2.2.7 the reflection corresponding to a simple root $\alpha_{i}$ with $\left(\alpha_{i}, \alpha_{i}\right) \neq 0$.

Along the same line as Proposition 2.2.6, we have

Proposition 2.2.8. Let $\left(\mathscr{E}, \Pi=\left\{\alpha_{0}, \ldots, \alpha_{n}\right\}, p\right)$ be datum. Assume that there is a parity preserving isometry $f: \mathscr{E} \rightarrow \mathscr{E}$ satisfying $f(\Pi)=\Pi$. Put $\mathscr{G}=\mathscr{G}(\mathscr{E}, \Pi, p)$. Then there exists a unique isomorphism $\phi: \mathscr{G} \rightarrow \mathscr{G}$ such that $\phi\left(H_{\gamma}\right)=H_{f(\gamma)}$. In particular, $\phi\left(\mathscr{G}_{\alpha}\right)=\mathscr{G}_{f(\alpha)}(\beta \in \Phi(\mathscr{E}, \Pi, p))$.

We call the parity preserving isometry $f: \mathscr{E} \rightarrow \mathscr{E}$ in Proposition 2.2.8 the diagram automorphism.

2.3. Keep the notation in 1.4-5. Let $\left(\mathscr{E}, \Pi=\left\{\alpha_{0}, \ldots, \alpha_{n}\right\}, p\right)$ be a datum of affine ABCD type. As discussed in 1.4-5, the C-linear space $\mathscr{E}$ is a subspace of $\mathscr{E}$ ex of codimension one if $(\mathscr{E}, \Pi, p)$ is $(\mathrm{AA})^{g}$. Otherwise $\mathscr{E}=\mathscr{E}^{e x}$. The C-linear space $\mathscr{E}^{e x}$ has a basis $\left\{\bar{\varepsilon}_{1}, \ldots, \bar{\varepsilon}_{N}, \delta, \Lambda_{0}\right\}$ which consists of the fundamental elements. We note that $\left(\bar{\varepsilon}_{i}, \bar{\varepsilon}_{j}\right)=\delta_{i j} \bar{d}_{i}\left(\bar{d}_{i}= \pm 1\right)$. We also note that $n=N-1$ if $(\mathscr{E}, \Pi, p)$ is (AA), and $n=N$ otherwise. Moreover the name of the Dynkin diagram of $(\mathscr{E}, \Pi, p)$ has been given; it is (AA), (BB), (CB), (DB), (CC), (CD), (DC) or (DD). For a fixed positive integer $N, \operatorname{ABCD}(N)$ has been defined as the set of data $\{(\mathscr{E}, \Pi, p)\}$ of affine ABCD-type such that $n=N-1$ if $(\mathscr{E}, \Pi, p)$ is (AA), and $n=N$ otherwise.

Definition 2.3.1. Let $(\mathscr{E}, \Pi, p) \in \operatorname{ABCD}(N)$. For a fixed $i, 0 \leq i \leq n$, we define $\left({ }^{s_{i}} \mathscr{E},{ }^{s_{i}} \Pi,{ }^{s_{i}} p\right) \in \mathrm{ABCD}(N)$ by (a)-(d) below:

(a ) Let $\left\{\bar{\varepsilon}_{1}^{\prime}, \ldots, \bar{\varepsilon}_{N}^{\prime}, \delta, \Lambda_{0}\right\}$ be the fundamental elements of $\left({ }^{s_{i}} \mathscr{E},{ }^{s_{i}} \Pi,{ }^{s_{i}} p\right)$, and $\bar{d}_{i}^{\prime}=\left(\bar{\varepsilon}_{i}^{\prime}, \bar{\varepsilon}_{i}^{\prime}\right)= \pm 1$.

(b) If $1 \leq i \leq N-1$ and $\left(\alpha_{i}, \alpha_{i}\right)=0$, then let $\left({ }^{s_{i}} \mathscr{E},{ }^{s_{i}} \Pi,{ }^{s_{i}} p\right)$ be such that $\bar{d}_{i}^{\prime}=$ $\bar{d}_{i+1}, \bar{d}_{i+1}^{\prime}=\bar{d}_{i}, \bar{d}_{j}^{\prime}=\bar{d}_{j}(j \neq i, i+1)$ and the name $(\mathrm{XY})$ of the Dynkin diagram of $\left({ }^{s_{i}} \mathscr{E},{ }^{s_{i}} \Pi,{ }^{s_{i}} p\right)$ is

$$
\begin{cases}(\mathrm{DY}) & \text { if } i=1 \text { and } \mathrm{X}=\mathrm{C} \\ (\mathrm{CY}) & \text { if } i=1 \text { and } \mathrm{X}=\mathrm{D} \\ (\mathrm{XD}) & \text { if } i=N-1 \text { and } \mathrm{Y}=\mathrm{C} \\ (\mathrm{XC}) & \text { if } i=N-1 \text { and } \mathrm{Y}=\mathrm{D} \\ (\mathrm{XY}) & \text { otherwise. }\end{cases}
$$


(Here X, Y denotes A, B, C or D.)

(c) If $(\mathscr{E}, \Pi, p)$ has the name (AA), and if $i=0$ and $\left(\alpha_{0}, \alpha_{0}\right)=0$, then let $\left({ }^{s_{i}} \mathscr{E},{ }^{s_{i}} \Pi,{ }^{s_{i}} p\right)$ be such that $\bar{d}_{1}^{\prime}=\bar{d}_{N}, \bar{d}_{N}^{\prime}=\bar{d}_{1}, \bar{d}_{j}^{\prime}=\bar{d}_{j}(j \neq 1, N)$ and $\left({ }^{s} \mathscr{E},{ }^{s_{i}} \Pi,{ }^{s_{i}} p\right)$ also has the name (AA).

(d) If $(\mathscr{E}, \Pi, p)$ and $0 \leq i \leq n$ does not satisfy the hypotheses of (b) nor (c), then let $\left({ }^{s_{i}} \mathscr{E},{ }^{s} \Pi,{ }^{s_{i}} p\right)=(\mathscr{E}, \Pi, p)$.

2.4.

Definition 2.4.1. For $(\mathscr{E}, \Pi, p) \in \operatorname{ABCD}(N)$, define $\left(\mathscr{E}^{\dagger}, \Pi^{\dagger}\right)$ by (a) and (b) below.

(a ) Let $\mathscr{E}^{e x}$ be as in 1.4. Let $\mathscr{E}^{\mathscr{E}^{e x}}$ be an $(N+2)$-dimensional C-linear space with a basis $\left\{\varepsilon_{1}, \ldots, \varepsilon_{N}, \delta^{\dagger}, \Lambda_{0}^{\dagger}\right\}$ and a symmetric form $($,$) such that \left\{\varepsilon_{1}, \ldots, \varepsilon_{N}\right.$, $\left.\frac{1}{\sqrt{2}}\left(\delta^{\dagger}+\Lambda_{0}^{\dagger}\right), \frac{\sqrt{-1}}{\sqrt{2}}\left(\delta^{\dagger}-\Lambda_{0}^{\dagger}\right)\right\}$ is a orthonormal basis of $\mathscr{E}^{+e x}$ with respect to $($,$) .$ Define the C-linear isomorphism $\iota: \mathscr{E}^{e x} \rightarrow \mathscr{E}^{\mathscr{T}^{+e x}}$ by $\iota\left(\bar{\varepsilon}_{i}\right)=\varepsilon_{i}, \iota(\delta)=\delta^{\dagger}, \iota\left(\Lambda_{0}\right)=\Lambda_{0}^{\dagger}$. Define a subspace $\mathscr{E}^{\dagger}$ of $\mathscr{E}^{\dagger}$ by $\mathscr{E}^{\dagger}=\iota(\mathscr{E})$.

(b) Define $\Pi^{\dagger}=\left\{\alpha_{0}^{\dagger}, \alpha_{1}^{\dagger}, \ldots, \alpha_{n}^{\dagger}\right\}$ by

$$
\alpha_{i}^{\dagger}= \begin{cases}\delta^{\dagger}-2 \varepsilon_{1} & \text { if } i=0 \text { and }(\mathscr{E}, \Pi, p) \text { is (DB), (DC), or (DD), } \\ 2 \varepsilon_{N} & \text { if } i=N \text { and }(\mathscr{E}, \Pi, p) \text { is (CD), or (DD), } \\ \iota\left(\alpha_{i}\right) & \text { otherwise. }\end{cases}
$$

We call $\left(\mathscr{E}^{\dagger}, \Pi^{\dagger}\right)$ the auxiliary datum of $(\mathscr{E}, \Pi, p)$. We also give a name (XY) to a Dynkin diagram of $\left(\mathscr{E}^{\dagger}, \Pi^{\dagger}\right)$ as follows:

$$
\begin{cases}(\mathrm{AA}) & \text { if }(\mathscr{E}, \Pi, p) \text { is (AA), } \\ (\mathrm{BB}) & \text { if }(\mathscr{E}, \Pi, p) \text { is (BB), } \\ (\mathrm{CB}) & \text { if }(\mathscr{E}, \Pi, p) \text { is (CB) or (DB), } \\ (\mathrm{CC}) & \text { if }(\mathscr{E}, \Pi, p) \text { is (CC), (CD), (DC) or (DD). }\end{cases}
$$

Put $P_{+}^{\dagger}=\mathbf{Z}_{+} \alpha_{0}^{\dagger} \oplus \cdots \oplus \mathbf{Z}_{+} \alpha_{n}^{\dagger}, P_{-}^{\dagger}=-P_{+}^{\dagger}$ and $P^{\dagger}=P_{+}^{\dagger}+P_{-}^{\dagger}$.

Lemma 2.4.2. (i) $\iota\left(P_{+}\right) \subset P_{+}^{\dagger}, \iota(P)=P^{\dagger}$.

(ii) $(x, x) \geq 0$ for any $x \in P^{\dagger}$. Moreover $x \in P^{\dagger}$ satisfies $(x, x)=0$ if and only if $x \in \mathbf{Z} \delta^{\dagger}$.

(iii) $\left(\left(\left(^{s_{i}} \mathscr{E}\right)^{\dagger},\left({ }^{s_{i}} \Pi\right)^{\dagger}\right)=\left(\mathscr{E}^{\dagger}, \Pi^{\dagger}\right)\right.$.

Proof. Clear.

Q. E. D.

Proposition 2.4.3. Let $(\mathscr{E}, \Pi, p) \in \mathrm{ABCD}(N)$ and $0 \leq i \leq n$. Then there exists 
a parity preserving isometry $s_{i}: \mathscr{E}^{s_{i}}{ }^{\mathscr{E}}$ such that

$$
\iota\left(s_{i}(v)\right)=\iota(v)-\frac{2\left(\iota(v), \alpha_{i}^{\dagger}\right)}{\left(\alpha_{i}^{\dagger}, \alpha_{i}^{\dagger}\right)} \alpha_{i}^{\dagger}
$$

for $v \in P$. Moreover, if $(\mathscr{E}, \Pi, p)$ is not $(\mathrm{AA})^{b}$, such $s_{i}$ is unique.

Proof. The existence of $s_{i}$ can be verified easily. The latter half of the statement is clear from Lemma 2.2.5.

Q. E. D.

2.5. Since $s_{i}$ defined in Proposition 2.4.3 is a super-reflection, a reflection or a diagram automorphism, by Propositions 2.2.6-8, we have:

Theorem 2.5.1. Let $(\mathscr{E}, \Pi, p) \in \operatorname{ABCD}(N)$, and let $i$ be $0 \leq i \leq n$.

(i) There is an isomorphism $L_{i}: \mathscr{G}(\mathscr{E}, \Pi, p) \rightarrow \mathscr{G}\left({ }^{s_{i}} \mathscr{E},{ }^{s} \Pi,{ }^{s_{i}} p\right)$ such that:

$$
L_{i}\left(H_{\gamma}\right)=H_{s_{i}(\gamma)} \quad(\gamma \in \mathscr{E}) .
$$

In particular, $L_{i}$ satisfies:

$$
L_{i}\left(\mathscr{G}_{\alpha}\right)=\mathscr{G}\left({ }^{s_{i}} \mathscr{E},{ }^{s_{i}} \Pi,{ }^{s_{i}} p\right)_{s_{i}(\alpha)} \quad(\alpha \in \Phi)
$$

(ii) Let $L_{i}$ and $L_{i}^{\prime}$ be two isomorphisms satisfying (2.5.1). Then $L_{i} \equiv L_{i}^{\prime}$ (see Definition 1.2.5).

Remark 2.5.2. Let $s_{i}$ be as in Proposition 2.4.3. If $s_{i}$ is a super-reflection, then it always changes Dynkin diagram. For example:
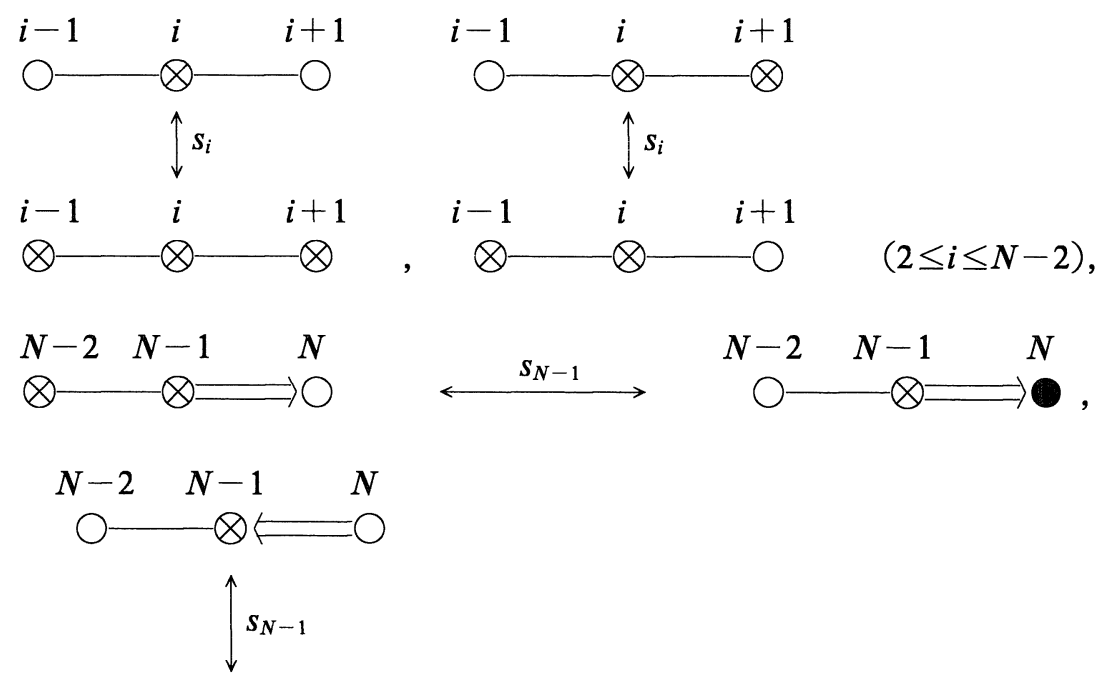

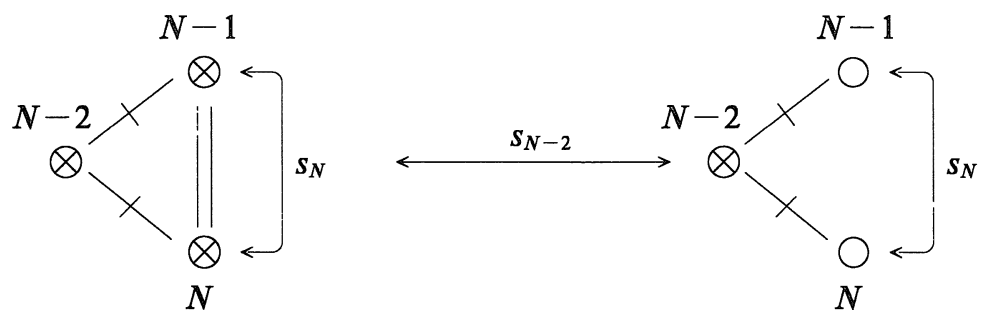

(The two $s_{N}$ of the undermost Dynkin diagrams are not super-reflections but diagram automorphisms.)

2.6. Let $(\mathscr{E}, \Pi, p) \in \operatorname{ABCD}(N)$. Let $h t^{\dagger}: P_{+}^{\dagger} \rightarrow \mathbb{Z}_{+}$be a function defined by $h t^{\dagger}\left(\sum_{i=0}^{n} k_{i} \alpha_{i}^{\dagger}\right)=\sum_{i=0}^{n} k_{i}$.

For a composition $w=s_{i(1)} s_{i(2)} \cdots s_{i(r-1)} s_{i(r)}$ of $s_{i(k)}$ 's, define $\left({ }^{w} \mathscr{E},{ }^{w} \Pi,{ }^{w} p\right)$ by

$$
\begin{aligned}
&{ }^{w} \mathscr{E}={ }^{s_{i}(1)}\left(\ldots{ }^{s_{i(r-1)}}\left({ }^{s_{i(r)}} \mathscr{E}\right) \cdots\right), \\
&{ }^{w} \Pi={ }^{s_{i(1)}}\left(\ldots{ }^{s_{i(r-1)}}\left({ }^{s_{i(r)}} \Pi\right) \cdots\right), \\
&{ }^{w} p={ }^{s_{i(1)}}\left(\ldots{ }^{s_{i(r-1)}}\left({ }^{s_{i(r)}} p\right) \cdots\right) .
\end{aligned}
$$

We note that $w$ is a parity preserving isometry from $\mathscr{E}$ to ${ }^{w} \mathscr{E}$. Let ${ }^{w} P,{ }^{w} P_{+},{ }^{w} P_{-}$ denote the root lattice, the positive and negative parts of the root lattice of $\left({ }^{w} \mathscr{E},{ }^{w} \Pi\right.$, ${ }^{w} p$ ) respectively. We note that the identity map $e=i d_{\mathscr{E}}: \mathscr{E} \rightarrow \mathscr{E}$ is also a composition of $s_{i}$ 's.

Proposition 2.6.1. Let $(\mathscr{E}, \Pi, p) \in \operatorname{ABCD}(N)$. Keep the above notation.

(i) Let $\alpha \in \Phi(\mathscr{E}, \Pi, p) \backslash \mathbb{Z} \delta$. Then there exists a w such that $w(\alpha) \in \mathbb{Z} \beta \cap$ $\Phi\left({ }^{w} \mathscr{E},{ }^{w} \Pi,{ }^{w} p\right)$ for some $\beta \in{ }^{w} \Pi$.

(ii) Let $\alpha \in\left(P_{+} \cup P_{-}\right) \backslash(\Phi(\mathscr{E}, \Pi, p) \cup \mathbb{Z} \delta)$. Then one of the following cases occurs:

Case 1. There exists a $w$ such that $w(\alpha) \in{ }^{w} P \backslash\left({ }^{w} P_{+} \cup{ }^{w} P_{-}\right)$.

Case 2. There exists a wuch that $w(\alpha) \in \mathbb{Z} \beta \backslash \Phi\left({ }^{w} \mathscr{E},{ }^{w} \Pi,{ }^{w} p\right)$ for some $\beta \in{ }^{w} \Pi$.

In particular $w(\alpha) \notin \Phi\left({ }^{w} \mathscr{E},{ }^{w} \Pi,{ }^{w} p\right)$.

Proof. We prove (i) and (ii) simultaneously. We may assume $\alpha \in \mathcal{P}_{+} \backslash \mathbb{Z} \delta$. We may also assume that $\alpha \notin \mathbb{Z} \alpha_{j}$ for any $\alpha_{j} \in \Pi$. These assumptions imply that $\alpha$ $\notin \in \mathbb{Z}_{\iota}^{-1}\left(\alpha_{j}^{\dagger}\right)$ for any $\alpha_{j} \in \Pi$. Since $\alpha \not \subset \mathbb{Z} \delta$, by Lemma 2.4 .2 (ii), we have ( $\iota(\alpha)$, $\iota(\alpha))>0$. By Lemma 2.4.2 (i), there exists an $\alpha_{i}^{\dagger} \in \Pi^{\dagger}$ such that $\left(\iota(\alpha), \alpha_{i}^{\dagger}\right)>0$. Since $\left(\iota\left(s_{i}(\alpha)\right), \iota\left(s_{i}(\alpha)\right)\right)=(\iota(\alpha), \iota(\alpha))>0, s_{i}(\alpha) \in^{s_{i}} P \backslash \mathbb{Z} \delta$ (see Lemma 2.4.2 (ii)). Since $\alpha \notin \subset \mathbf{Z}^{-1}\left(\alpha_{i}\right)$, we see that $s_{i}(\alpha)=\alpha-\frac{2\left(\alpha_{t}^{\dagger}, \iota(\alpha)\right)}{\left(\alpha_{i}^{\dagger}, \alpha_{i}^{\dagger}\right)} \iota^{-1}\left(\alpha_{i}^{\dagger}\right) \notin^{s_{i}} P_{-} \cup \mathbb{Z} \delta$, and that, if $s_{i}(\alpha) \in^{s_{i}} P_{+} \backslash \mathbb{Z} \delta, h t^{\dagger}\left(\iota\left(s_{i}(\alpha)\right)<h t^{\dagger}(\iota(\alpha))\right.$. Thus we can get a finite sequence 
$\left\{w_{0}=\mathrm{id}, w_{j}=s_{i(j)} \cdots s_{i(2)} s_{i(1)}(j=1, \ldots, k)\right\}$ such that

$$
w_{j}(\alpha) \in\left({ }^{w_{j}} \boldsymbol{P}_{+} \backslash \mathbf{Z} \delta\right), \quad h t^{\dagger}\left(\iota\left(w_{j}(\alpha)\right)\right)<h t^{\dagger}\left(\iota\left(w_{j-1}(\alpha)\right)\right),
$$

and that, letting $w=w_{k}$, we have either

(a) $w(\alpha) \in \mathbf{Z} \beta \quad$ for some $\beta \in{ }^{w} \Pi$,

or

(b ) $\quad w(\alpha) \not \mathbb{N}^{w} P_{+} \cup^{w} P_{-}$.

Put $^{w_{j}} \mathscr{G}=\mathscr{G}\left({ }^{w_{j}} \mathscr{E},{ }^{w_{j}} \Pi,{ }^{w_{j}} p\right)$. By Theorem 2.5.1, $\operatorname{dim}{ }^{w_{j}} \mathscr{G}_{w_{j}(\alpha)}=\operatorname{dim} L_{j}\left({ }^{w_{j-1}} \mathscr{G}_{w_{j-1}(\alpha)}\right)$ $=\operatorname{dim}^{w_{j-1}} \mathscr{G}_{w_{j-1}(\alpha)}$. By Lemma 1.2.3, $\operatorname{dim} \mathscr{G}_{\alpha}=\operatorname{dim}{ }^{w} \mathscr{G}_{w(\alpha)} \neq 0$ (i.e. $\left.=1\right)$ if and only if the condition (a) and the condition $w(\alpha) \in \Phi\left({ }^{w} \mathscr{E},{ }^{w} \Pi,{ }^{w} p\right)$ hold. $\quad$ Q. E. D.

Remark 2.6.2. For $(\mathrm{XY})=(\mathrm{AA}),(\mathrm{BB}),(\mathrm{CB})$ or $(\mathrm{CC})$, let $\operatorname{ABCD}(N)_{(\mathrm{XY})}$ be the set of $(\mathscr{E}, \Pi, p) \in \mathrm{ABCD}(N)$ such that $\left(\mathscr{E}^{\dagger}, \Pi^{\dagger}\right)$ is $(\mathrm{XY})$. Let $\mathscr{E}(\mathrm{XY})=$ $\oplus \mathscr{E}\left((\mathscr{E}, \Pi, p) \in \mathrm{ABCD}(N)_{(\mathrm{XY})}\right)$. Then a set of suitably chosen $s_{i}$ 's gives an action of the affine Weyl group of $\left(\mathscr{E}^{\dagger}, \Pi^{\dagger}\right)$ on $\mathscr{E}(\mathrm{XY})$, i.e. $s_{i}^{2}=\mathrm{id}: \mathscr{E} \rightarrow \mathscr{E}$, and $\left(s_{i} s_{j}\right)^{h(i, j)}=$ id: $\mathscr{E} \rightarrow \mathscr{E}$ where $h(i, j)=2,3,4,6$ if $\frac{4\left(\alpha_{1}^{\dagger}, \alpha_{j}^{\dagger}\right)^{2}}{\left(\alpha_{1}^{\dagger}, \alpha_{t}^{\dagger}\right)\left(\alpha_{j}^{\dagger}, \alpha_{j}^{\dagger}\right)}=0,1,2,3$ respectively.

\section{§3. A Maximal Affine-Admissible Algebra}

3.1. By Lemma 1.2.3, Theorem 2.5.1 and Proposition 2.6.1, we have

Proposition 3.1.1. Let $(\mathscr{E}, \Pi, p) \in \operatorname{ABCD}(N)$, and $\mathscr{G}=\mathscr{G}(\mathscr{E}, \Pi, p)$. Then, for $\alpha \in \Phi(\mathscr{E}, \Pi, p) \backslash \mathbf{Z} \delta, \operatorname{dim} \mathscr{G}_{\alpha}=1$.

Definition 3.1.2. (i) Let $(\mathscr{E}, \Pi, p) \in \operatorname{ABCD}(N)$. Let $\mathscr{G}^{\natural \#}=\mathscr{G}^{\natural \#}(\mathscr{E}, \Pi, p)$ be an admissible Lie superalgebra with respect to $(\mathscr{E}, \Pi, p)$. Namely $\mathscr{G}^{\natural \sharp}(\mathscr{E}, \Pi, p)>$ $\mathscr{G}=\mathscr{G}(\mathscr{E}, \Pi, p)$. We say that $\mathscr{G}^{\natural \#}=\mathscr{G}^{\sharp}(\mathscr{E}, \Pi, p)$ is affine-admissible if the following conditions hold.

$$
\begin{aligned}
& \text { (ia) } \Phi\left[\mathscr{G}^{\sharp \sharp}(\mathscr{E}, \Pi, p)\right]=\Phi(\mathscr{E}, \Pi, p), \\
& \text { (ib) } \operatorname{dim} \mathscr{G}_{\alpha}^{\natural \sharp}=1 \quad \text { for any } \alpha \in \Phi(\mathscr{E}, \Pi, p) \backslash \mathbf{Z} \delta .
\end{aligned}
$$

(ii) Let $(\mathscr{E}, \Pi, p) \in \operatorname{ABCD}(N)$, and let $A I=A I(\mathscr{E}, \Pi, p)$ be the set of affine -admissible Lie superalgebras with respect to $(\mathscr{E}, \Pi, p)$. Let $\mathscr{G}^{\natural}=\mathscr{G}^{\natural}(\mathscr{E}, \Pi, p)$ be an admissible Lie superalgebra defined by

$$
\mathscr{G}^{\natural}=\vee_{\mathscr{G}^{\natural \sharp} \in A I} \mathscr{G}^{\natural \sharp} \text {. }
$$


(see 1.2). Then $\mathscr{G}^{\natural}$ is a unique maximal affine-admissible Lie superalgebra in $A I(\mathscr{E}$, $\Pi, p)$. Namely $\mathscr{G}^{\natural} \in A I$, and $\mathscr{G}^{\natural}>\mathscr{G}^{\natural \#}$ for any $\mathscr{G}^{\natural \sharp} \in A I$. By (1.2.4) and (3.1.1-2), and by Proposition 3.1.1, we have

$$
\operatorname{dim} \mathscr{G}_{\alpha}^{\natural}=\operatorname{dim} \mathscr{G}_{\alpha} \quad \text { if } \quad \alpha \in\left(\left(\boldsymbol{P}_{+} \cup \boldsymbol{P}_{-}\right) \backslash \mathbf{Z} \delta\right) \cup\{0\}
$$

Proposition 3.1.3. Let $(\mathscr{E}, \Pi, p) \in \operatorname{ABCD}(N)$. If $(\delta, \rho) \neq 0, \mathscr{G}^{\natural}(\mathscr{E}, \Pi, p)$ coincides with $\mathscr{G}(\mathscr{E}, \Pi, p)$.

Proof. Since $(\delta, \delta)=0,(\delta, \rho) \neq 0$ implies that $2(k \delta, \rho) \neq(k \delta, k \delta)$ for $k \neq 0$. Since $\delta \in P_{+}$, by Proposition 1.2.2 and (3.1.3), we have $\operatorname{dim} \mathscr{G}_{k \delta}^{\natural}=\operatorname{dim} \mathscr{G}_{k \delta}$ for $k>$ 0 . By symmetry, we also have $\operatorname{dim} \mathscr{G}_{k \delta}^{\natural}=\operatorname{dim} \mathscr{G}_{k \delta}$ for $k<0$. Hence $\mathscr{G}^{\natural}(\mathscr{E}, \Pi, p)$ coincides with $\mathscr{G}(\mathscr{E}, \Pi, p)$.

Q.E.D.

Example 3.1.4. Here we give two examples when $(\delta, \rho)=0$, and explain what happens in each case. The first example is $\mathscr{G}_{1}$ such that its Dynkin diagram is (AA) with $N=4$ and its parity is given by $p\left(\alpha_{1}\right)=p\left(\alpha_{3}\right)=0$ and $p\left(\alpha_{0}\right)=p\left(\alpha_{2}\right)=1$. Then $\mathscr{G}_{1}=\left(\mathrm{A}(1,1)^{(1)}\right)^{\mathscr{H}}$ and $\mathscr{G}_{1}^{\natural}=\left(s l(2,2)^{(1)}\right)^{\mathscr{H}}$. In particular, $\mathscr{G}_{1} \neq \mathscr{G}_{1}^{\natural}$. We also note that $\operatorname{dim}\left(\mathscr{G}_{1}\right)_{k \delta}=2$ and $\operatorname{dim}\left(\mathscr{G}_{1}^{\natural}\right)_{k \delta}=3$ hold for $k \neq 0$. The second example is $\mathscr{G}_{2}$ such that its Dynkin diagram is (CD) with $N=3$ and its parity is given by $p\left(\alpha_{0}\right)=$ $p\left(\alpha_{2}\right)=p\left(\alpha_{3}\right)=0$ and $p\left(\alpha_{1}\right)=1$. Then $\mathscr{G}_{2}^{\natural}$ coincides with $\mathscr{G}_{2}$, and $\mathscr{G}_{2}=\mathbf{D}(2,1)^{(1)}$. In Theorem 3.5.1, we shall show when $\mathscr{G}^{\natural}(\mathscr{E}, \Pi, p)$ does not coincide with $\mathscr{G}(\mathscr{E}, \Pi$, p) for $(\mathscr{E}, \Pi, p) \in \mathrm{ABCD}(N)$.

Proposition 3.1.5. Let $(\mathscr{E}, \Pi, p) \in \operatorname{ABCD}(N)$. Put $\mathscr{G}=\mathscr{G}(\mathscr{E}, \Pi, p), \mathscr{G}^{\natural}=$

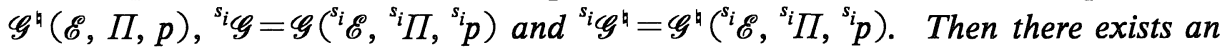
isomorphism $L_{i}^{\natural}: \mathscr{G}^{\natural} \rightarrow^{s_{i}} \mathscr{G}^{\natural}$ such that the following diagram commutes.

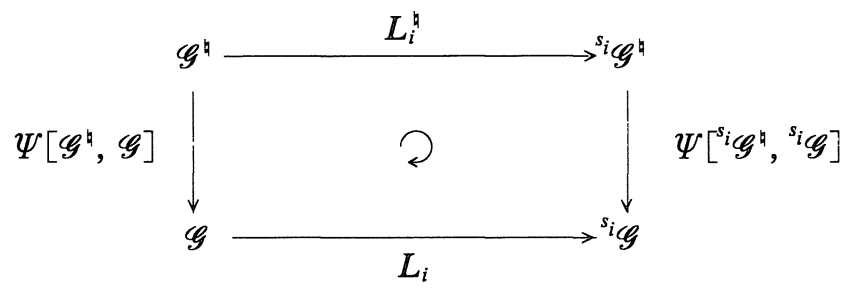

Proof. If $\iota\left(\alpha_{i}\right)=\alpha_{i}^{\dagger}$ and $\left(\alpha_{i}, \alpha_{i}\right) \neq 0$, use Proposition 2.2.7.

Assume $\iota\left(\alpha_{i}\right)=\alpha_{i}^{\dagger}$ and $\left(\alpha_{i}, \alpha_{i}\right)=0$. By $2 \alpha_{i} \not \subset \Phi(\mathscr{E}, \Pi, p),\left[E_{i}, E_{i}\right]=\left[F_{i}, F_{i}\right]=$ 0 . Hence, by formulas in Lemma 2.2.1, there is an epimorphism $y^{\natural}: \tilde{\mathscr{G}}(\mathscr{E}, \Pi, p) \rightarrow$ ${ }^{s_{i}} \mathscr{G}^{\natural}$ such that $\left.\Psi{ }^{s_{i}} \mathscr{G}^{\natural},{ }^{s_{i}} \mathscr{G}\right] \circ y^{\natural}$ coincides with $y$ in the proof of Proposition 3.2.7. It is clear that $\tilde{\mathscr{G}}(\mathscr{E}, \Pi, p) / \operatorname{ker} y^{\natural}$ is affine-admissible with respect to $(\mathscr{E}, \Pi, p)$. By the maximality of ${ }^{s_{i}} \mathscr{G}^{\natural}$, we see that $\tilde{\mathscr{G}}(\mathscr{E}, \Pi, p) /$ ker $y^{\natural}=\mathscr{G}^{\natural}$.

Similarly we can prove the proposition in the case that $\iota\left(\alpha_{i}\right) \neq \alpha_{i}^{\dagger}$. Q. E. D. 
3.2. For $N \geq 3$, let $\mathrm{CD}(N)$ be the set of data $(\mathscr{E}, \Pi, p) \in \operatorname{ABCD}(N)$ whose Dynkin diagrams are (CC), (CD), (DC) or (DD). For $(\mathscr{E}, \Pi, p) \in \mathrm{CD}(N)$ and a fixed $1 \leq i \leq N$, let $\mathscr{E}_{[i]}=\left\{x \in \mathscr{E} \mid\left(x, \bar{\varepsilon}_{i}\right)=0\right\}$. Put $\theta_{j}=\bar{\varepsilon}_{j}$ if $1 \leq j \leq i-1$, and $\theta_{j-1}=$ $\bar{\varepsilon}_{\mathrm{j}}$ if $i+1 \leq j \leq N$. Then $\left\{\theta_{1}, \ldots, \theta_{N-1}, \delta, \Lambda_{0}\right\}$ is a basis of $\mathscr{E}_{[i]}$. Let ${ }_{0} \Pi_{[i]}=\left\{\theta_{j}-\theta_{j+1}\right.$ $(1 \leq j \leq N-2)\},{ }_{1} \Pi_{[i]}={ }_{0} \Pi_{[i]} \cup\left\{\delta-2 \theta_{1}, 2 \theta_{N-1}\right\},{ }_{2} \Pi_{[i]}={ }_{0} \Pi_{[i]} \cup\left\{\delta-2 \theta_{1}, \theta_{N-2}-\right.$ $\left.\theta_{N-1}\right\},{ }_{3} \Pi_{[i]}={ }_{0} \Pi_{[i]} \cup\left\{\delta-\theta_{1}-\theta_{2}, 2 \theta_{N-1}\right\},{ }_{4} \Pi_{[i]}={ }_{0} \Pi_{[i]} \cup\left\{\delta-\theta_{1}-\theta_{2}, \theta_{N-2}-\theta_{N-1}\right\}$.

Put

$$
\Pi_{[i]}= \begin{cases}{ }_{1} \Pi_{[i]} & \text { if }{ }_{1} \Pi_{[i]} \subset \Phi(\mathscr{E}, \Pi, p), \\ { }_{2} \Pi_{[i]} & \text { if }{ }_{1} \Pi_{[i]} \subset \Phi(\mathscr{E}, \Pi, p) \text { and }{ }_{2} \Pi_{[i]} \subset \Phi(\mathscr{E}, \Pi, p), \\ { }_{3} \Pi_{[i]} & \text { if }{ }_{1} \Pi_{[i]} \subset \Phi(\mathscr{E}, \Pi, p) \text { and }{ }_{3} \Pi_{[i]} \subset \Phi(\mathscr{E}, \Pi, p), \\ { }_{4} \Pi_{[i]} & \text { if }{ }_{1} \Pi_{[i]},{ }_{2} \Pi_{[i]},{ }_{3} \Pi_{[i]} \subset \Phi(\mathscr{E}, \Pi, p) \text { and }{ }_{4} \Pi_{[i]} \subset \Phi(\mathscr{E}, \Pi, p) .\end{cases}
$$

Then $\Pi_{[i]}$ is always well defined, and $\Pi_{[i]}$ consists of $N$ linearly independent elements. Then a triple $\left(\mathscr{E}_{[i]}, \Pi_{[i]}, p_{[i]}\right)$ is a datum where $p_{[i]}=p_{\mid I_{[i]}}$.

Definition 3.2.1. Let $N \geq 3$ and $(\mathscr{E}, \Pi, p) \in \mathrm{CD}(N)$.

(i) We say that $(\mathscr{E}, \Pi, p)$ is $i$-good if one of the following conditions holds.

(ia) Any element $\beta \in \Pi_{[i]}$ satisfies $p(\beta)=0$, and the Dynkin diagram corresponding to $(\mathscr{E}, \Pi, p)$ is well defined (see 1.3) and connected.

(ib) The datum $(\mathscr{E}, \Pi, p)$ satisfies $N \geq 4$, and there is a parity preserving isometry $f: \mathscr{E}_{[i]} \rightarrow \mathscr{E}^{\prime}$ for some $\left(\mathscr{E}^{\prime}, \Pi^{\prime}, p^{\prime}\right) \in \mathrm{CD}(N-1)$ such that $f\left(\theta_{j}\right)=\bar{\varepsilon}_{j}, f(\delta)=$ $\delta$ and $f\left(\Lambda_{0}\right)=\Lambda_{0}$.

(ic) The datum $(\mathscr{E}, \Pi, p)$ satisfies $N=3$, and there is an isomorphism $f$ : $\mathscr{G}\left(\mathscr{E}_{[i]}, \Pi_{[i]}, p_{[i]}\right) \rightarrow \mathscr{G}\left(\mathscr{E}^{\prime}, \Pi^{\prime}, p^{\prime}\right)$ for some $\left(\mathscr{E}^{\prime}, \Pi^{\prime}, p^{\prime}\right)$ of $(\mathrm{AA})^{g}$ with $N=3$ such that $f\left(E_{j}\right)=\frac{1}{2} E_{j}$ and $f\left(F_{j}\right)=F_{j}(j=0,1,2)$.

(ii) We say that $(\mathscr{E}, \Pi, p)$ is $\operatorname{good}$ if $(\rho, \delta) \neq 0$ or if $(\mathscr{E}, \Pi, p)$ is $i$-good for some $i$ with $1 \leq i \leq N$.

Proposition 3.2.2. Any $(\mathscr{E}, \Pi, p) \in \mathrm{CD}(N)$ is good.

Proof. This can be checked directly.

Q. E. D.

In case $N \geq 4,(\mathscr{E}, \Pi, p) \in \mathrm{CD}(N)$ is $i$-good for any $i$ with $1 \leq i \leq N$.

Example 3.2.3. Let $(\mathscr{E}, \Pi, p) \in \mathrm{CD}(3)$ be such that the corresponding Dynkin diagram is 


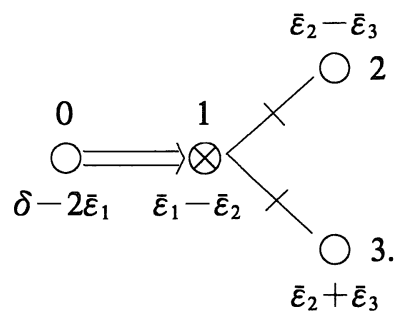

\section{Moreover}

$$
\begin{aligned}
& \Phi(\mathscr{E}, \Pi, p) \cap P_{+} \\
& =\left\{x+s \delta,-x+(s+1) \delta(s \geq 0) \mid x=\bar{\varepsilon}_{1}-\bar{\varepsilon}_{2}, \bar{\varepsilon}_{2}-\bar{\varepsilon}_{3}, \bar{\varepsilon}_{2}+\bar{\varepsilon}_{3},\right. \\
& \left.\quad \bar{\varepsilon}_{1}-\bar{\varepsilon}_{3}, \bar{\varepsilon}_{1}+\bar{\varepsilon}_{3}, \bar{\varepsilon}_{1}+\bar{\varepsilon}_{2}, 2 \bar{\varepsilon}_{1}\right\} .
\end{aligned}
$$

Then $(\mathscr{E}, \Pi, p)$ is not 1-good since the Dynkin diagram of $\Pi_{[1]}=\left\{\delta-\theta_{1}-\theta_{2}, \theta_{1}-\right.$ $\left.\theta_{2}, \theta_{1}+\theta_{2}\right\}$ is not connected. On the other hand $\Pi_{[2]}=\left\{\delta-2 \theta_{1}, \theta_{1}-\theta_{2}, \theta_{1}+\theta_{2}\right\}$, and the diagram of $\left(\mathscr{E}_{[2]}, \Pi_{[2]}, p_{[2]}\right)$ is:

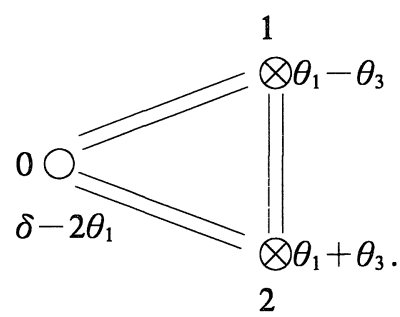

Hence $(\mathscr{E}, \Pi, p)$ is 2 -good. Similary we see that it is also 3 -good.

3.3. Keep the notation as above. Assume $(\mathscr{E}, \Pi, p) \in \mathrm{CD}(N)$ to be an $i$-good datum. Let $\Phi(\mathscr{E}, \Pi, p)_{[i]}=\Phi(\mathscr{E}, \Pi, p) \cap \mathscr{E}_{[i]}$. Let $x_{0}, \ldots, x_{N-1}$ be

$$
\begin{cases}s_{0}, \ldots, s_{i-2}, s_{i} s_{i-1} s_{i}, s_{i+1}, \ldots, s_{N} & \text { if } 1 \leq i \leq N-1, \\ s_{0}, \ldots, s_{N-2}, s_{N-1} s_{N} s_{N-1} & \text { if } i=N\end{cases}
$$

respectively. Then the following diagram commutes:

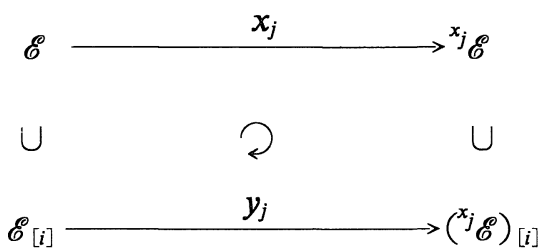


where $y_{j}$ is defined in the same manner as $s_{j}$ in Proposition 2.4.3 with $\theta_{r}$ in place of $\bar{\varepsilon}_{r}(1 \leq r \leq N-1)$. By the same argument as in the proof of Proposition 2.6.1, we see that, if $\gamma \in \Phi(\mathscr{E}, \Pi, p)_{[i]} \backslash \mathbf{Z} \delta$, then there exists a composition $w=x_{i(k)} \cdots x_{i(2)} x_{i(1)}$ such that $w(\gamma) \in \mathbf{Z}\left({ }^{w} \Pi\right)_{[i]} \cap \Phi\left({ }^{w} \mathscr{E},{ }^{w} \Pi,{ }^{w} p\right)_{[i]}$. It is easily verified that $w(\gamma) \in$ $\Phi\left(\left({ }^{w} \mathscr{E}\right)_{[i]},\left({ }^{w} \Pi\right)_{[i]},\left({ }^{w} p\right)_{[i]}\right)$. Applying $y_{i(1)} y_{i(2)} \cdots y_{i(k)}$, we see $\gamma \in \Phi\left(\mathscr{E}_{[i]}, \Pi_{[i]}, p_{[i]}\right)$ $\backslash \mathbf{Z} \delta$. Thus we conclude:

Proposition 3.3.1. Let $\mathscr{G}^{\natural}(\mathscr{E}, \Pi, p)$ be as in Definition 3.1.2. Assume ( $\mathscr{E}, \Pi$, $p) \in \operatorname{CD}(N)$ to be i-good. Then

$$
\Phi\left(\mathscr{E}_{[i]}, \Pi_{[i]}, p_{[i]}\right) \backslash \mathbf{Z} \delta=\Phi(\mathscr{E}, \Pi, p)_{[i]} \backslash \mathbf{Z} \delta
$$

Moreover there exists a homomorphism $j_{[i]}: \mathscr{G}^{\natural}\left(\mathscr{E}_{[i]}, \Pi_{[i]}, p_{[i]}\right) \rightarrow \mathscr{G}^{\natural}(\mathscr{E}, \Pi, p)$ such that $j_{[i]}\left(H_{\nu}\right)=H_{\nu}$. In particular, $j_{[i]}\left(\mathscr{G}^{\natural}\left(\mathscr{E}_{[i]}, \Pi_{[i]}, p_{[i]}\right)_{\beta}\right)=\mathscr{G}^{\natural}(\mathscr{E}, \Pi, p)_{\beta}$ for $\beta \in$ $\Phi\left(\mathscr{E}_{[i]}, \Pi_{[i]}, p_{[i]}\right) \backslash \mathbf{Z} \delta$ (see also Proposition 3.1.1) (If $\left(\mathscr{E}_{[i]}, \Pi_{[i]}, p_{[i]}\right)$ is of type (ia) or (ic) of Definition 3.2.1, we define $\mathscr{G}^{\natural}\left(\mathscr{E}_{[i]}, \Pi_{[i]}, p_{[i]}\right)=\mathscr{G}\left(\mathscr{E}_{[i]}, \Pi_{[i]}, p_{[i]}\right)$.)

3.4. Keep the notation in 1.5. The notation $(\mathrm{XY})_{i}^{b}$ means $(\mathrm{XY})_{i}$ with $\sum_{i=1}^{N} \bar{d}_{i}=$ 0 . For $(\mathscr{E}, \Pi, p) \in \mathrm{CD}(N)$, define $\mathscr{G}^{\natural \natural}(\mathscr{E}, \Pi, p) \in A I(\mathscr{E}, \Pi, p)$ as follows:

(a) If $(\mathscr{E}, \Pi, p)$ is (CC $)_{1}^{b},(\mathrm{CD})_{0}^{b},(\mathrm{DD})_{1}^{b}$ or (DC) ${ }_{0}^{b}$, then we let $\mathscr{G}^{\natural \natural}(\mathscr{E}, \Pi$, $p)$ be the affine-admissible Lie superalgebra isomorphic to $s l(N, N)^{(2)}$.

(b) Otherwise we put $\mathscr{G}^{\natural}(\mathscr{E}, \Pi, p)=\mathscr{G}(\mathscr{E}, \Pi, p)$.

Proposition 3.4.1. If $(\mathscr{E}, \Pi, p) \in \mathrm{CD}(N)$, then $\mathscr{G}^{\natural}(\mathscr{E}, \Pi, p)=\mathscr{G}^{\natural}(\mathscr{E}, \Pi, p)$.

Proof. It suffices to show

$$
\operatorname{dim} \mathscr{G}_{s \delta}^{\natural}=\operatorname{dim} \mathscr{G}_{s \delta}^{\natural} \text { for any } s .
$$

If $(\delta, \rho) \neq 0$, this is an immediate consequence of Proposition 3.1.3. So we assume $(\delta, \rho)=0$.

We shall prove the theorem for $\mathscr{G}^{\natural}=\mathscr{G}^{\natural}(\mathscr{E}, \Pi, p)$ such that the corresponding Dynkin diagram is (CD). (Other cases can be treated similarly.) We use induction on $N$, and start with $N=3$. The unique datum of (CD) with $N=3$ and $(\delta, \rho)=0$ is given in Example 3.2.3. So we first assume $(\mathscr{E}, \Pi, p)$ to be this one. Hence $\mathscr{G}^{\text {ah }}$ $\cong \mathbf{D}(2,1)^{(1)}$ and $\operatorname{dim} \mathscr{G}_{s \delta}^{\natural}=3$ for $s \neq 0$. By Proposition 3.3.1, there is the homomorphism $j_{[2]}: \mathscr{G}^{\natural}\left(\mathscr{E}_{[2]}, \Pi_{[2]}, p_{[2]}\right) \rightarrow \mathscr{G}^{\natural}$. Let $\mathscr{G}_{[2]}^{\natural}$ be the Lie sub-superalgebra of $\mathscr{G}^{\natural}$ generated by the elements

$$
H \in \mathscr{H}_{[2]}, E_{0},\left[E_{1}, E_{2}\right],\left[E_{1}, E_{3}\right], F_{0},-\bar{d}_{2}\left[F_{1}, F_{2}\right],-\bar{d}_{2}\left[F_{1}, F_{3}\right]
$$


Since $\mathscr{G}_{[2]}^{\natural}=j_{[2]}\left(\mathscr{G}^{\natural}\left(\mathscr{E}_{[2]}, \Pi_{[2]}, p_{[2]}\right)\right)$, by Proposition $3.3 .1, \mathscr{G}_{[2]}^{\natural}$ is also affineadmissible with respect to $\left(\mathscr{E}_{[2]}, \Pi_{[2]}, p\right)$ and the generators (3.4.2). However, since $\left(\mathscr{E}_{[2]}, \Pi_{[2]}, p_{[2]}\right)$ satisfies $(\delta, \rho) \neq 0$, by Proposition 3.1 .3 , we have

$$
\mathscr{G}_{[2]}^{\natural} \cong \mathscr{G}\left(\mathscr{E}_{[2]}, \Pi_{[2]}, p_{[2]}\right) \cong \mathrm{A}(1,0)^{(1)} .
$$

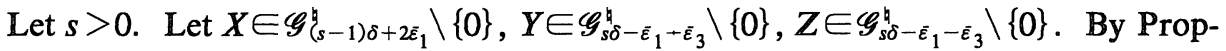

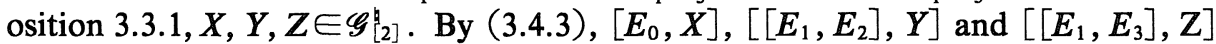
span the 2-dimensional space $\left(\mathscr{G}_{[2]}^{\natural}\right)_{s \delta}$. On the other hand, $\mathscr{G}_{s \delta}^{\natural}$ is spanned by $\left[E_{0}, X\right],\left[E_{1},\left[E_{2}, Y\right]\right],\left[E_{2},\left[E_{1}, Y\right]\right]$ and $\left[E_{3},\left[E_{1}, Z\right]\right]$. Since $\left[E_{2},\left[E_{1}, Y\right]\right]=$ $\left[E_{1},\left[E_{2}, Y\right]\right]-\left[\left[E_{1}, E_{2}\right], Y\right],\left[E_{3},\left[E_{1}, Z\right]\right]=\left[E_{1},\left[E_{3}, Z\right]\right]-\left[\left[E_{1}, E_{3}\right], Z\right]$ and $\left[E_{3}, Z\right]=a\left[E_{2}, Y\right]$ for some $a \in C, \mathscr{G}_{s \delta}^{\natural}$ is spanned by $\left(\mathscr{G}_{[2]}^{\natural}\right)_{s \delta}$ and $\left[E_{1},\left[E_{2}, Y\right]\right]$. Hence $\operatorname{dim} \mathscr{G}_{s \delta}^{\natural}=3$. Similarly we can show $\operatorname{dim} \mathscr{G}_{-s \delta}^{\natural}=3$. This completes the proof for (CD) with $N=3$.

Similar argument applies to (CD) with $N \geq 4$. In this case, we can prove (3.4.1) using the subalgebra $\mathscr{G}_{[2]}^{\natural}$ of $\mathscr{G}^{\natural}$ generated by the elements

$$
H \in \mathscr{H}_{[2]}, E_{0},\left[E_{1}, E_{2}\right], E_{j}(3 \leq j \leq N), F_{0},-\bar{d}_{2}\left[F_{1}, F_{2}\right], F_{j}(3 \leq j \leq N) .
$$

3.5. By the same argument as in the proof of Proposition 3.4.1, we have

Theorem 3.5.1. Let $(\mathscr{E}, \Pi, p) \in \operatorname{ABCD}(N), \mathscr{G}=\mathscr{G}(\mathscr{E}, \Pi, p)$ and $\mathscr{G}^{\natural}=\mathscr{G}^{\natural}(\mathscr{E}$, $\Pi, p)$. Then we have:

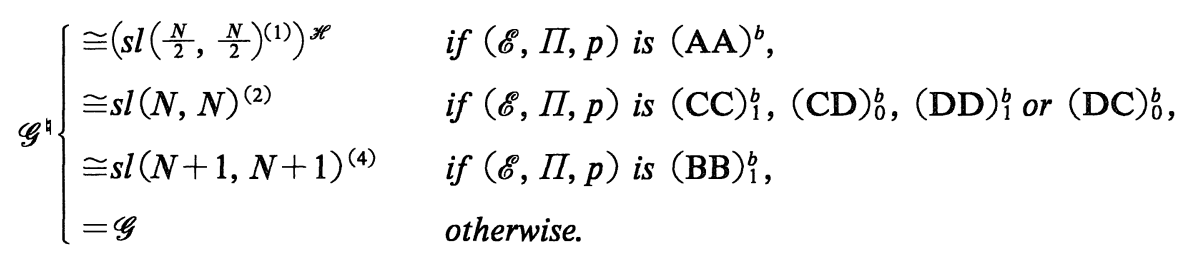

In particular,

$$
\operatorname{dim}\left(\operatorname{ker} \Psi\left[\mathscr{G}^{\natural}, \mathscr{G}\right] \cap \mathscr{G}_{r \delta}^{\natural}\right)= \begin{cases}1 & \text { if }(\mathscr{E}, \Pi, p) \text { is }(\mathrm{AA})^{b} \text { and } r \in \mathbf{Z} \backslash\{0\}, \\ 1 & \text { if }(\mathscr{E}, \Pi, p) \text { is }(\mathrm{CC})_{1}^{b},(\mathrm{CD})_{0}^{b},(\mathrm{DD})_{1}^{b} \text { or } \\ & (\mathrm{DC})_{0}^{b}, \text { and } r \in 2 \mathbf{Z}+1, \\ 1 & \text { if }(\mathscr{E}, \Pi, p) \text { is }(\mathrm{BB})_{1}^{b} \text { and } r \in 4 \mathbb{Z}+2, \\ 0 & \text { otherwise. }\end{cases}
$$

See also Example 3.1.4. 


\section{§4. Relations for Algebras of Affine ABCD-Types}

4.1.

Theorem 4.1.1. Let $(\mathscr{E}, \Pi, p)$ be of affine $\mathrm{ABCD}$ type. Then the Lie superalgebra $\mathscr{G}^{\natural}(\mathscr{E}, \Pi, p)$ can also be defined by generators $H \in \mathscr{H}, E_{i}, F_{i}(0 \leq i \leq n)$, parities $p(H)=0, p\left(E_{i}\right)=p\left(E_{i}\right)=p\left(\alpha_{i}\right)$ and relations:

$$
\left[H, H^{\prime}\right]=0, \quad\left(H, H^{\prime} \in \mathscr{H}\right)
$$

$$
\left[\boldsymbol{H}, \boldsymbol{E}_{i}\right]=\alpha_{i}(\boldsymbol{H}) \boldsymbol{E}_{i}, \quad\left[\boldsymbol{H}, \boldsymbol{F}_{i}\right]=-\alpha_{i}(\boldsymbol{H}) \boldsymbol{F}_{i},
$$

(S4) (1) $\left[E_{i}, E_{j}\right]=0$ if $\left(\alpha_{i}, \alpha_{j}\right)=0(i \neq j)$,

(S4)(2) $\left[E_{i}, E_{i}\right]=0$

(S4) (3) $\left[E_{i},\left[E_{i}, \ldots,\left[E_{i}, E_{j}\right] \ldots\right]\right]=0\left(E_{i}\right.$ appears $1-\frac{2\left(\alpha_{i}, \alpha_{j}\right)}{\left(\alpha_{i}, \alpha_{i}\right)}$ times $)$

$$
\text { if }\left(\alpha_{i}, \alpha_{i}\right) \neq 0 \text { and }(-1)^{\left\{p\left(\alpha_{i}\right) \frac{2\left(\alpha_{i}, \alpha_{i}\right)}{\left(\alpha_{i}, \alpha_{i}\right)}\right\}}=1 \text {, }
$$

(S4) (4) $\quad\left[\left[\left[E_{i}, E_{j}\right], E_{k}\right], E_{J}\right]=0$

if $\quad \stackrel{i}{\times} \stackrel{-x}{-j} \stackrel{x}{\otimes}^{k} \times(x \neq 0)$,

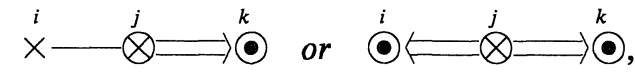

(S4)(5) $\left[\left[\left[E_{i}, E_{j}\right],\left[\left[E_{i}, E_{j}\right], E_{k}\right]\right], E_{j}\right]=0$

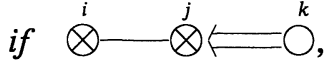

(S4) (6) $\quad\left[\left[\left[\left[\left[\left[E_{i}, E_{j}\right], E_{k}\right], E_{l}\right], E_{k}\right], E_{j}\right], E_{k}\right]=0$

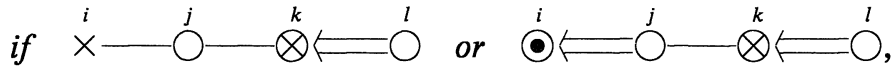

$(\mathrm{S} 4)(7) \quad(-1)^{p\left(\alpha_{i}\right) p\left(\alpha_{k}\right)}\left(\alpha_{i}, \alpha_{k}\right)\left[\left[E_{i}, E_{j}\right], E_{k}\right]=(-1)^{p\left(\alpha_{i}\right) p\left(\alpha_{j}\right)}\left(\alpha_{i}, \alpha_{j}\right)\left[\left[E_{i}, E_{k}\right], E_{j}\right]$

$$
\begin{array}{r}
\text { if }\left(\alpha_{i}, \alpha_{j}\right) \neq 0,\left(\alpha_{j}, \alpha_{k}\right) \neq 0,\left(\alpha_{k}, \alpha_{i}\right) \neq 0, \\
\left(\alpha_{i}, \alpha_{j}\right)+\left(\alpha_{j}, \alpha_{k}\right)+\left(\alpha_{k}, \alpha_{i}\right)=0 \\
\text { and } p\left(\alpha_{i}\right) p\left(\alpha_{j}\right)+p\left(\alpha_{j}\right) p\left(\alpha_{k}\right)+p\left(\alpha_{k}\right) p\left(\alpha_{i}\right)=\overline{1},
\end{array}
$$

$(\mathrm{S} 4)(8) \quad\left[\left[\left[E_{j}, E_{i}\right],\left[E_{j}, E_{k}\right]\right],\left[E_{j}, E_{l}\right]\right]=\left[\left[\left[E_{j}, E_{i}\right],\left[E_{j}, E_{l}\right]\right],\left[E_{j}, E_{k}\right]\right]$

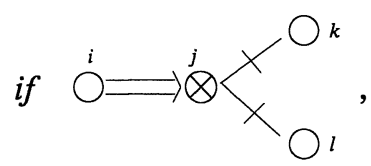

$(\mathrm{S} 4)(9) \quad\left[\left[\left[E_{k},\left[E_{l},\left[E_{k}, E_{j}\right]\right]\right],\left[E_{k},\left[E_{l},\left[E_{k},\left[E_{j}, E_{i}\right]\right]\right]\right]\right], E_{j}\right]$ 
$=2\left[\left[E_{k}, E_{j}\right],\left[\left[E_{l},\left[E_{k}, E_{j}\right]\right],\left[E_{k},\left[E_{l},\left[E_{k},\left[E_{j}, E_{i}\right]\right]\right]\right]\right]\right]$

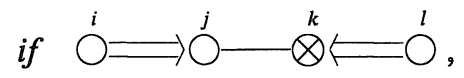

(S4) (10) $\left[E_{j},\left[E_{k},\left[E_{j},\left[E_{k}, E_{i}\right]\right]\right]\right]=\left[E_{k},\left[E_{j},\left[E_{k},\left[E_{j}, E_{i}\right]\right]\right]\right]$

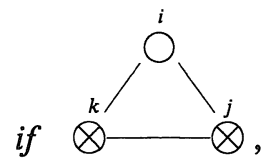

(S4) (11) $\left[\left[E_{k}, E_{j}\right],\left[\left[E_{k}, E_{j}\right],\left[\left[E_{k}, E_{j}\right], E_{i}\right]\right]\right]$

$$
\begin{aligned}
& =\left(1-(-1)^{p\left(\alpha_{k}\right)} 2\right)\left[\left[\left[E_{k}, E_{j}\right],\left[E_{k},\left[E_{k},\left[E_{j}, E_{i}\right]\right]\right]\right], E_{j}\right] \\
& \text { if } \stackrel{\imath}{\bigcirc} \Longrightarrow \stackrel{j}{=}
\end{aligned}
$$

(S5) (a) $(1 \leq a \leq 11)$ The same relations with $F_{r}$ 's in place of $E_{r}$ 's in (S4) (a).

Proof. Let $\mathscr{G}^{b}=\mathscr{G}^{b}(\mathscr{E}, \Pi, p)$ be the Lie superalgebra defined with the generators $H_{\lambda}(\lambda \in \mathscr{E}), E_{m}, F_{m}(0 \leq m \leq n=|\Pi|-1)$ and the defining relations (S1), (S2), (S3), (S4) (a), (S5) (a) $(a=1, \ldots, 11)$. Let $x(a)$ (resp. $y(a))$ denote the left hand side minus the right hand side of the equality in (S4) (a) (resp. (S5) (a)). If a finite sequence $\alpha_{u}, \alpha_{v}, \ldots, \alpha_{w}$ of simple roots satisfies the assumption in (S4) (a) (resp. (S5) (a)) with $u, v, \ldots, w$ in place of $i, j, \ldots, k$, then let $x(a)_{u v \ldots w}^{i j \ldots k}$ (resp. $\left.y(a)_{u v \ldots w}^{i j \ldots k}\right)$ denote the element of $\tilde{\mathscr{G}}(\mathscr{E}, \Pi, p)$ obtained by substituting $E_{u}, E_{v}$, $\ldots, E_{w}$ (resp. $F_{u}, F_{v}, \ldots, F_{w}$ ) for $E_{i}, E_{j}, \ldots, E_{k}$ (resp. $F_{i}, F_{j}, \ldots, F_{k}$ ) in the definition of $x(a)$ (resp. $y(a))$. Let $X(\mathscr{E}, \Pi, p)$ (resp. $Y(\mathscr{E}, \Pi, p)$ ) denote the set of all the $x(a)_{u v \ldots w}^{i j . \ldots k}$ (resp. $\left.y(a)_{u v \ldots w}^{i j . \ldots k}\right)$. See 4.4 (example). Let $r^{b}$ be the ideal of $\tilde{\mathscr{G}}=\tilde{\mathscr{G}}(\mathscr{E}, \Pi$, p) generated by the elements of $X(\mathscr{E}, \Pi, p) \cup Y(\mathscr{E}, \Pi, p)$. From now on, we identify $\mathscr{G}^{b}$ with $\tilde{\mathscr{G}} / r^{b}$.

Step 1. $\mathscr{G}^{b}(\mathscr{E}, \Pi, p)$ is admissible. Keep the notation in 1.2. Let $r_{+}^{b}$ (resp. $r_{-}^{b}$ ) be the ideal of $\tilde{N}^{+}$(resp. $\tilde{N}^{-}$) generated by the elements of $X(\mathscr{E}, \Pi, p)$ (resp. $Y(\mathscr{E}, \Pi, p))$. By direct calculation, whose example will be given in 4.3 , we get

$$
\left[r_{+}^{b}, F_{m}\right] \subset r_{+}^{b} \quad \text { for any } 0 \leq m \leq n=|\Pi|-1 \text {. }
$$

Let $\xi=\tilde{\xi}(\mathscr{E}, \Pi, p): \tilde{\mathscr{G}}(\mathscr{E}, \Pi, p) \rightarrow \tilde{\mathscr{G}}(\mathscr{E}, \Pi, p)$ be an isomorphism defined by $\tilde{\xi}(H)=$ $-H, \tilde{\xi}\left(E_{i}\right)=F_{i}, \tilde{\xi}\left(F_{i}\right)=(-1)^{p\left(\alpha_{i}\right)} E_{i}$. Applying $\xi$ to (4.1.1), we have [rb $\left.\left.\boldsymbol{r}_{-}^{\mathrm{b}}\right] \subset \boldsymbol{E}_{m}\right]$ for any $m$. Hence $r^{b}=r_{+}^{b} \oplus r_{-}^{b}$, and $r^{b}$ is an admissible ideal (see Definition 1.2.1), which implies that $\mathscr{G}^{b}(\mathscr{E}, \Pi, p)$ is admissible.

Step 2. Each $L_{i}$ lift where $L_{i}$ is of Theorem 2.5.1. By the result of Step 1, 
there is the epimorphism $\Psi^{b}(\mathscr{E}, \Pi, p)=\Psi\left[\mathscr{G}^{b}(\mathscr{E}, \Pi, p), \mathscr{G}(\mathscr{E}, \Pi, p)\right]: \mathscr{G}^{b}(\mathscr{E}, \Pi, p)$ $\rightarrow \mathscr{G}(\mathscr{E}, \Pi, p)$. Then the assertion of Step 2 means that, for any $(\mathscr{E}, \Pi, p)$ of affine ABCD-type, there is an homomorphism $L_{i}^{b}=L_{i}^{b}(\mathscr{E}, \Pi, p): \mathscr{G}^{b}(\mathscr{E}, \Pi, p) \rightarrow \mathscr{G}^{b}\left({ }^{s_{i}} \mathscr{E}\right.$, $\left.{ }^{s_{i}} \Pi,{ }^{s_{i}} p\right)$ such that the following diagram commutes:

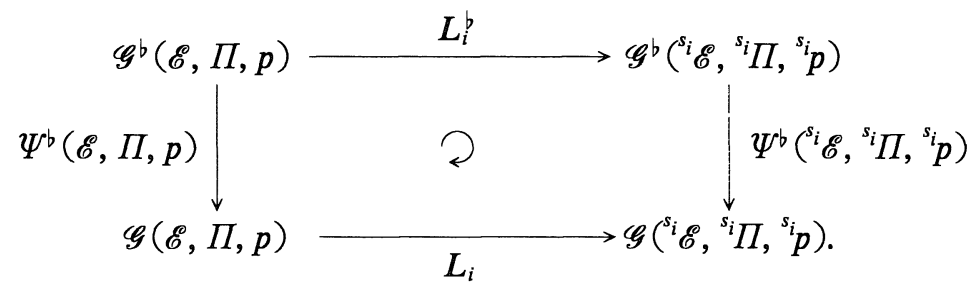

We note that $L_{i}^{b}$ is an isomorphism since $L_{i}^{b}\left({ }^{s_{i}} \mathscr{E},{ }^{s_{i}} \Pi,{ }^{s_{i}} p\right) \circ L_{i}^{b}(\mathscr{E}, \Pi, p) \equiv i d_{\mathscr{G} b(\delta, \Pi, p)}$ and $\left.L_{i}^{b}(\mathscr{E}, \Pi, p) \circ L_{i}^{b}\left({ }^{s_{i}} \mathscr{E},{ }^{s_{i}} \Pi,{ }^{s_{i}} p\right) \equiv i d_{\mathscr{G} b} s_{i_{\delta_{\delta}},}, s_{i_{\Pi},} s_{i p}\right)$ in the sense of Definition 1.2.5.

If $s_{i}$ is a reflection, then $L_{i}$ lift to $L_{i}^{\text {b }}$ by Proposition 2.2.7. If $s_{i}$ is a diagram automorphism, it is clear that $L_{i}^{b}$ exists. In case $s_{i}$ is a super-reflection, the existence of $L_{i}^{b}$ is shown as follows. We also denote $L_{i}$ and $L_{i}^{b}$ by $L_{i}(\mathscr{E}, \Pi, p)$ and $L_{i}^{b}(\mathscr{E}, \Pi$, $p$ ) to clarify their domains of definition. Using formulas of Lemma 2.2.1, it is easily verified that there exists an homomorphism $L_{i}^{M b}(\mathscr{E}, \Pi, p): \tilde{\mathscr{G}}(\mathscr{E}, \Pi, p) \rightarrow \mathscr{G}^{b}\left({ }^{s_{i}} \mathscr{E},{ }^{s_{i}} \Pi\right.$, $\left.s_{i} p\right)$ such that $L_{i}^{M b}(\mathscr{E}, \Pi, p)$ satisfies the same equalities as $(2.2 .10-11)$ with $L_{i}^{M b}(\mathscr{E}$, $\Pi, p)$ in place of $\phi$. To show that $L_{i}^{b}(\mathscr{E}, \Pi, p)$ exists is exactly to show that

$$
L_{i}^{M b}(\mathscr{E}, \Pi, p)\left(x(a)_{u v \ldots w}^{i j \ldots k}\right)=0 \quad \text { for any } x(a)_{u v \ldots w}^{i j \ldots . k} \in X(\mathscr{E}, \Pi, p)
$$

and

$$
L_{i}^{M b}(\mathscr{E}, \Pi, p)\left(y(a)_{u v \ldots w}^{i j \ldots k}\right)=0 \quad \text { for any } y(a)_{u v \ldots w}^{i j \ldots k} \in Y(\mathscr{E}, \Pi, p)
$$

Since $\tilde{\xi}\left(r^{b}\right)=r^{b}, \xi$ induces an isomorphism $\xi^{b}=\xi^{b}(\mathscr{E}, \Pi, p): \mathscr{G}^{b}(\mathscr{E}, \Pi, p) \rightarrow$ $\mathscr{G}^{b}(\mathscr{E}, \Pi, p)$. Since $L_{i}^{M b}(\mathscr{E}, \Pi, p) \circ \tilde{\xi}(\mathscr{E}, \Pi, p) \equiv \xi^{b}\left({ }^{s_{i}} \mathscr{E},{ }^{s_{i}} \Pi,{ }^{s_{i}} p\right) \circ L_{i}^{M b}\left({ }^{s_{i}} \mathscr{E},{ }^{s_{i}} \Pi,{ }^{s_{i}} p\right)$ (see Definition 1.2.5) and $\tilde{\xi}\left(y(a)_{u v \ldots .}^{i j \ldots k}\right)= \pm x(a)_{u v \ldots w}^{i j \ldots k}$, we only need to show (4.1.2). The statement (4.1.2) is verified by direct calculation whose example will be given in 4.4 .

Step 3. $\mathscr{G}^{b}(\mathscr{E}, \Pi, p)$ is affine-admissible. Keep the notation in 2.6. Let $\alpha$ $\in\left(\boldsymbol{P}_{+} \cup \boldsymbol{P}_{-}\right) \backslash \mathbf{Z} \delta$, and let $\boldsymbol{w}$ be the element given in the statement of Proposition 2.6.1. If $w$ is written as $w=s_{i(1)} s_{i(2)} \cdots s_{i(k)}$, put $L_{w}^{b}=L_{i(1)}^{b} \circ L_{i(2)}^{b} \circ \cdots \circ L_{i(r)}^{b}$. Then $L_{w}^{b}: \mathscr{G}^{b}(\mathscr{E}, \Pi, p) \rightarrow \mathscr{G}^{b}\left({ }^{w} \mathscr{E},{ }^{w} \Pi,{ }^{w} p\right)$ is an isomorphism such that $L_{w}^{b}\left(\mathscr{G}^{b}(\mathscr{E}, \Pi, p)_{\gamma}\right)=$ $\mathscr{G}^{b}\left({ }^{w} \mathscr{E},{ }^{w} \Pi,{ }^{w} p\right)_{w(r)}$. (Isomorphisms denoted by $L_{w}^{b}$ are equivalent each other in the sense of Definition 1.2.5.) By Proposition 2.6.1 and Lemma 1.2.3, we have 


$$
\begin{aligned}
\operatorname{dim} \mathscr{G}_{\alpha}^{b}=\operatorname{dim} L_{w}^{b}\left(\mathscr{G}_{\alpha}^{b}\right) & =\operatorname{dim} \mathscr{G}^{b}\left({ }^{w} \mathscr{E},{ }^{w} \Pi,{ }^{w} p\right)_{w(\alpha)} \\
& = \begin{cases}1 & \text { if } \alpha \in \Phi(\mathscr{E}, \Pi, p) \backslash \mathbb{Z} \delta, \\
0 & \text { if } \alpha \in P \backslash \Phi(\mathscr{E}, \Pi, p),\end{cases}
\end{aligned}
$$

as desired.

Step 4. $\mathscr{G}^{b}(\mathscr{E}, \Pi, p)$ coincides with $\mathscr{G}^{\natural}(\mathscr{E}, \Pi, p)$. Keep the notation in 3.1 . Here we abbreviate $\mathscr{G}(\mathscr{E}, \Pi, p), \mathscr{G}^{b}(\mathscr{E}, \Pi, p), \mathscr{G}^{\natural}(\mathscr{E}, \Pi, p)$ to $\tilde{\mathscr{G}}, \mathscr{G}^{b}, \mathscr{G}^{\natural}$ respectively. By the result of Step 3, there is the epimorphism $\Psi^{\natural b}=\Psi\left[\mathscr{G}^{\natural}, \mathscr{G}^{b}\right]$. For $x(a)_{u v \ldots w}^{i j \ldots k} \in$ $X(\mathscr{E}, \Pi, p)$, let $\alpha_{u v \ldots w}=\alpha_{u}+\alpha_{v}+\cdots+\alpha_{w} \in P_{+}$. Then $x(a)_{u v \ldots w}^{i j \ldots k} \in \tilde{\mathscr{G}}(\mathscr{E}, \Pi, p)_{\alpha_{u v \ldots w}}$. Let $\left(x(a)_{u v \ldots w}^{i j \ldots k}\right)^{\natural}=\Psi\left[\tilde{\mathscr{G}}, \mathscr{G}^{\natural}\right]\left(x(a)_{u v \ldots w}^{i j \ldots k}\right) \in \mathscr{G}^{\natural}(\mathscr{E}, \Pi, p)$. Since $\alpha_{u v \ldots w} \not \subset \mathbf{Z} \delta, \Psi_{\mid \mathscr{S}_{\alpha_{u v \ldots w}}^{\natural b}}^{i v \ldots w}$ is

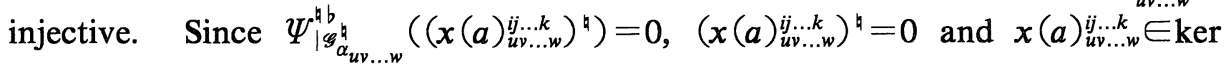
$\Psi\left[\tilde{\mathscr{G}}, \mathscr{G}^{\natural}\right]$. Similarly we have $y(a)_{u v \ldots w}^{i j . \ldots k} \in \operatorname{ker} \Psi\left[\tilde{\mathscr{G}}, \mathscr{G}^{\natural}\right]$ for $y(a)_{u v \ldots w}^{i j \ldots k} \in Y(\mathscr{E}, \Pi, p)$. Hence there is the epimorphism $\Psi^{b \natural}=\Psi\left[\mathscr{G}^{b}, \mathscr{G}^{\natural}\right]$. It is clear that $\Psi^{b \natural} \circ \Psi^{\natural b}=1_{\mathscr{G}^{\natural}}$ and $\Psi^{\natural b} \circ \Psi^{b \natural}=1_{g b b}$, as desired.

\section{2.}

In 4.3-5.2, $L H S \sim R H S$ means $L H S=a \cdot R H S$ for some $a \in \mathbb{C}^{\times}$.

We note that the statement (S4) (7) does not depend on the order of $\{i, j, k\}$, namely,

$$
x(7)_{g(i) g(j) g(k)}^{i j k} \sim x(7)_{i j k}^{i j k} \quad \text { for any bijective map } g:\{i, j, k\} \rightarrow\{i, j, k\} .
$$

4.3. Here we shall show how to get (4.1.1) in the case that $(\mathscr{E}, \Pi, p)$ is $(\mathrm{XC})$ with $N \geq 4$. To do this, we show, by direct calculation, that

$$
\left[x(a) \underset{u v \ldots w}{i j \ldots k}, F_{m}\right] \in r_{+}^{b}
$$

for any $0 \leq m \leq N$ and any $x(a)_{u v \ldots . . .}^{i j . . k} \in X(\mathscr{E}, \Pi, p)$. Here, as an example of the direct calculation, we are going to show $\left[x(6)_{N-3, N-2, N-1, N}^{i j k l}, F_{m}\right] \in r_{+}^{b}$. By abuse of notation, we also denote $N-3, N-2, N-1, N$ by $i, j, k, l$. If $m \neq i, j, k, l$, it is clear that $\left[x(6)_{i j k l}^{i j k l}, F_{m}\right]=0$. We have:

$$
\begin{aligned}
& {\left[x(6)_{i j k l}^{i j k l}, F_{i}\right]+r_{+}^{b}} \\
& \quad\left[\left[\left[\left[\left[\left[H_{i}, E_{j}\right], E_{k}\right], E_{l}\right], E_{k}\right], E_{j}\right], E_{k}\right]+r_{+}^{b} \\
& \left.\sim\left[\left[\left[\left[E_{j}, E_{k}\right], E_{l}\right],\left[E_{k}, E_{j}\right]\right], E_{k}\right]+r_{+}^{b} \quad \text { (by using } x(1)_{j l}^{i j} \text { and } x(3)_{j j k}^{i j}\right) \\
& \sim 0+r_{+}^{b} \quad \text { (by Lemma 2.2.1(iii)), }
\end{aligned}
$$


$\left[x(6)_{i j k l}^{i j k l}, F_{j}\right]+r_{+}^{b}$

$\sim \quad\left[\left[\left[\left[\left[\left[E_{i}, H_{j}\right], E_{k}\right], E_{l}\right], E_{k}\right], E_{j}\right], E_{k}\right]+\left[\left[\left[\left[\left[\left[E_{i}, E_{j}\right], E_{k}\right], E_{l}\right], E_{k}\right], H_{j}\right], E_{k}\right]+r_{+}^{b}$

$\sim 0+r_{+}^{b} \quad$ (by using $x(1)_{i k}^{i j}$ and $\left.x(2)_{k}^{i}\right)$,

$$
\begin{aligned}
{\left[x(6)_{i j k l}^{i j k l}, F_{k}\right]+r_{+}^{b} } \\
\quad\left[\left[\left[\left[\left[\left[E_{i}, E_{j}\right], E_{k}\right], E_{l}\right], E_{k}\right], E_{j}\right], H_{k}\right]-\left[\left[\left[\left[\left[\left[E_{i}, E_{j}\right], E_{k}\right], E_{l}\right], H_{k}\right], E_{j}\right], E_{k}\right] \\
\quad+\left[\left[\left[\left[\left[\left[E_{i}, E_{j}\right], H_{k}\right], E_{l}\right], E_{k}\right], E_{j}\right], E_{k}\right]+r_{+}^{b} \\
\sim \quad 0-0+0+r_{+}^{b}
\end{aligned}
$$

(by $\left(\alpha_{i}+\alpha_{j}+\alpha_{k}+\alpha_{l}+\alpha_{k}+\alpha_{j}, \alpha_{k}\right)=0$ for the 1 st term,

by using $x$ (3) $)_{j j k}^{i j}, x(3)_{j i j}^{i j i}$ and Lemma 2.2.1 (iii) for the 2nd term, and by using $x(1)_{j l}^{i j}$ for the 3 rd term)

and

$$
\begin{aligned}
& {\left[x(6)_{i j k l}^{i j k l}, F_{l}\right]+r_{+}^{b}} \\
& \left.\quad \sim\left[\left[\left[\left[\left[E_{i}, E_{j}\right], E_{k}\right], H_{l}\right], E_{k}\right], E_{j}\right], E_{k}\right]+r_{+}^{b} \\
& \left.\quad \sim 0+r_{+}^{b} \quad \text { (by using } x(2)_{k}^{i}\right) .
\end{aligned}
$$

So we got (4.3.1) in the case $a=6$. Other cases can be treated similarly. Once (4.3.1) is established, the statement (4.1.1) follows immediately.

4.4. Here we shall show how to get (4.1.2) in the case $(\mathscr{E}, \Pi, p)$ such that $\mathscr{G}(\mathscr{E}$, $\Pi, p) \cong \mathbf{A}(3,1)^{(2)}$. The Dynkin diagrams of $(\mathscr{E}, \Pi, p)$ 's change under superreflections as follows:

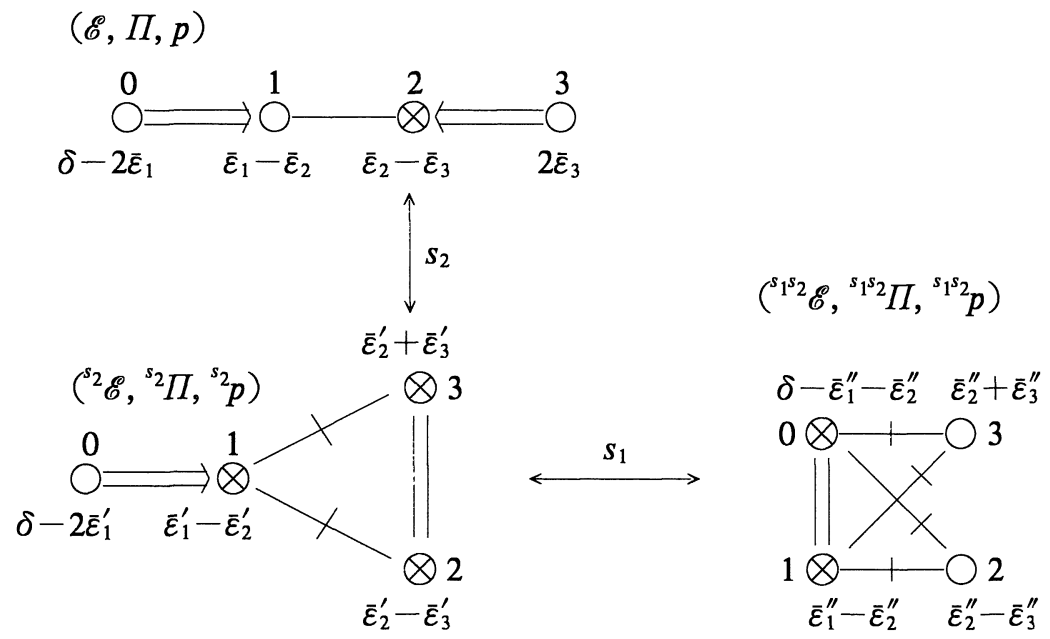


where $\bar{d}_{1}=\bar{d}_{2}=-\bar{d}_{3}=\bar{d}_{1}^{\prime}=-\bar{d}_{2}^{\prime}=\bar{d}_{3}^{\prime}=-\bar{d}_{1}^{\prime \prime}=\bar{d}_{2}^{\prime \prime}=\bar{d}_{3}^{\prime \prime}\left(\right.$ here $\bar{d}_{i}=\left(\bar{\varepsilon}_{i}, \bar{\varepsilon}_{i}\right), \bar{d}_{i}^{\prime}=\left(\bar{\varepsilon}_{i}^{\prime}, \bar{\varepsilon}_{i}\right)$ and $\left.\bar{d}_{i}^{\prime \prime}\left(\bar{\varepsilon}_{i}^{\prime \prime}, \bar{\varepsilon}_{i}^{\prime \prime}\right)\right)$. We note

$$
\begin{aligned}
X(\mathscr{E}, \Pi, p)= & \left\{x(1)_{02}^{i j}, x(1)_{03}^{i j}, x(1)_{13}^{i j}, x(2)_{2}^{i}, x(3)_{001}^{i j j}, x(3)_{1110}^{i i j j},\right. \\
& \left.x(3)_{112}^{i i j}, x(3)_{332}^{i j j}, x(9)_{0123}^{i j k}\right\} \\
X\left({ }^{s_{2}} \mathscr{E},{ }^{s_{2}} \Pi,{ }^{s_{2}} p\right)= & \left\{x(1)_{02}^{i j}, x(1)_{03}^{i j}, x(2)_{1}^{i}, x(2)_{2}^{i}, x(2)_{3}^{i}, x(3)_{001}^{i j j},\right. \\
& \left.x(5)_{210}^{i j k}, x(5)_{310}^{i j k}, x(7)_{123}^{i j k}\right\} \\
X\left({ }^{s_{1} s_{2}} \mathscr{E},{ }^{s_{1} s_{2}} \Pi,{ }^{s_{1} s_{2}} p\right)= & \left\{x(1)_{23}^{i j}, x(2)_{0,}^{i j}, x(2)_{1}^{i}, x(3)_{220}^{i i j}, x(3)_{221}^{i i j}, x(3)_{330}^{i i j},\right. \\
& \left.x(3)_{331}^{i i j}, x(7)_{201}^{i j k}, x(7)_{301}^{i j k}\right\}
\end{aligned}
$$

We abbreviate $L_{2}^{M b}(\mathscr{E}, \Pi, p), L_{2}^{M b}\left({ }^{s_{2}} \mathscr{E},{ }^{s_{2}} \Pi,{ }^{s_{2}} p\right), L_{1}^{M b}\left({ }^{s_{2}} \mathscr{E},{ }^{s_{2}} \Pi,{ }^{s_{2}} p\right), L_{1}^{M b}\left({ }^{s_{1} s_{2}} \mathscr{E}\right.$, $\left.{ }^{s_{1} s_{2}} \Pi,{ }^{s_{1} s_{2}} p\right)$ to $L_{2}^{M b},{ }^{M} L_{2}^{M b}, L_{1}^{M b}, " L_{1}^{M b}$ respectively. The elements $\Psi[\tilde{\mathscr{G}}(\mathscr{E}, \Pi, p)$, $\left.\mathscr{G}^{b}(\mathscr{E}, \Pi, p)\right]\left(x(a)_{u v \ldots . . .}^{i j \ldots k}\right), \Psi\left[\tilde{\mathscr{G}}(\mathscr{E}, \Pi, p), \mathscr{G}^{b}(\mathscr{E}, \Pi, p)\right]\left(y(a)_{u v \ldots . . .}^{i j . . k}\right) \in \mathscr{G}^{b}(\mathscr{E}, \Pi, p)$ will also be denoted by $x(a)_{u v \ldots w}^{i j \ldots k}$ and $y(a)_{u v \ldots . . .}^{i j \ldots k}$. Then we have

$$
\begin{aligned}
L_{2}^{M b}\left(x(1)_{13}^{i j}\right) \sim & {\left[\left[E_{2}, E_{1}\right],\left[E_{2}, E_{3}\right]\right] } \\
\sim & {\left[E_{2},\left(\left[\left[E_{1}, E_{2}\right], E_{3}\right]-\left[\left[E_{1}, E_{3}\right], E_{2}\right]\right)\right] } \\
\sim & {\left[E_{2}, x(7)_{123}^{i j k}\right]=0, } \\
L_{2}^{M b}\left(x(3)_{1110}^{i i i j}\right) \sim & {\left[\left[E_{2}, E_{1}\right],\left[\left[E_{2}, E_{1}\right],\left[\left[E_{2}, E_{1}\right], E_{0}\right]\right]\right.} \\
= & {\left[E_{2},\left[E_{1},\left[\left[E_{2}, E_{1}\right],\left[\left[E_{2}, E_{1}\right], E_{0}\right]\right]\right]\right]-\left[E_{1},\left[E_{2},\right.\right.} \\
& {\left.\left.\left[\left[E_{2}, E_{1}\right],\left[\left[E_{2}, E_{1}\right], E_{0}\right]\right]\right]\right] } \\
= & 0 \text { (by (S4)(5) and Lemma 2.2.1 (iii)), }
\end{aligned}
$$

$$
\begin{aligned}
& L_{2}^{M b}\left(x(9)_{0123}^{i j k l}\right) \\
&=\quad L_{2}^{M b}\left(\left[\left[\left[E_{2},\left[E_{3},\left[E_{2}, E_{1}\right]\right]\right],\left[E_{2},\left[E_{3},\left[E_{2},\left[E_{1}, E_{0}\right]\right]\right]\right], E_{1}\right]\right.\right. \\
&\left.-2\left[\left[E_{2}, E_{1}\right],\left[\left[E_{3},\left[E_{2}, E_{1}\right]\right],\left[E_{2},\left[E_{3},\left[E_{2},\left[E_{1}, E_{0}\right]\right]\right]\right]\right]\right]\right) \\
& \sim {\left[\left[\left[F_{2},\left[\left[E_{2}, E_{3}\right],\left[F_{2},\left[E_{2}, E_{1}\right]\right]\right]\right],\left[F_{2},\left[\left[E_{2}, E_{3}\right],\left[F_{2},\left[\left[E_{2}, E_{1}\right], E_{0}\right]\right]\right]\right],\right.\right.} \\
& {\left.\left[E_{2}, E_{1}\right]\right]-2\left[\left[F_{2},\left[E_{2}, E_{1}\right]\right],\left[\left[\left[E_{2}, E_{3}\right],\left[F_{2},\left[E_{2}, E_{1}\right]\right]\right],\left[F_{2},\left[\left[E_{2}, E_{3}\right],\right.\right.\right.\right.} \\
& {\left.\left.\left.\left.\left[F_{2},\left[\left[E_{2}, E_{1}\right], E_{0}\right]\right]\right]\right]\right]\right] } \\
& \sim {\left.\left[\left[\left[E_{3}, E_{1}\right],\left[E_{3},\left[E_{1}, E_{0}\right]\right]\right],\left[E_{2}, E_{1}\right]\right]+\left[E_{1},\left[\left[\left[E_{2}, E_{3}\right], E_{1}\right],\left[E_{3},\left[E_{1}, E_{0}\right]\right]\right]\right]\right] } \\
&= {\left.\left.\left[\left[\left[E_{3}, E_{1}\right],\left[\left[E_{3}, E_{1}\right], E_{0}\right]\right],\left[E_{2}, E_{1}\right]\right]+\left[E_{1},\left[\left[\left[E_{2}, E_{3}\right], E_{1}\right],\left[\left[E_{3}, E_{1}\right], E_{0}\right]\right]\right]\right]\right] }
\end{aligned}
$$$$
\text { (by using } x(1)_{03}^{i j}=0 \text { ) }
$$$$
\left.=2\left[\left[\left[\left[E_{3}, E_{1}\right], E_{2}\right],\left[\left[E_{3}, E_{1}\right], E_{0}\right]\right], E_{1}\right]+\left[E_{1},\left[\left[\left[E_{2}, E_{3}\right], E_{1}\right],\left[\left[E_{3}, E_{1}\right], E_{0}\right]\right]\right]\right]
$$$$
\text { (by } x(5)_{310}^{i j k}=0 \text {, and since, by Lemma 2.2.1 (iii), }
$$ 


$$
\left[\left[\left[E_{3}, E_{1}\right], E_{2}\right],\left[E_{3}, E_{1}\right]\right] \sim\left[\left[\left[E_{1}, E_{2}\right], E_{3}\right],\left[E_{3}, E_{1}\right]\right]
$$

$\left.\sim\left[\left[\left[\left[E_{1}, E_{2}\right], E_{3}\right], E_{1}\right], E_{3}\right] \sim\left[\left[\left[\left[E_{2}, E_{3}\right], E_{1}\right], E_{1}\right], E_{3}\right]=0\right)$

$=0$ (by $x(7)_{312}^{i j k}=0$ (see also Lemma 2.2.1 (iii))),

${ }^{\prime} L_{2}^{M b}\left(x(5)_{210}^{i j k}\right)$

$\sim \quad\left[\left[E_{1},\left[E_{1}, E_{0}\right]\right],\left[E_{2}, E_{1}\right]\right] \sim\left[E_{1},\left[E_{1},\left[\left[E_{2}, E_{1}\right], E_{0}\right]\right]\right] \sim\left[E_{2}, x(3)_{1110}^{i i i j}\right]=0$ (by $\left.x(3)_{112}^{i i j}=0\right)$,

${ }^{\prime} L_{2}^{M b}\left(x(5)_{310}^{i j k}\right)$

$$
\begin{aligned}
\sim & {\left[\left[\left[\left[E_{2}, E_{3}\right],\left[E_{2}, E_{1}\right]\right],\left[\left[\left[E_{2}, E_{3}\right],\left[E_{2}, E_{1}\right]\right], E_{0}\right]\right],\left[E_{2}, E_{1}\right]\right] } \\
= & {\left[E_{2},\left(\left[\left[\left[\left[E_{2}, E_{3}\right],\left[E_{2}, E_{1}\right]\right],\left[\left[E_{2}, E_{3}\right],\left[\left[E_{2}, E_{1}\right], E_{0}\right]\right]\right], E_{1}\right]\right.\right.} \\
& \left.\left.-2\left[\left[E_{2}, E_{1}\right],\left[\left[E_{3},\left[E_{2}, E_{1}\right]\right],\left[\left[E_{2}, E_{3}\right],\left[\left[E_{2}, E_{1}\right], E_{0}\right]\right]\right]\right]\right)\right] \\
= & {\left[E_{2}, x(9)_{0123}^{i k k l}\right] \quad\left(\text { by Lemma 2.2.1 (iii) and } x(1)_{02}^{i j}=0\right) } \\
= & 0,
\end{aligned}
$$

$$
\begin{gathered}
{ }^{\prime} L_{2}^{M b}\left(x(7)_{123}^{i j k}\right) \sim^{\prime} L_{2}^{M b}\left(x(7)_{213}^{i j k}\right) \quad(\text { by Lemma 2.2.1 (iii)) } \\
\sim\left[E_{1}\left[E_{2}, E_{3}\right]\right]-\left[E_{3}\left[E_{2}, E_{1}\right]\right]=0 \quad\left(x(1)_{13}^{i j}=0\right),
\end{gathered}
$$

${ }^{\prime} L_{1}^{M b}\left(x(5)_{210}^{i j k}\right) \sim\left[\left[E_{2},\left[E_{2},\left[E_{1}, E_{0}\right]\right]\right], F_{1}\right] \sim\left[E_{2},\left[E_{2}, E_{0}\right]\right]=0$ (by Lemma 2.2.1 (iii)) and

$$
\begin{aligned}
& { }^{\prime \prime} L_{1}^{M b}\left(x(3)_{220}^{i j j}\right) \\
& \sim \quad\left[\left[E_{2}, E_{1}\right],\left[\left[E_{2}, E_{1}\right],\left[E_{0}, E_{1}\right]\right]\right] \\
& \sim \quad\left[\left[\left[E_{2}, E_{1}\right],\left[\left[E_{2}, E_{1}\right], E_{0}\right]\right], E_{1}\right] \quad \text { (by Lemma 2.2.1 (iii)) } \\
& =x(5)_{210}^{i j k}=0 .
\end{aligned}
$$

Thus we get (4.1.2) in the present case. In other cases, the corresponding calculation can be carried out similarly and, usually, much more easily.

Q. E. D.

4.5. As an immediate consequence of Theorem 3.5.1 and Theorem 4.1.1, we have

Theorem 4.5.1. Let $(\mathscr{E}, \Pi, p)$ be of affine ABCD type. Then the Lie superalgebra $\mathscr{G}(\mathscr{E}, \Pi, p)$ can also be defined by generators $H \in \mathscr{H}, E_{i}, F_{i}(0 \leq i \leq n)$, parities $p(\boldsymbol{H})=0, p\left(\boldsymbol{E}_{i}\right)=p\left(\boldsymbol{E}_{i}\right)=p\left(\alpha_{i}\right)$ and relations: 


$$
\text { (S1), (S2), (S3), (S4) (a) }(1 \leq a \leq 11),(\text { S5) (a) }(1 \leq a \leq 11)
$$

and

$$
\begin{aligned}
& x(k ; \delta)=0 \\
& y(k ; \delta)=0
\end{aligned}
$$$$
\text { if } k>0 \text { and } \operatorname{dim}\left(\operatorname{ker} \Psi\left[\mathscr{G}^{\natural}, \mathscr{G}\right] \cap \mathscr{G}_{k \delta}^{\natural}\right) \neq 0 \text {, }
$$$$
\text { if } k>0 \text { and } \operatorname{dim}\left(\operatorname{ker} \Psi\left[\mathscr{G}^{\natural}, \mathscr{G}\right] \cap \mathscr{G}_{-k \delta}^{\natural}\right) \neq 0
$$

where $x(k ; \delta)$ (resp. $y(k ; \delta))$ is an element of $\tilde{\mathscr{N}}^{+} \cap \tilde{\mathscr{G}}_{k \delta}$ (resp. $\left.\tilde{\mathscr{N}}^{-} \cap \tilde{\mathscr{G}}_{-k \delta}\right)$ such that $\Psi\left[\tilde{\mathscr{G}}, \mathscr{G}^{\natural}\right](x(k ; \delta))$ (resp. $\left.\Psi\left[\tilde{\mathscr{G}}, \mathscr{G}^{\natural}\right](y(k ; \delta))\right)$ spans the one dimensional $\operatorname{ker} \Psi\left[\mathscr{G}^{\natural}, \mathscr{G}\right] \cap \mathscr{G}_{k \delta}^{\natural}$ (resp. $\left.\operatorname{ker} \Psi\left[\mathscr{G}^{\natural}, \mathscr{G}\right] \cap \mathscr{G}_{-k \delta}^{\natural}\right)$.

\section{§5. Relations for $\mathrm{D}(2,1 ; x)^{(1)}, \mathrm{F}(4)^{(1)}$ and $\mathrm{G}(3)^{(1)}$}

5.1. In $\S 5$, we shall prove the counterpart of Theorem 4.5 .1 for $\mathscr{G}(\mathscr{E}, \Pi, p)$ of type $\mathrm{D}(2,1 ; x)^{(1)}, \mathrm{F}(4)^{(1)}$ and $\mathrm{G}(3)^{(1)}$ (see also 1.6 ).

We first deal with $\mathscr{G}(\mathscr{E}, \Pi, p)$ 's isomorphic to $\mathrm{D}(2 ; 1, x)^{(1)},(x \neq 0,-1)$. The argument needed in obtaining their defining relations is almost the same as in the case of affine ABCD-type. We first introduce tools which are the counterparts of the ones used to prove Theorem 4.5.1.

(a) We first define an auxiliary datum $\left(\mathscr{E}^{\dagger}, \Pi^{\dagger}=\left\{\alpha_{0}^{\dagger}, \alpha_{1}^{\dagger}, \alpha_{2}^{\dagger}, \alpha_{3}^{\dagger}\right\}\right)$. Let $\mathscr{E}^{\dagger}$ be a C-linear space with a basis $\left\{\alpha_{0}^{\dagger}, \alpha_{1}^{\dagger}, \alpha_{2}^{\dagger}, \alpha_{3}^{\dagger}, \Lambda_{0}^{\dagger}\right\}$ and a symmetric form (, ) such that the corresponding Dynkin diagram is

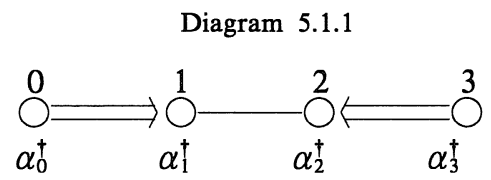

and that $\left(\Lambda_{0}^{\dagger}, \alpha_{j}^{\dagger}\right)=\delta_{0 j},\left(\Lambda_{0}^{\dagger}, \Lambda_{0}^{\dagger}\right)=0$.

(b) Let Data $\left(\mathrm{D}(2,1 ; x)^{(1)}\right)$ be a set of six data whose Dynkin diagrams are given in Figure 5.1.1 (see below). For $(\mathscr{E}, \Pi, p) \in \operatorname{Data}\left(\mathrm{D}(2,1 ; \boldsymbol{x})^{(1)}\right)$, we always assume $\operatorname{dim} \mathscr{E}=5$. For each datum $\left(\mathscr{E}, \Pi=\left\{\alpha_{0}, \alpha_{1}, \alpha_{2}, \alpha_{3}\right\}, p\right) \in \operatorname{Data}(\mathbb{D}(2,1$; $\left.x)^{(1)}\right)$, the numbering of elements of $\Pi$ is given by letting the $i$-th simple root $\alpha_{i}$ correspond to the vertex labeled as $i+j$ or $i$ in the corresponding Dynkin diagram. The C-linear space $\mathscr{E}$ is spanned by a basis $\Pi \cup\left\{\Lambda_{0}\right\}$ where $\Lambda_{0}$ is an element such that $\left(\alpha_{j}, \Lambda_{0}\right)=\delta_{0 j}$ and $\left(\Lambda_{0}, \Lambda_{0}\right)=0$. We also define a linear map $\iota: \mathscr{E} \rightarrow \mathscr{E}^{\dagger}$ as follows. If $\alpha_{i}$ corresponds to the vertex labeled $i+j$ (resp. $i$ ), then we put $\iota\left(\alpha_{i}\right)=$ $\alpha_{i}^{\dagger}+\alpha_{j}^{\dagger}$ (resp. $\iota\left(\alpha_{i}\right)=\alpha_{i}^{\dagger}$ ). We also put $\iota\left(\Lambda_{0}\right)=\Lambda_{0}^{\dagger}$, 
(c) Let $(\mathscr{E}, \Pi, p) \in \operatorname{Data}\left(\mathrm{D}(2,1 ; x)^{(1)}\right)$ and $i=0,1,2,3$. If there is an arrow labeled as $s_{i}$ between $(\mathscr{E}, \Pi, p)$ and a datum $\left(\mathscr{E}^{\prime}, \Pi^{\prime}, p^{\prime}\right)$, let $\left({ }^{s_{i}} \mathscr{E},{ }^{s_{i}} \Pi,{ }^{s_{i}} p\right)$ be $\left(\mathscr{E}^{\prime}, \Pi^{\prime}, p^{\prime}\right)$. Otherwise, let $\left({ }^{s_{i}} \mathscr{E},{ }^{s_{i}} \Pi,{ }^{s_{i}} p\right)=(\mathscr{E}, \Pi, p)$.

(d) Let ${ }^{s_{i}} P$ be the root lattice of $\left({ }^{s_{i}} \mathscr{E},{ }^{s_{i}} \Pi,{ }^{s_{i}} p\right)$. Define a parity preserving lattice isometry $f_{i}: P \rightarrow{ }^{s_{i}} P$ by

$$
f_{i}(\alpha)=\iota^{-1}\left(\iota(\alpha)-\frac{2\left(\iota(\alpha), \alpha_{i}^{\dagger}\right)}{\left(\alpha_{i}^{\dagger}, \alpha_{i}^{\dagger}\right)} \alpha_{i}^{\dagger}\right) \quad(\alpha \in P)
$$

(see Definition 2.2.4). Then $f_{i}$ is uniquely extended to a parity preserving isometry $s_{i}: \mathscr{E} \rightarrow^{s_{i}} \mathscr{E}$ (see Lemma 2.2.5).

Figure 5.1.1
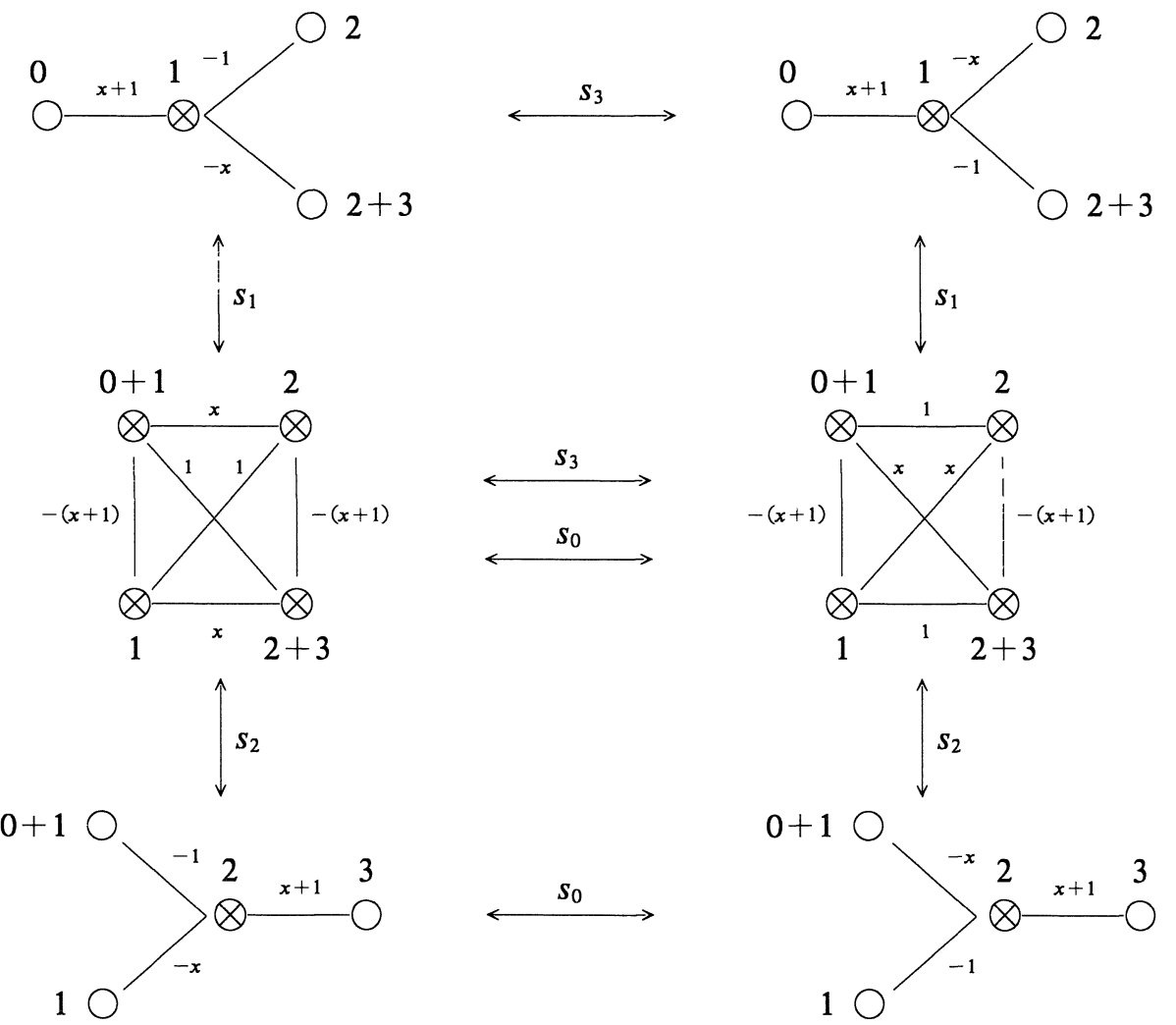

Then we have: 
Theorem 5.1.1. Let $(\mathscr{E}, \Pi, p) \in \operatorname{Data}\left(\mathrm{D}(2,1 ; \boldsymbol{x})^{(1)}\right)$. Then $\mathscr{G}(\mathscr{E}, \Pi, p)$ can also be defined by generators $H \in \mathscr{H}, E_{i}, F_{i}(0 \leq i \leq 3)$, parities $p(H)=0, p\left(E_{i}\right)=$ $p\left(F_{i}\right)=p\left(\alpha_{i}\right)$ and relations:

$$
\text { (S1), (S2), (S3), (S4) (a), (S5) (a) }(a=1,2,3,7) \text {, }
$$

$(\mathrm{S} 4)(8 x) \quad\left[\left[\left[E_{j}, E_{i}\right],\left[E_{j}, E_{k}\right]\right],\left[E_{j}, E_{l}\right]\right]=x\left[\left[\left[E_{j}, E_{i}\right],\left[E_{j}, E_{l}\right]\right],\left[E_{j}, E_{k}\right]\right]$

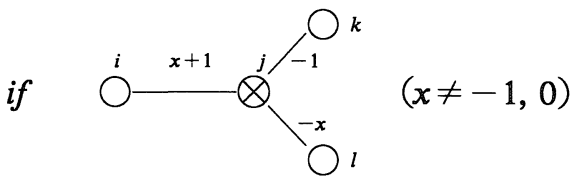

and

(S5) (8x) The same relation with $F_{r}$ 's in place of $E_{r}$ 's of (S4) (8x).

Proof. This theorem follows by exactly the same argument used in the proof of Theorem 4.5.1. We note that, by exactly the same argument as in the proof of Proposition 3.4.1, it is verified that $\mathscr{G}(\mathscr{E}, \Pi, p)$ is the maximal affine admissible Lie superalgebra. Here we only give examples of direct calculation needed in the proof.

Keep the notation in Step 2 of the proof of Theorem 4.1.1. Let $(\mathscr{E}, \Pi, p) \in$ $\operatorname{Data}\left(\mathrm{D}(2,1 ; \boldsymbol{x})^{(1)}\right)$ be the datum whose Dynkin diagram is the first one on the left of Figure 5.1.1. Then the Dynkin diagram of $\left({ }^{s_{1}} \mathscr{E},{ }^{s_{1}} \Pi,{ }^{s_{1}} p\right)$ is the second one on the left of Figure 5.1.1. We abbreviate $L_{1}^{M b}(\mathscr{E}, \Pi, p)$ and $L_{1}^{M b}\left({ }^{s_{1}} \mathscr{E},{ }^{s_{1}} \Pi,{ }^{s_{1}} p\right)$ to $L_{1}^{M b}$ and ' $L{ }_{1}^{M b}$ respectively.

We have:

$$
\begin{aligned}
& L_{1}^{M b}\left(x(8 x)_{0123}^{i j k l}\right) \\
& \quad \sim \quad\left[\left[E_{0}, E_{2}\right], E_{3}\right]-x\left[\left[E_{0}, E_{3}\right], E_{2}\right]=x(7)_{123}^{i j k}=0 .
\end{aligned}
$$

We also have:

$$
{ }^{\prime} L_{1}^{M b}\left(x(7)_{023}^{i j k}\right) \sim x(8 x)_{0123}^{i j k l}=0 .
$$

Thus we get (4.1.2) in the case of $\operatorname{Data}\left(\mathrm{D}(2,1 ; x)^{(1)}\right)$.

Q.E. D.

5.2. Here we deal with $F(4)^{(1)}=L(F(4), 1)$. We use the same argument as in 5.1 with Diagram 5.2.1 and Figure 5.2.1 in place of Diagram 5.1.1 and Figure 5.1.1. 
Diagram 5.2.1

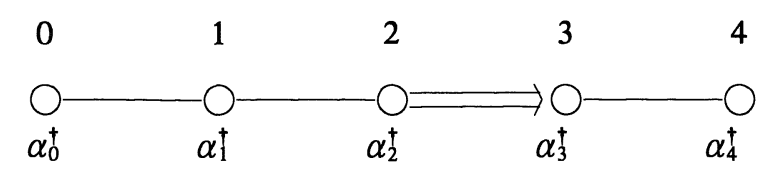

Let Data $\left(\mathrm{F}(4)^{(1)}\right)$ be the set of twelve data such that their Dynkin diagrams are given in Figure 5.2.1 (below). For $(\mathscr{E}, \Pi, p) \in \operatorname{Data}\left(\mathrm{F}(4)^{(1)}\right)$, we always assume that $\operatorname{dim} \mathscr{E}=6$ and $(\alpha, \alpha)=4$ for the simple root $\alpha$ corresponding to the leftmost vertex in the diagram. The numbering of simple roots is given as in (b) of 5.1. For example, the first one on the rightmost side satisfies $\iota\left(\alpha_{0}\right)=\alpha_{0}^{\dagger}+\alpha_{1}^{\dagger}+\alpha_{2}^{\dagger}, \iota\left(\alpha_{j}\right)=\alpha_{j}^{\dagger}$ $(j=1,3,4), \iota\left(\alpha_{2}\right)=\alpha_{2}^{\dagger}+\alpha_{3}^{\dagger}$.

Figure 5.2.1
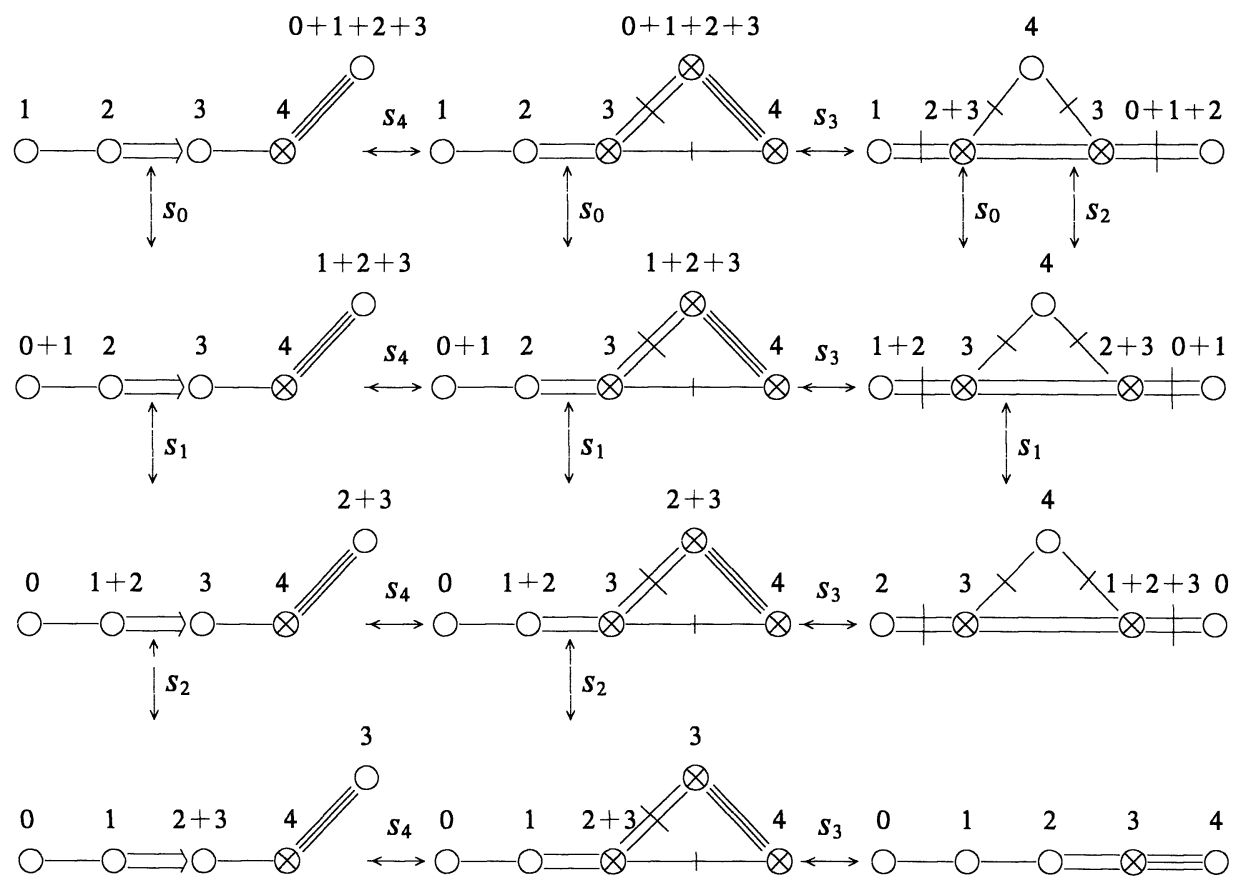

Theorem 5.2.1. Let $(\mathscr{E}, \Pi, p) \in \operatorname{Data}\left(\mathrm{F}(4)^{(1)}\right)$. Then $\mathscr{G}(\mathscr{E}, \Pi, p)$ can also be defined by generators $H \in \mathscr{H}, E_{i}, F_{i}(0 \leq i \leq 4)$, parities $p(H)=0, p\left(E_{i}\right)=p\left(F_{i}\right)=$ $p\left(\alpha_{i}\right)$ and relations: 
(S1), (S2), (S3), (S4) (a), (S5) (a) $(a=1,2,3,4,5,7)$,

$(\mathrm{S} 4)(12) \quad\left[\left[\left[\left[\left[\left[\left[\left[\left[\left[E_{i}, E_{j}\right], E_{k}\right], E_{l}\right], E_{k}\right], E_{j}\right], E_{k}\right], E_{l}\right], E_{k}\right], E_{j}\right], E_{k}\right]=0$

if

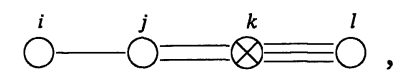

$(\mathrm{S} 4)(13) \quad\left[\left[\left[\left[\left[E_{l}, E_{k}\right], E_{j}\right], E_{i}\right], E_{k}\right], E_{j}\right]=2\left[\left[\left[\left[\left[E_{l}, E_{k}\right], E_{j}\right], E_{i}\right], E_{j}\right], E_{k}\right]$

if

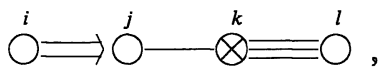

and

(S5) (b) $(b=12,13) \quad$ The same relation with $F_{r}$ 's in place of $E_{r}$ 's of $(\mathbf{S} 4)(b)$.

Proof. This theorem follows by exactly the same argument used in the proof of Theorem 4.5.1 and Theorem 5.1.1. We note that, since $(\rho, \delta) \neq 0, \mathscr{G}(\mathscr{E}, \Pi, p)$ itself is the maximal affine-admissible algebra. Here we only give examples of direct calculation needed in the proof.

Let $(\mathscr{E}, \Pi, p) \in \operatorname{Data}\left(\mathrm{F}(4)^{(1)}\right)$ be the datum whose Dynkin diagram is the first one on the leftmost of Figure 5.2.1. Then, letting $w_{1}=s_{3} s_{2} s_{1} s_{3} s_{2} s_{3} s_{4}$, the Dynkin diagram of $\left({ }^{w} \mathscr{E},{ }^{w} \Pi,{ }^{w_{1}} p\right)$ is the fourth one on the rightmost side of Figure 5.2.1.

Suppose $L_{4}^{M b}=L_{4}^{M b}(\mathscr{E}, \Pi, p)$. We note that $s_{4}\left(\alpha_{0}\right)=\alpha_{0}+\alpha_{4}, s_{4}\left(\alpha_{1}\right)=\alpha_{1}, s_{4}\left(\alpha_{2}\right)$ $=\alpha_{2}, s_{4}\left(\alpha_{3}\right)=\alpha_{3}+\alpha_{4}$ and $s_{4}\left(\alpha_{4}\right)=-\alpha_{4}$. Then we have

$$
\begin{aligned}
\left.L_{4}^{M b}(x(13))_{2340}^{i j k l}\right) & \\
= & L_{4}^{M b}\left(\left[\left[\left[\left[\left[E_{0}, E_{4}\right], E_{3}\right], E_{2}\right], E_{4}\right], E_{3}\right]-2\left[\left[\left[\left[\left[E_{0}, E_{4}\right], E_{3}\right], E_{2}\right], E_{3}\right], E_{4}\right]\right) \\
\sim & \quad\left[\left[\left[\left[E_{0},\left[E_{3}, E_{4}\right]\right], E_{2}\right], F_{4}\right],\left[E_{3}, E_{4}\right]\right]-2\left[\left[\left[\left[E_{0},\left[E_{3}, E_{4}\right]\right], E_{2}\right],\right.\right. \\
& {\left.\left.\left[E_{3}, E_{4}\right]\right], F_{4}\right] } \\
& \quad\left(\text { since }\left[\left[E_{0}, E_{4}\right], F_{4}\right] \sim E_{0}\right) \\
\sim & -\left[\left[\left[E_{0}, E_{3}\right], E_{2}\right],\left[E_{3}, E_{4}\right]\right]-2\left[\left[\left[E_{0},\left[E_{3}, E_{4}\right]\right], E_{2}\right], E_{3}\right] \\
\sim & {\left[\left[\left[E_{0}, E_{3}\right], E_{2}\right],\left[E_{3}, E_{4}\right]\right]+\left[\left[\left[E_{4},\left[E_{3}, E_{0}\right]\right], E_{2}\right], E_{3}\right] \quad\left(\text { by } x(7)_{340}^{i j k}=0\right) } \\
= & {\left[E_{3},\left[\left[\left[E_{0}, E_{3}\right], E_{2}\right], E_{4}\right]\right]+\left[\left[\left[E_{4},\left[E_{3}, E_{0}\right]\right], E_{2}\right], E_{3}\right] \quad\left(\text { by } x(4)_{032}^{i j k}=0\right) } \\
= & 0 .
\end{aligned}
$$

Put ${ }^{\prime} L_{4}^{M b}=L_{4}^{M b}\left({ }^{s_{4}} \mathscr{E},{ }^{s_{4}} \Pi,{ }^{s_{4}} p\right)$. Then we have

$$
\begin{aligned}
& { }^{\prime} L_{4}^{M b}\left(x(4)_{230}^{i j k}\right)={ }^{\prime} L_{4}^{M b}\left(\left[\left[\left[E_{2}, E_{3}\right], E_{0}\right], E_{3}\right]\right) \\
& \quad=\left[\left[\left[E_{2},\left[E_{3}, E_{4}\right]\right],\left[E_{0}, E_{4}\right]\right],\left[E_{3}, E_{4}\right]\right] \\
& \quad \sim\left[\left[\left[\left[\left[E_{0}, E_{4}\right], E_{3}\right], E_{4}\right], E_{2}\right],\left[E_{3}, E_{4}\right]\right] \\
& \quad=\left[\left[\left[\left[\left[\left[E_{0}, E_{4}\right], E_{3}\right], E_{4}\right], E_{2}\right], E_{3}\right], E_{4}\right] \\
& \quad=\left[\left(\left[\left[\left[\left[\left[E_{0}, E_{4}\right], E_{3}\right], E_{4}\right], E_{2}\right], E_{3}\right]-2\left[\left[\left[\left[\left[E_{0}, E_{4}\right], E_{3}\right], E_{4}\right], E_{3}\right], E_{4}\right]\right), E_{4}\right] \\
& \quad=\left[x(13)_{2340}^{i j k l}, E_{4}\right]=0
\end{aligned}
$$


where we note that, in $\mathscr{E}, \alpha_{0}=\iota^{-1}\left(\alpha_{0}^{\dagger}+\alpha_{1}^{\dagger}+\alpha_{2}^{\dagger}+\alpha_{3}^{\dagger}\right)$.

Suppose $L_{3}^{M b}=L_{3}^{M b}\left({ }^{w_{1}} \mathscr{E},{ }^{w_{1}} \Pi,{ }^{w_{1}} p\right)$. Then we have

$$
\begin{gathered}
L_{3}^{M b}\left(x(12)_{1234}^{i j k l}\right)=L_{3}^{M b}\left(\left[\left[\left[\left[\left[\left[\left[\left[\left[\left[E_{1}, E_{2}\right], E_{3}\right], E_{4}\right], E_{3}\right], E_{2}\right], E_{3}\right], E_{4}\right], E_{3}\right], E_{2}\right], E_{3}\right]\right) \\
\sim \quad\left[\left[\left[\left[\left[\left[\left[\left[\left[E_{1},\left[E_{2}, E_{3}\right]\right], F_{3}\right],\left[E_{3}, E_{4}\right]\right], F_{3}\right],\left[E_{2}, E_{3}\right]\right], F_{3}\right],\left[E_{3}, E_{4}\right]\right],\right.\right. \\
\left.\left.\left.\quad F_{3}\right],\left[E_{2}, E_{3}\right]\right], F_{3}\right] \\
\sim \quad\left[\left[\left[\left[\left[E_{1}, E_{2}\right], E_{4}\right], E_{2}\right], E_{4}\right], E_{2}\right] \\
=\quad\left[\left[\left[\left[E_{1}, E_{2}\right], E_{4}\right],\left[E_{2}, E_{4}\right]\right], E_{2}\right] \sim x(5)_{421}^{i j k}=0 .
\end{gathered}
$$

Put ${ }^{\prime} L_{3}^{M b}=L_{3}^{M b}\left({ }^{s_{3} w_{1}} \mathscr{E},{ }^{s_{3} w_{1}} \Pi,{ }^{s_{3} w_{1}} p\right)$. Then we have

$$
\begin{aligned}
{ }^{\prime} L_{3}^{M b} & \left(x(5)_{421}^{i j k}\right)={ }^{\prime} L_{3}^{M b}\left(\left[\left[\left[\left[E_{1}, E_{2}\right], E_{4}\right],\left[E_{2}, E_{4}\right]\right], E_{2}\right]\right) \\
& \sim\left[\left[\left[\left[E_{1},\left[E_{2}, E_{3}\right]\right],\left[E_{4}, E_{3}\right]\right],\left[\left[E_{2}, E_{3}\right],\left[E_{4}, E_{3}\right]\right]\right],\left[E_{2}, E_{3}\right]\right] \\
& \sim\left[\left[\left[\left[\left[\left[E_{1},\left[E_{2}, E_{3}\right]\right],\left[E_{4}, E_{3}\right]\right],\left[E_{2}, E_{3}\right]\right],\left[E_{4}, E_{3}\right]\right],\left[E_{2}, E_{3}\right]\right]\right. \\
\quad= & {\left[\left[\left[\left[\left[\left[\left[\left[\left[\left[E_{1}, E_{2}\right], E_{3}\right], E_{4}\right], E_{3}\right], E_{2}\right], E_{3}\right], E_{4}\right], E_{3}\right], E_{2}\right], E_{3}\right] } \\
\quad= & x(12)_{1234}^{i j k l}=0 .
\end{aligned}
$$

Thus we get (4.1.2) in the case of $\operatorname{Data}\left(\mathrm{F}(4)^{(1)}\right)$.

Q.E. D.

5.3. Here we treat the case of type $\mathrm{G}(3)^{(1)}$. Let Data $\left(\mathrm{G}(3)^{(1)}\right)$ be a set of five data whose Dynkin diagrams are given below:

\section{Figure 5.3.1}

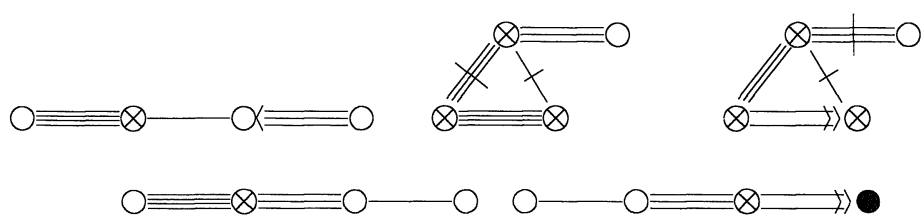

For $(\mathscr{E}, \Pi, p) \in \mathrm{G}(3)^{(1)}$, we always assume $\operatorname{dim} \mathscr{E}=5$.

Theorem 5.3.1. Let $(\mathscr{E}, \Pi, p) \in \operatorname{Data}\left(\mathrm{G}(3)^{(1)}\right)$. Then $\mathscr{G}(\mathscr{E}, \Pi, p)$ can be also defined by generators $H \in \mathscr{H}, E_{i}, F_{i}(0 \leq i \leq 3)$, parities $p(H)=0, p\left(E_{i}\right)=p\left(F_{i}\right)=$ $p\left(\alpha_{i}\right)$ and relations:

$$
\text { (S1), (S2), (S3), (S4)(a), (S5) (a) }(a=1,2,3,4,7)
$$

and

$(\mathrm{S} 4)(14) \quad\left[\left[\left[E_{i}, E_{j}\right],\left[\left[E_{i}, E_{j}\right],\left[\left[E_{i}, E_{j}\right], E_{k}\right]\right]\right], E_{j}\right]=0$ 


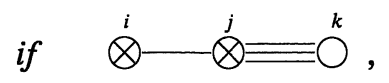

(S4) (15) $\left[E_{j},\left[E_{k},\left[E_{k},\left[E_{j}, E_{i}\right]\right]\right]\right]=\left[E_{k},\left[E_{j},\left[E_{k},\left[E_{j}, E_{i}\right]\right]\right]\right]$

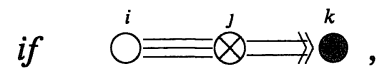

$(\mathrm{S} 4)(16) 2\left[\left[\left[\left[\left[E_{l}, E_{k}\right], E_{j}\right], E_{i}\right], E_{k}\right], E_{j}\right]=3\left[\left[\left[\left[\left[E_{l}, E_{k}\right], E_{j}\right], E_{i}\right], E_{j}\right], E_{k}\right]$

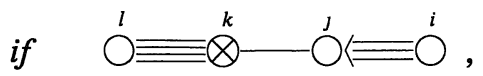

(S4) (17)

$\left[\left[\left[\left[\left[\left[\left[\left[\left[\left[\left[\left[\left[\left[\left[E_{i}, E_{j}\right], E_{k}\right], E_{l}\right], E_{k}\right], E_{j}\right], E_{k}\right], E_{l}\right], E_{k}\right], E_{j}\right], E_{k}\right], E_{l}\right], E_{k}\right], E_{j}\right], E_{k}\right]=0\right.$

if

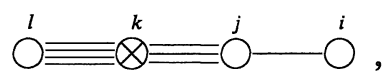

(S5) (b) $(14 \leq b \leq 17) \quad$ The same relation with $F_{r}$ 's in place of $E_{r}$ 's of $(\mathrm{S} 4)(b)$.

Proof. Let $\left(\mathscr{E}_{1}, \Pi_{1}, p_{1}\right)=\left(\mathscr{E}_{1}, \Pi_{1}=\left\{\alpha_{0}, \alpha_{1}, \alpha_{2}, \alpha_{3}\right\}, p_{1}\right)$ be the datum whose Dynkin diagram is the first one of the first row of Figure 5.3.1. We assume $\left(\alpha_{0}, \alpha_{0}\right)$ $=-8,\left(\alpha_{1}, \alpha_{1}\right)=0,\left(\alpha_{2}, \alpha_{2}\right)=2$ and $\left(\alpha_{3}, \alpha_{3}\right)=6$. Let $\delta=\alpha_{0}+2 \alpha_{1}+4 \alpha_{2}+2 \alpha_{3}$. Let $\Phi_{+}=\Phi\left(\mathscr{E}_{1}, \Pi_{1}, p_{1}\right) \cap P_{+}$be the set of positive roots of $\mathscr{G}=\mathscr{G}\left(\mathscr{E}_{1}, \Pi_{1}, p_{1}\right)$. Let $\left(\mathscr{E}_{0}\right.$, $\left.\Pi_{0}, p_{0}\right)$ be the datum such that $\Pi_{0}=\left\{\alpha_{1}, \alpha_{2}, \alpha_{3}\right\}, \operatorname{dim} \mathscr{E}_{0}=3$ and $p_{0}=p_{1}$. Then $\mathscr{G}\left(\mathscr{E}_{0}, \Pi_{0}, p_{0}\right)$ is the finite dimensional simple Lie superalgebra of type $G(3)$ (see $[\mathrm{K} 2])$. Let $\Phi_{0,+}$ be the set of positive roots of $\mathscr{G}\left(\mathscr{E}_{0}, \Pi_{0}, p_{0}\right)$. Then we have $\Phi_{0,+}$ $=\left\{a \alpha_{1}+b \alpha_{2}+c \alpha_{3} \mid(a, b, c)=(1,0,0),(1,1,0),(1,1,1),(1,2,1),(1,3,1)\right.$, $(1,3,2),(1,4,2),(2,4,2),(0,0,1),(0,1,1),(0,3,2),(0,2,1),(0,3,1)$, $(0,1,0)\}$. Then we have $\Phi_{+}=\Phi_{0,+} \cup\left\{x \delta \pm \theta \mid x>0, \theta \in \Phi_{0,+}\right\} \cup\{y \delta \mid y>0\}$. For $i$ $=0,2,3$, we define $s_{i}: \mathscr{E}_{1} \rightarrow \mathscr{E}_{1}$ by $s_{i}(\gamma)=\gamma-\frac{2\left(\gamma, \alpha_{i}\right)}{\left(\alpha_{i}, \alpha_{i}\right)} \alpha_{i}$. Put

$$
\begin{aligned}
& \Phi_{+}^{b}=\Phi_{+} \cup \cup_{i=0}^{3}\left(\Phi_{+}+\alpha_{i}\right), \\
& \Phi_{+}^{\#}=\left\{\gamma \in \Phi_{+}^{b} \mid(\gamma, \gamma)=2(\rho, \gamma)\right\} .
\end{aligned}
$$

It is clear from Proposition 1.2.2 that, if $\mathscr{G}^{\sharp} \in I\left(\mathscr{E}_{1}, \Pi_{1}, p_{1}\right)$ satisfies $\operatorname{dim} \mathscr{G}_{\alpha}^{\#}=$ $\operatorname{dim} \mathscr{G}_{\alpha}$ for any $\alpha \in \Phi_{+}^{\#}$, then $\mathscr{G}^{\sharp}=\mathscr{G}$. Since $|2(\rho, \delta)|=12$, sufficiently large elements of $\Phi_{+}^{b}$ do not belong to $\Phi_{+}^{\#}$. By direct calculation, we have

$$
\begin{aligned}
\Phi_{+}^{\#}= & \left\{\alpha_{1}, 2 \alpha_{1}, \alpha_{0}, \alpha_{3}, \alpha_{2}, \alpha_{0}+3 \alpha_{1}+4 \alpha_{2}+\alpha_{3}, \alpha_{1}+2 \alpha_{2}, \alpha_{0}+\alpha_{1}+\alpha_{2}+\alpha_{3}, 2 \alpha_{0}+3 \alpha_{1}\right. \\
& \left.+5 \alpha_{2}+2 \alpha_{3}, \alpha_{0}+2 \alpha_{1}+4 \alpha_{2}+4 \alpha_{3}, \alpha_{2}+2 \alpha_{3}, \alpha_{0}+2 \alpha_{1}+2 \alpha_{2}+\alpha_{3}, 4 \alpha_{2}+\alpha_{3}\right\} .
\end{aligned}
$$

Let $r_{+}^{b}$ be the ideal of $\tilde{N}^{+}$generated by the relations $(\mathbf{S} 4)(b)(b=1,2,3,16)$. Let $r_{-}^{b}$ be the ideal of $\tilde{N}^{-}$generated by the relations $(\mathbf{S} 5)(b)(b=1,2,3,16)$. By 
using Lemma 2.2.2, we can see $r_{ \pm}^{b} \subset \operatorname{ker} \Psi\left[\mathscr{G}^{\#}, \mathscr{G}\right] \cap \tilde{N}^{ \pm}$. It is easily verified that $r^{b}=r_{-}^{b} \oplus r_{+}^{b}$ is an ideal of $\tilde{\mathscr{G}}$. Let $\mathscr{G}^{b}=\tilde{\mathscr{G}} / r^{b}$. Then $\mathscr{G}^{b} \in I\left(\mathscr{E}_{1}, \Pi_{1}, p_{1}\right)$. By Proposition 2.2.7, we may define the automorphisms $L_{i}^{b}: \mathscr{G}^{b} \rightarrow \mathscr{G}^{b}(i=0,2,3)$ by

$$
L_{i}^{b}=\exp \left(\operatorname{ad} E_{i}\right) \exp \left(\operatorname{ad}\left(-\frac{2}{\left(\alpha_{i}, \alpha_{i}\right)} F_{i}\right)\right) \exp \left(\operatorname{ad} E_{i}\right)
$$

We also have $L_{i}^{b}\left(\mathscr{G}_{\alpha}^{b}\right)=\mathscr{G}_{s_{i}(\alpha)}^{b}$ where $s_{i}(\alpha)=\alpha-\frac{2\left(\alpha, \alpha_{i}\right)}{\left(\alpha_{i}, \alpha_{i}\right)} \alpha_{i}$. By the same formula as in (5.3.1), we also have the automorphism $L_{i}: \mathscr{G} \rightarrow \mathscr{G}(i=0,2,3)$ such that $L_{i}\left(\mathscr{G}_{\alpha}\right)$ $=\mathscr{G}_{s_{i}(\alpha)}$. Note $\operatorname{dim} \mathscr{G}_{\alpha}^{b}=\operatorname{dim} \mathscr{G}_{\alpha}$ for $\alpha \in \cup_{i=0}^{3} \mathbf{Z}_{+} \alpha_{i}$. Let $\beta \in \Phi_{+}^{\#}$ be a minimal element with respect to the order $\leq\left(\right.$ see 1.2) among $\left\{\beta^{\prime} \in \Phi_{+}^{\#} \mid \operatorname{dim} \mathscr{G}_{\beta^{\prime}}^{b}>\operatorname{dim} \mathscr{G}_{\beta^{\prime}}\right\}$. Then $\beta$ satidfies:

$$
\beta \in \Phi_{+}^{b} \quad \text { and }\left(\beta, \alpha_{0}\right) \geq 0, \quad\left(\beta, \alpha_{2}\right) \leq 0, \quad\left(\beta, \alpha_{3}\right) \leq 0
$$

since $s_{i}(\beta) \geq \beta(i=0,2,3)$. The unique element of $\Phi_{+}^{\#}$ satisfying (5.3.2) is $\alpha_{0}+$ $2 \alpha_{1}+2 \alpha_{2}+\alpha_{3}$. However, using the relation (S4) (16), we have $\operatorname{dim} \mathscr{G}_{\alpha_{0}+2 \alpha_{1}+2 \alpha_{2}+\alpha_{3}}^{b}$ $\leq 1$. Hence $\operatorname{dim} \mathscr{G}_{\alpha_{0}+2 \alpha_{1}+2 \alpha_{2}+\alpha_{3}}^{b}=\operatorname{dim} \mathscr{G}_{\alpha_{0}+2 \alpha_{1}+2 \alpha_{2}+\alpha_{3}}(=1)$. Hence such $\beta$ doesn't exist. Hence $\mathscr{G}^{b}$ is isomorphic to $\mathscr{G}$.

For the other datum of Figure 5.3.1 than $\left(\mathscr{E}_{1}, \Pi_{1}, p_{1}\right)$, we can prove the statement by iterating the isomorphisms corresponding to the super-reflections (see Proposition 2.2.6).

Q. E. D.

\section{§6. q-Analogues of the Defining Relations}

6.1. Let $\mathbf{C}(q)$ be the rational function field in a variable $q$ over $\mathbf{C}$. The letter $\sigma$ denotes the generator of $\mathbf{Z}_{2}$. Assume $V$ to be a $\mathbf{Z}_{2}$-graded $\mathbf{C}(q)$ (resp. C)-algebra. We also view $V$ as a Lie $\mathbf{C}(q)$ (resp. C)-superalgebra by putting

$$
[X, Y]=X Y-(-1)^{p(X) p(Y)} Y X
$$

where $X$ and $Y$ are homogeneous elements of $V$ (see 1.1). Since $\mathbf{Z}_{2}$ acts on $V$ by $\sigma(X)=(-1)^{p(X)} X$ for a homogeneous element $X$, we can consider a $\mathbf{C}(q)$ (resp. C)algebra $V^{\sigma}=V \oplus \sigma V$ such that $\sigma X \sigma=(-1)^{p(X)} X$ for any homogeneous element $X \in$ $V$ (see [Y1]). We call $V^{\sigma}$ the extension of $V$ with $\sigma$. For a $\mathbf{Z}_{2}$-graded $\mathbf{C}(q)$ (resp. C)-algebra homomorphism $\varphi: V \rightarrow W$, define $\varphi^{\sigma}: V^{\sigma} \rightarrow W^{\sigma}$ by $\varphi^{\sigma}(X)=X(X \in V)$ and $\varphi^{\sigma}(\sigma)=\sigma$. Then $\varphi$ is an algebra homomorphism. We call $\varphi^{\sigma}$ the extension of $\varphi$ with $\sigma$.

Definition 6.1.1. Let $(\mathscr{E}, \Pi, p)$ be a datum. We say that a quadruple $(\mathscr{E}, \Pi$, $p, \Gamma)$ is a lattice datum if the following conditions are satisfied: 
(a) $\Gamma$ is a lattice in $\mathscr{E}$, i.e., $\Gamma$ is a $\mathbf{Z}$-span of a basis of $\mathscr{E}$.

(b) $\left(\gamma, \gamma^{\prime}\right) \in \mathbb{Z}$ for any $\gamma, \gamma^{\prime} \in \Gamma$.

(c) $\Pi \subset \Gamma$.

For a lattice datum $\left(\mathscr{E}, \Pi=\left\{\alpha_{0}, \ldots, \alpha_{n}\right\}, p, \Gamma\right)$, we define an associative $\mathbf{Z}_{2}$-graded $\mathbf{C}(q)$-algebra $U_{q}=\widetilde{U}_{q}(\mathscr{E}, \Pi, p, \Gamma)$ (with 1$)$ by generators:

$$
K_{r}(\gamma \in \Gamma), \quad E_{i}, F_{i}(0 \leq i \leq n),
$$

parities:

$$
p\left(K_{\gamma}\right)=0, \quad p\left(E_{i}\right)=p\left(\alpha_{i}\right), \quad p\left(F_{i}\right)=p\left(\alpha_{i}\right),
$$

and relations:

(QS1) $\quad K_{0}=1, K_{\gamma} K_{\gamma^{\prime}}=K_{\gamma+\gamma^{\prime}} \quad$ for all $\gamma, \gamma^{\prime} \in \Gamma$,

(QS2) $\quad K_{\gamma} E_{i} K_{\gamma}^{-1}=q^{\left(\gamma, \alpha_{i}\right)} E_{i}, K_{\gamma} F_{i} K_{\gamma}^{-1}=q^{-\left(\gamma, \alpha_{i}\right)} F_{i}$,

(QS3) $\quad\left[E_{i}, F_{j}\right]=\delta_{i j} \frac{K_{\alpha_{i}}-K_{\alpha_{i}}^{-1}}{q-q^{-1}}$.

Then the extension $\tilde{U}_{q}^{\sigma}=\tilde{U}_{q}^{\sigma}(\mathscr{E}, \Pi, p, \Gamma)$ of $\tilde{U}_{q}$ with $\sigma$ has a Hopf algebra structure $\left(\tilde{U}_{q}^{\sigma}, \Delta, S, \varepsilon\right)$ with comultiplication $\Delta$, antipode $S$, and counit $\varepsilon$ such that

$$
\begin{aligned}
& \Delta(\sigma)=\sigma \otimes \sigma, \Delta\left(K_{\gamma}\right)=K_{r} \otimes K_{\gamma}, \\
& \Delta\left(E_{i}\right)=E_{i} \otimes 1+K_{\alpha_{i}} \sigma^{p\left(\alpha_{i}\right)} \otimes E_{i}, \Delta\left(F_{i}\right)=F_{i} \otimes K_{\alpha_{i}}^{-1}+\sigma^{p\left(\alpha_{i}\right)} \otimes F_{i} \\
& S(\sigma)=\sigma, S\left(K_{\gamma}\right)=K_{r}^{-1}, S\left(E_{i}\right)=-K_{\alpha_{i}}^{-1} \sigma^{p\left(\alpha_{i}\right)} E_{i}, S\left(F_{i}\right)=-\sigma^{p\left(\alpha_{i}\right)} F_{i} K_{\alpha_{i}}, \\
& \varepsilon(\sigma)=1, \varepsilon\left(K_{\gamma}\right)=1, \varepsilon\left(E_{i}\right)=0, \varepsilon\left(F_{i}\right)=0 .
\end{aligned}
$$

Let $\tilde{U}_{q}^{+}$(resp. $\tilde{U}_{q}^{-}$) be a subalgebra (with 1 ) of $\tilde{U}_{q}$ generated by $E_{0}, \ldots, E_{n}$ (resp. $\left.F_{0}, \ldots, F_{n}\right)$. It is easily verified that $\widetilde{U}_{q}^{+}$(resp. $\widetilde{U}_{q}^{-}$) is a free algebra with generators $E_{0}, \ldots, E_{n}$ (resp. $F_{0}, \ldots, F_{n}$ ). Let $T$ be a subalgebra (with 1) of $\tilde{U}_{q}$ generated by $K_{r}(\gamma \in \Gamma)$. It can be verified that $K_{\gamma}(\gamma \in \Gamma)$ form a basis of $T$. Moreover we have a $\mathbf{C}(q)$-linear isomorphism:

$$
\widetilde{U}_{q}^{+} \otimes_{\mathbf{C}(q)}(T \oplus \sigma T) \otimes_{\mathbf{C}(q)} \tilde{U}_{q}^{-} \rightarrow \tilde{U}_{q}^{\sigma}
$$

$(X \otimes Z \otimes Y \rightarrow X Z Y$ ) (see $[Y 1])$. Denote the subalgebra $T \oplus \sigma T$ (resp. $\tilde{U}_{q}^{+} \otimes(T \oplus$ $\sigma T)$ ) of $\tilde{U}_{q}^{\sigma}$ by $T^{\sigma}$ (resp. $\left.\tilde{B}_{q}^{\sigma}\right)$. Let $\Gamma^{\vee}=\{\lambda \in \mathscr{E} \mid(\lambda, \gamma) \in \mathbb{Z}(\gamma \in \Gamma)\}$. Assume $M$ to be a left $T$-module. For $\gamma \in \Gamma^{\vee}$, let $M_{\gamma}=\left\{m \in M \mid K_{\mu} \cdot m=q^{(\gamma, \mu)} m\right.$ for any $\left.\mu \in \Gamma\right\}$. We note $\tilde{U}_{q}^{ \pm}=\oplus_{\alpha \in P_{+}}\left(\tilde{U}_{q}^{ \pm}\right)_{ \pm \alpha}$ where $T$ acts on $\tilde{U}_{q}^{+}$by $K_{\gamma}(X)=K_{\gamma} X K_{-\gamma}\left(X \in \tilde{U}_{q}^{ \pm}\right)$. 
6.2.

Proposition 6.2.1. (i) There is a unique symmetric form $\langle\rangle:, \widetilde{B}_{q}^{\sigma} \times \widetilde{B}_{q}^{\sigma} \rightarrow$ $\mathbf{C}(q)$ such that

(ia) $\left\langle X_{1} Z_{1}, X_{2} Z_{2}\right\rangle=\left\langle X_{1}, X_{2}\right\rangle\left\langle Z_{1}, Z_{2}\right\rangle$ for $X_{1}, X_{2} \in \tilde{U}_{q}^{+}$and $Z_{1}, Z_{2} \in T^{\sigma}$,

(ib) $\left\langle K_{\alpha} \sigma^{r_{1}}, K_{\beta} \sigma^{r_{2}}\right\rangle=q^{(\alpha, \beta)}(-1)^{r_{1} r_{2}}\left(\alpha, \beta \in \Gamma, r_{1}, r_{2} \in \mathbf{Z}\right)$,

(ic) $\left\langle E_{i}, E_{j}\right\rangle=\delta_{i j}$, and $\left\langle X_{1}, X_{2}\right\rangle=0$ if $X_{1} \in\left(\tilde{U}_{q}^{+}\right)_{\alpha}, X_{2} \in\left(\tilde{U}_{q}^{+}\right)_{\beta}$ and $\alpha \neq \beta$,

(id) $\left\langle\Delta\left(W_{1}\right), W_{2} \otimes W_{3}\right\rangle=\left\langle W_{1}, W_{2} W_{3}\right\rangle,\left\langle S\left(W_{1}\right), W_{2}\right\rangle=\left\langle W_{1}, S\left(W_{2}\right)\right\rangle$, $\left\langle 1, W_{2}\right\rangle=\varepsilon\left(W_{2}\right)$ for $W_{1}, W_{2}, W_{3} \in \widetilde{B}_{q}^{\sigma}$.

(ii) Let $\phi: \widetilde{U}_{q}^{-} \rightarrow \widetilde{U}_{q}^{+}$be the isomorphism defined by $\phi\left(F_{i}\right)=E_{i}$. Let $\theta: \tilde{U}_{q}^{+} \rightarrow$ $\widetilde{U}_{q}^{+}$be the anti-isomorphism defined by $\theta\left(E_{i}\right)=E_{i}$. For $\beta=\alpha_{i_{1}}+\cdots+\alpha_{i_{k}} \in P_{+}$, put $l(\beta)=k, r_{1}(\beta)=q^{\Sigma_{a<b}\left(\alpha_{i_{a}}, \alpha_{i_{b}}\right)}$ and $r_{2}(\beta)=(-1)^{\Sigma_{a<b} p\left(\alpha_{i_{a}}\right) p\left(\alpha_{i_{b}}\right)}$. Put $\Delta^{(2)}=(\Delta \otimes 1) \circ$ D. Let $X \in\left(\tilde{U}_{q}^{+}\right)_{\beta}$ and $Y \in\left(\tilde{U}_{q}^{-}\right)_{-\gamma}\left(\alpha, \gamma \in P_{+}\right)$. Write

$$
\begin{array}{r}
\Delta^{(2)}(X)=\sum X_{1} K_{\beta_{2}+\beta_{3}} \sigma^{p\left(\beta_{2}+\beta_{3}\right)} \otimes X_{2} K_{\beta_{3}} \sigma^{p\left(\beta_{3}\right)} \otimes X_{3} \\
\left(\beta=\beta_{1}+\beta_{2}+\beta_{3}, X_{i} \in\left(\tilde{U}_{q}^{+}\right)_{\beta_{i}}\right), \\
\begin{aligned}
\Delta^{(2)}(Y)=\sum Y_{1} \sigma^{p\left(\gamma_{2}+\gamma_{3}\right)} \otimes K_{-\gamma_{1}} \sigma^{p\left(\gamma_{3}\right)} Y_{2} \otimes Y_{3} K_{-\left(\gamma_{1}+\gamma_{2}\right)} \\
\left(\gamma=\gamma_{1}+\gamma_{2}+\gamma_{3}, Y_{i} \in\left(\tilde{U}_{q}^{-}\right)_{-\gamma_{i}}\right) .
\end{aligned}
\end{array}
$$

Then we have

$$
\begin{aligned}
& Y X=\sum\left(\frac{(-1)}{q-q^{-1}}\right)^{l\left(\gamma_{1}+\gamma_{3}\right)}(-1)^{l\left(\beta_{1}\right)} \frac{r_{2}\left(\beta_{1}\right)}{r_{1}\left(\beta_{1}\right)} q^{-\left(\beta_{1}, \beta_{2}+\beta_{3}\right)} \\
& (-1)^{p\left(\beta_{1}\right) p\left(\beta_{2}+\beta_{3}\right)} r_{2}\left(\gamma_{1}\right)(-1)^{p(\beta) p(\gamma)}\left\langle\theta\left(X_{1}\right), \phi\left(Y_{1}\right)\right\rangle \\
& r_{2}\left(\gamma_{3}\right)\left\langle X_{3}, \phi\left(Y_{3}\right)\right\rangle X_{2} K_{\beta_{3}-\gamma_{1}} Y_{2} .
\end{aligned}
$$

In [Y1], an essentially the same result as Proposition 6.2.1 is proved via the well-known argument due to Drinfeld $[\mathrm{D} 1]$. See also $[\mathrm{L} 1]$ and $[\mathrm{T}]$.

6.3. Let

$$
I^{+}=\left\{X \in \tilde{U}_{q}^{+} \mid\left\langle X, X_{1}\right\rangle=0 \quad \text { for any } X_{1} \in \tilde{U}_{q}^{+}\right\}
$$

It is clear that $I^{+}=\oplus_{\beta \in P_{+} \backslash(\Pi \cup\{0\})} I_{\beta}^{+}$where $I_{\beta}^{+}=I^{+} \cap\left(\tilde{U}_{q}^{+}\right)_{\beta}$. It is easily verified that $\operatorname{ker}\langle\rangle=,I^{+} T^{\sigma}$.

For $X \in\left(\widetilde{U}_{q}^{+}\right)_{\beta}$ with $\beta \in P_{+}$with $\Delta(X)=\sum_{\beta_{1}+\beta_{2}=\beta} X_{1} K_{\beta_{2}} \sigma^{p\left(\beta_{2}\right)} \otimes X_{2} \quad\left(\beta=\beta_{1}+\right.$ $\left.\beta_{2}, X_{i} \in\left(\tilde{U}_{q}^{+}\right)_{\beta_{i}}\right)$, we have 


$$
S(X)=(-1)^{l(\beta)} r_{1}(\beta) r_{2}(\beta) K_{-\beta} \sigma^{p(\beta)} \theta(X),
$$

and

$$
\Delta\left(\phi^{-1}(X)\right)=\sum_{\beta_{1}+\beta_{2}=\beta} \sigma^{p\left(\beta_{1}\right)} \phi^{-1}\left(X_{2}\right) \otimes \phi^{-1}\left(X_{1}\right) K_{-\beta_{2}} .
$$

By Proposition 6.2.1 and (6.3.1), we have:

Lemma 6.3.1. $\theta\left(I^{+}\right)=I^{+}$.

Put

$$
J^{+}=I^{+} T^{\sigma} \widetilde{U}_{q}^{-} \text {and } J^{-}=\tilde{U}_{q}^{+} T^{\sigma} I^{-}
$$

where $I^{-}=\phi^{-1}\left(I^{+}\right)$. Then we have:

\section{Lemma 6.3.2. $J^{+}$and $J^{-}$are Hopf ideals of $\tilde{U}_{q}^{\sigma}$.}

Proof. By using (6.2.1) and Lemma 6.3.1, and by (6.3.2), we see that $\tilde{U}_{q}^{-} I^{+}$ $\subset I^{+} T^{\sigma} \widetilde{U}_{q}^{-}$and $I^{-} \tilde{U}_{q}^{+} \subset \tilde{U}_{q}^{+} T^{\sigma} I^{-}$. Hence $J^{+}$and $J^{-}$are ideals. It is easily verified that $\Delta\left(J^{ \pm}\right) \subset J^{ \pm} \otimes \tilde{U}_{q}^{\sigma}+\tilde{U}_{q}^{\sigma} \otimes J^{ \pm}, S\left(J^{ \pm}\right)=J^{ \pm}$and $\varepsilon\left(J^{ \pm}\right)=\{0\} . \quad$ Q. E. D.

6.4. Define a Hopf $\mathbb{C}(q)$-algebra $U_{q}^{\sigma}=U_{q}^{\sigma}(\mathscr{E}, \Pi, p, \Gamma)=\left(U_{q}^{\sigma}, \Delta, S, \varepsilon\right)$ as the quotient Hopf algebra:

$$
U_{q}^{\sigma}=U_{q}^{\sigma}(\mathscr{E}, \Pi, p, \Gamma)=\tilde{U}_{q}^{\sigma} /\left(J^{+}+J^{-}\right)
$$

Let $\pi: \tilde{U}_{q}^{\sigma} \rightarrow U_{q}^{\sigma}$ be the canonical map. By abuse of notation, the elements $\pi(\sigma)$, $\pi\left(K_{\gamma}\right), \pi\left(E_{i}\right), \pi\left(F_{i}\right)$ (resp. the subalgebras $\pi(T)$ and $\pi\left(T^{\sigma}\right)$ ) will be denoted by $\sigma$, $K_{r}, E_{i}, F_{i}$ (resp. $T$ and $\left.T^{\sigma}\right)$. The subalgebras $\pi\left(\widetilde{U}_{q}^{+}\right)$and $\pi\left(\tilde{U}_{q}^{-}\right)$will be denoted by $U_{q}^{+}$and $U_{q}^{-}$. We note that

$$
\operatorname{ker} \pi_{\mid T^{\sigma}}=\{0\}, \operatorname{ker} \pi_{\mid \tilde{U}_{q}^{+}}=I^{+}, \operatorname{ker} \pi_{\mid \tilde{U}_{q}^{-}}=I^{-},
$$

and that there is a $\mathbb{C}(q)$-linear isomorphism:

$$
X_{q}^{+} \otimes_{\mathbf{C}(q)} T^{\sigma} \otimes_{\mathbf{C}(q)} U_{q}^{-} \rightarrow U_{q}^{\sigma}
$$

$(X \otimes Z \otimes Y \rightarrow X Z Y)$

6.5. For $\beta \in \boldsymbol{P}_{+}$, let $X_{\left(\beta, i_{\beta}\right)}$ (resp. $\left.Y_{\left(\beta, i_{\beta}\right)}\right) \quad\left(1 \leq i_{\beta} \leq \operatorname{dim}\left(\tilde{U}_{q}^{+}\right)_{\beta}\right)$ be a basis of $\left(\tilde{U}_{q}^{+}\right)_{\beta}$ (resp. $\left(\tilde{U}_{q}^{-}\right)_{-\beta}$ ). We assume $X_{(0,1)}$ and $Y_{(0,1)}$ to be 1. By (6.1.1), for $X \in \tilde{U}_{q}^{+}$and 
$Y \in \tilde{U}_{q}^{-}, Y X$ can be written uniquely as a sum

$$
\sum Q_{\left(\beta, i_{\beta}\right),(\alpha),\left(\gamma, j_{\gamma}\right)}(X, Y) X_{\left(\beta, i_{\beta}\right)} K_{\alpha} Y_{\left(\gamma, j_{\gamma}\right)} \in \widetilde{U}_{q}^{\sigma}
$$

where $\boldsymbol{Q}_{\left(\beta, i_{\beta}\right),(\alpha),\left(\gamma, j_{\gamma}\right)}(X, Y) \in \mathbf{C}(q)$. For $(X, Y) \in\left(\tilde{U}_{q}^{+}\right)_{\beta} \times\left(\tilde{U}_{q}^{-}\right)_{-\beta}$ with $\beta \in P_{+}$, put $Q_{\beta}(X, Y)=Q_{(0,1),(\beta),(0,1)}(X, Y) \in \mathbf{C}(q)$.

Proposition 6.5.1. Let $\left(\mathscr{E}, \Pi=\left\{\alpha_{0}, \ldots, \alpha_{n}\right\}, p, \Gamma\right)$ be a lattice datum. If $X \in$ $U_{q}^{+}$satisfies

$$
\left[X, F_{i}\right]=0 \text { for any } 0 \leq i \leq n,
$$

then $X=0$.

Proof. We may assume $X \in\left(U_{q}^{+}\right)_{\beta}$ for some $\beta \in P_{+}$. It is clear that $\left[X, F_{i_{1}} F_{i_{2}}\right.$ $\left.\cdots F_{i_{r}}\right]=0$ for all elements $\alpha_{i_{1}}, \alpha_{i_{2}}, \ldots, \alpha_{i_{r}} \in \boldsymbol{P}_{+}$. Hence

$$
\left[X,\left(U_{q}^{-}\right)_{-\beta}\right]=0 .
$$

On the other hand, for $\left(X_{1}, Y_{1}\right) \in\left(\tilde{U}_{q}^{+}\right)_{\beta} \times\left(\tilde{U}_{q}^{-}\right)_{-\beta}, Q_{\beta}\left(X_{1}, Y_{1}\right)=a \cdot\left\langle X_{1}, \phi\left(Y_{1}\right)\right\rangle$ for some non-zero element $a$ of $\mathbf{C}(q)$ (see (6.2.1)). Let $X_{2} \in\left(\widetilde{U}_{q}^{+}\right)_{\beta}$ be such that $\pi\left(X_{2}\right)=X$. By (6.1.1), (6.4.3) and (6.5.2), we see that $Q_{\beta}\left(X_{2}, Y_{2}\right)=0$ for any $Y_{2}$ $\in\left(\tilde{U}_{q}^{-}\right)_{-\beta}$. Hence $\left\langle X_{2}, X_{3}\right\rangle=0$ for any $X_{3} \in\left(\tilde{U}_{q}^{+}\right)_{\beta}$. Hence $X_{2} \in I_{\beta}^{+}$. Hence $X=0$.

Q. E. D.

6.6. Specialization at $q=1$. Let $\mathbf{A}=\mathbf{C}\left[q, q^{-1}\right]$ be the $\mathbf{C}$-subalgebra of $\mathbf{C}(q)$ generated by $q$ and $q^{-1}$. Let $\left(\mathscr{E}, \Pi=\left\{\alpha_{0}, \ldots, \alpha_{n}\right\}, p, \Gamma\right)$ be a lattice datum. Let $U_{\mathbf{A}}^{\sigma}$ be the A-subalgebra of $U_{q}^{o}$ generated by

$$
\sigma, K_{r},\left[K_{\gamma}\right]=\frac{K_{\gamma}-K_{r}^{-1}}{q-q^{-1}}(\gamma \in \Gamma), \quad E_{i}, F_{i}(0 \leq i \leq n) .
$$

Let $T_{\mathrm{A}}^{\sigma}$ (resp. $U_{\mathrm{A}}^{+}$, resp. $U_{\mathrm{A}}^{-}$) be the subalgebra of $T^{\sigma}$ (resp. $U_{q}^{+}$, resp. $U_{q}^{-}$) generated by $\sigma, K_{\gamma},\left[K_{\gamma}\right]$ (resp. $E_{i}$, resp. $\left.F_{i}\right)$. We note that, if $\{\gamma(r)(1 \leq r \leq \operatorname{dim} \mathscr{E})\}$ is a $\mathbb{Z}$ basis of $\Gamma$, then $\left\{\sigma^{a(0)} \prod_{r=1}^{\operatorname{dim} \delta} K_{\gamma(r)}^{a(r)}\left[K_{\gamma(r)}\right]^{b(r)} \mid \alpha(r)=0,1, b(r) \in \mathbf{Z}_{+}\right\}$is an A-basis of $T^{\sigma}$. Moreover there is an A-module isomorphism:

$$
U_{\mathbf{A}}^{+} \otimes_{\mathbf{A}} T_{\mathbf{A}}^{\sigma} \otimes_{\mathbf{A}} U_{\mathbf{A}}^{-} \rightarrow U_{\mathbf{A}}^{\sigma}
$$

$(X \otimes Z \otimes Y \rightarrow X Z Y)$.

Let $\mathbf{C}_{1}$ be a left $\mathbf{A}$-module such that $\operatorname{dim}_{\mathbf{C}} \mathbf{C}_{1}=1$ and $q$ acts as 1 . Define the $\mathbf{C}$ algebras $U_{\mathbf{C}}^{\sigma}, T_{\mathbf{C}}^{\sigma}, U_{\mathbf{C}}^{+}$and $U_{\mathbf{C}}^{-}$by $U_{\mathbf{A}}^{\sigma} \otimes_{\mathbf{A}} \mathbf{C}_{1}, T_{\mathbf{A}}^{\sigma} \otimes_{\mathbf{A}} \mathbf{C}_{1}, U_{\mathbf{A}}^{+} \otimes_{\mathbf{A}} \mathbf{C}_{1}$ and $U_{\mathbf{A}}^{-} \otimes_{\mathbf{A}} \mathbf{C}_{1}$. Then 
we have a $\mathbf{C}$-linear isomorphism:

$$
U_{\mathbf{C}}^{+} \otimes_{\mathbf{C}} T_{\mathbf{C}}^{g} \otimes_{\mathbf{C}} U_{\mathbf{C}}^{-} \rightarrow U_{\mathbf{C}}^{\sigma}
$$

$(X \otimes Z \otimes Y \rightarrow X Z Y)$. Define ' $U_{\mathrm{C}}^{\sigma}$ by the quotient $U_{\mathrm{C}}^{\sigma} / \mathscr{V}$ where $\mathscr{V}$ is the two-sided ideal of $U_{\mathbf{C}}^{\sigma}$ generated by all $K_{\gamma} \otimes_{\mathbf{A}} 1-1 \otimes_{\mathbf{A}} 1(\gamma \in \Gamma)$. Define $\pi_{1}: U_{\mathbf{A}}^{\sigma} \rightarrow^{\prime} U_{\mathrm{C}}^{\sigma}$ by $\pi_{1}(X)$ $=X \otimes_{\mathrm{A}} 1+\mathscr{V}$. Denote the elements $\pi_{1}(\sigma), \pi_{1}\left(\left[\boldsymbol{K}_{\gamma}\right]\right), \pi_{1}\left(E_{i}\right)$, and $\pi_{1}\left(F_{i}\right)$ by $\sigma, H_{\gamma}$, $E_{i}$ and $F_{i}$. It is clear that there is a unique Lie $\mathbf{C}$-superalgebra homomorphism $\tilde{\chi}$ : $\tilde{\mathscr{G}}(\mathscr{E}, \Pi, p) \rightarrow^{\prime} U_{\mathrm{C}}^{\sigma}$ such that $\tilde{\chi}\left(H_{\gamma}\right)=H_{\gamma}(\gamma \in \Gamma), \tilde{\chi}\left(E_{i}\right)=E_{i}$ and $\tilde{\chi}\left(F_{i}\right)=F_{i}$. Let $\mathscr{G}^{\Gamma}$ $=\mathscr{G}^{\Gamma}(\mathscr{E}, \Pi, p) \in I(\mathscr{E}, \Pi, p)$ be the admissible Lie superalgebra defined as $\tilde{\mathscr{G}}(\mathscr{E}, \Pi$, $p) / \operatorname{ker} \tilde{\chi}$. Then we have the Lie superalgebra monomorphism $\chi: \mathscr{G}^{\Gamma} \rightarrow^{\prime} U_{\mathrm{C}}^{\sigma}$ induced from $\tilde{\chi}$. Let $U\left(\mathscr{G}^{\Gamma}\right)$ be the universal enveloping superalgebra of $\mathscr{G}^{\Gamma}$. We denote by $\Xi$ the epimorphism $\Xi: U\left(\mathscr{G}^{\Gamma}\right) \rightarrow^{\prime} U_{\mathrm{C}}^{\sigma}$ such that $\Xi_{\mid \mathscr{g} \Gamma}=\chi$. It is well-known that the extension $U\left(\mathscr{G}^{\Gamma}\right)^{\sigma}$ of $U\left(\mathscr{G}^{\Gamma}\right)$ with $\sigma$ has a Hopf $\mathbf{C}$-algebra structure (see [Y1, Proposition 1.9.1]). It is clear that the extension $\Xi^{\sigma}$ of $\Xi$ with $\sigma$ is a Hopf $\mathbf{C}$-algebra epimorphism.

Lemma 6.6.1. $\Xi^{\sigma}$ is an isomorphism.

Proof. Let $\left\{X_{\theta}\right\}_{\theta \in \Theta}$ be a $\mathbb{C}$-basis of $\mathscr{G}^{\Gamma}$ where $\Theta$ is a totally ordered set. It is well-known that the PBW elements $\{1\} \cup\left\{X_{\theta_{1}} \cdots X_{\theta_{k}} \mid \theta_{1} \leq \cdots \leq \theta_{k}\right\}$ form a basis of $U\left(\mathscr{G}^{\Gamma}\right)$. We note that $1, \sigma, \sigma \Xi^{\sigma}\left(X_{\theta}\right), \Xi^{\sigma}\left(X_{\theta}\right)(\theta \in \Theta)$ are linearly independent. Observing the coefficients of the terms $\sigma^{a(1)} \otimes \sigma^{a(2)}, \sigma^{a(3)} \Xi^{\sigma}\left(X_{\theta}\right) \otimes \sigma^{a(4)}, \sigma^{a(5)} \Xi^{\sigma}\left(X_{\theta_{1}}\right) \otimes$ $\sigma^{a(6)} \Xi^{\sigma}\left(X_{\theta_{2}} \cdots X_{\theta_{k}}\right)\left(\theta_{1} \leq \theta_{2} \leq \cdots \leq \theta_{k}\right)(a(j)=0,1)$ of $\Xi^{\sigma} \otimes \Xi^{\sigma}(\Delta(Y))=\Delta\left(\Xi^{\sigma}(Y)\right)$ of $Y \in U\left(\mathscr{G}^{\Gamma}\right)^{\sigma}$, we see that ker $\Xi^{\sigma}=\{0\}$.

Q. E. D.

Put $\mathcal{N}^{I^{ \pm}}=\Psi\left[\tilde{\mathscr{G}}, \mathscr{G}^{\Gamma}\right]\left(\tilde{\mathscr{N}}^{ \pm}\right)$. By Lemma 6.6.1, we have

$$
\operatorname{dim}_{\mathbf{C}(q)}\left(U_{q}^{ \pm}\right)_{ \pm \alpha}=\operatorname{dim}_{\mathbf{C}} U\left(\mathscr{N}^{\Gamma \pm}\right)_{ \pm \alpha} \geq \operatorname{dim}_{\mathbf{C}} U\left(\mathscr{N}^{ \pm}\right)_{ \pm \alpha} \quad\left(\alpha \in P_{+}\right)
$$

where $U\left(\mathscr{N}^{ \pm}\right)_{ \pm \alpha}=\left\{W \in U\left(\mathcal{N}^{ \pm}\right) \mid[H, W]= \pm \alpha(H) W(H \in \mathscr{H})\right\}$ and $U\left(\mathscr{N}^{\Gamma \pm}\right)_{ \pm \alpha}$ $=\left\{W \in U\left(\mathscr{N}^{\Gamma^{ \pm}}\right)_{ \pm \alpha} \mid[H, W]= \pm \alpha(H) W(H \in \mathscr{H})\right\}$.

6.7. For $\alpha \in P$, put $\left(U_{q}^{\sigma}\right)_{\alpha}=\left\{X \in U_{q}^{\sigma} \mid K_{\gamma} X K_{\gamma}^{-1}=q^{(\alpha, \gamma)} X(\gamma \in \Gamma)\right\}$. For $X_{\alpha} \in\left(U_{q}^{\sigma}\right)_{\alpha}$, $X_{\beta} \in\left(U_{q}^{\sigma}\right)_{\beta}$, we put:

$$
\llbracket X_{\alpha}, X_{\beta} \rrbracket=X_{\alpha} X_{\beta}-(-1)^{p(\alpha) p(\beta)} q^{-(\alpha, \beta)} X_{\beta} X_{\alpha} .
$$

Let $[n]=\frac{q^{n}-q^{-n}}{q-q^{-1}}$.

We say that $(\mathscr{E}, \Pi, p)$ is of affine $\mathrm{ABCDFG}$ type if $(\mathscr{E}, \Pi, p) \in \mathrm{ABCD}(N)$ for some $N \geq 1$, or $(\mathscr{E}, \Pi, p) \in \operatorname{Data}\left(\mathrm{F}(4)^{(1)}\right) \cup \operatorname{Data}\left(\mathrm{G}(3)^{(1)}\right)$. For $(\mathscr{E}, \Pi, p)$ of affine ABCDFG type, we fix a lattice datum $(\mathscr{E}, \Pi, p, \Gamma)$ as follows: 


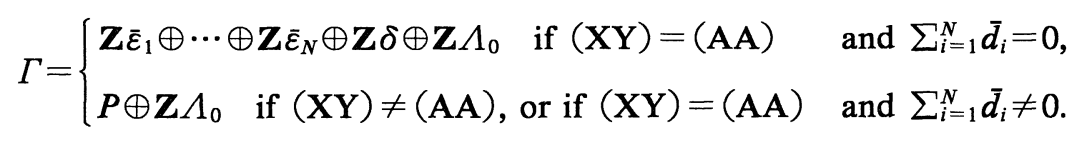

We also say that $(\mathscr{E}, \Pi, p, \Gamma)$ is of affine ABCDFG type.

By using Proposition 6.5.1, we have:

Proposition 6.7.1. Let $(\mathscr{E}, \Pi, p, \Gamma)$ be a lattice datum of affine ABCDFG type. Then, in $U_{q}^{\sigma}(\mathscr{E}, \Pi, p, \Gamma)$, the following equalities (QS4) (a) $(1 \leq a \leq 17)$ satisfied by $E_{r}$ 's hold:

$(\mathrm{QS} 4)(1) \quad\left[E_{i}, E_{j}\right]=0$ if $\left(\alpha_{i}, \alpha_{j}\right)=0(i \neq j)$

$(\mathrm{QS4})(2) \quad\left[E_{i}, E_{i}\right]=0$ if $\stackrel{i}{\otimes}$,

$(\mathrm{QS4})(3) \llbracket E_{i}, \llbracket E_{i}, \ldots, \llbracket E_{i}, E_{j} \rrbracket \ldots \rrbracket \rrbracket=0\left(E_{i}\right.$ appears $1-\frac{2\left(\alpha_{i}, \alpha_{j}\right)}{\left(\alpha_{i}, \alpha_{i}\right)}$ times $)$

$$
\text { if }\left(\alpha_{i}, \alpha_{i}\right) \neq 0 \text { and }(-1)^{\left\{p\left(\alpha_{i}\right) \frac{2\left(\alpha_{i}, \alpha_{i}\right)}{\left(\alpha_{i}, \alpha_{i}\right)}\right\}}=1 \text {, }
$$

(QS4) (4) $\quad\left[\llbracket \llbracket E_{i}, E_{j} \rrbracket, E_{k} \rrbracket, E_{j}\right]=0$

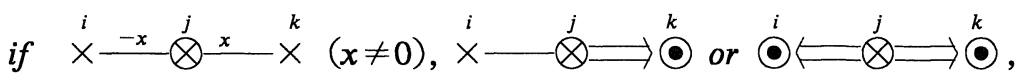

$(\mathrm{QS} 4)(5) \quad\left[\llbracket \llbracket E_{i}, E_{j} \rrbracket, \llbracket \llbracket E_{i}, E_{j} \rrbracket, E_{k} \rrbracket \rrbracket, E_{j}\right]=0$

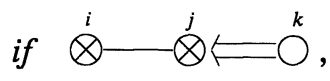

(QS4) (6) $\quad\left[\llbracket \llbracket \llbracket \llbracket \llbracket E_{i}, E_{j} \rrbracket, E_{k} \rrbracket, E_{l} \rrbracket, E_{k} \rrbracket, E_{j} \rrbracket, E_{k}\right]=0$

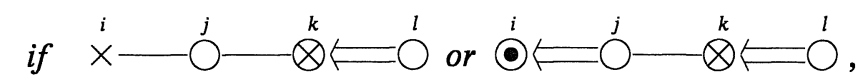

$(\mathrm{QS} 4)(7) \quad(-1)^{p\left(\alpha_{i}\right) p\left(\alpha_{k}\right)}\left[\left(\alpha_{i}, \alpha_{k}\right)\right] \llbracket \llbracket E_{i}, E_{j} \rrbracket, E_{k} \rrbracket$

$$
\begin{array}{r}
=(-1)^{p\left(\alpha_{i}\right) p\left(\alpha_{j}\right)}\left[\left(\alpha_{i}, \alpha_{j}\right)\right]\left[\llbracket E_{i}, E_{k} \rrbracket, E_{j} \rrbracket\right. \\
\text { if }\left(\alpha_{i}, \alpha_{j}\right) \neq 0,\left(\alpha_{j}, \alpha_{k}\right) \neq 0,\left(\alpha_{k}, \alpha_{i}\right) \neq 0, \\
\left(\alpha_{i}, \alpha_{j}\right)+\left(\alpha_{j}, \alpha_{k}\right)+\left(\alpha_{k}, \alpha_{i}\right)=0 \\
\text { and } p\left(\alpha_{i}\right) p\left(\alpha_{j}\right)+p\left(\alpha_{j}\right) p\left(\alpha_{k}\right)+p\left(\alpha_{k}\right) p\left(\alpha_{i}\right)=\overline{1},
\end{array}
$$

(QS4) (8) $\llbracket \llbracket \llbracket E_{j}, E_{i} \rrbracket, \llbracket E_{j}, E_{k} \rrbracket \rrbracket, \llbracket E_{j}, E_{l} \rrbracket \rrbracket=\llbracket \llbracket \llbracket E_{j}, E_{i} \rrbracket, \llbracket E_{j}, E_{l} \rrbracket \rrbracket, \llbracket E_{j}, E_{k} \rrbracket \rrbracket$

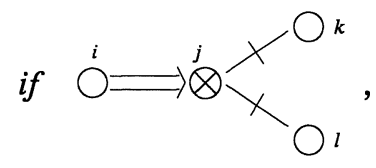


(QS4) (9) $\quad\left[\llbracket \llbracket E_{k}, \llbracket E_{l}, \llbracket E_{k}, E_{j} \rrbracket \rrbracket \rrbracket, \llbracket E_{k}, \llbracket E_{l}, \llbracket E_{k}, \llbracket E_{j}, E_{i} \rrbracket \rrbracket \rrbracket \rrbracket, E_{j}\right]$

$=[2] \llbracket \llbracket E_{k}, E_{j} \rrbracket, \llbracket \llbracket E_{l}, \llbracket E_{k}, E_{j} \rrbracket \rrbracket, \llbracket E_{k}, \llbracket E_{l}, \llbracket E_{k}, \llbracket E_{j}, E_{i} \rrbracket \rrbracket \rrbracket \rrbracket \rrbracket$

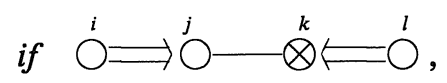

(QS4) (10) $\llbracket E_{j}, \llbracket E_{k}, \llbracket E_{j}, \llbracket E_{k}, E_{i} \rrbracket \rrbracket \rrbracket=\llbracket E_{k}, \llbracket E_{j}, \llbracket E_{k}, \llbracket E_{j}, E_{i} \rrbracket \rrbracket \rrbracket$

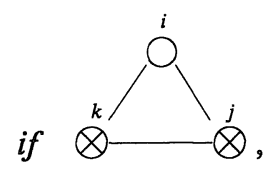

$(\mathrm{QS} 4)(11) \quad \llbracket \llbracket E_{k}, E_{j} \rrbracket, \llbracket \llbracket E_{k}, E_{j} \rrbracket, \llbracket \llbracket E_{k}, E_{j} \rrbracket, E_{i} \rrbracket \rrbracket \rrbracket$

$=\left(1-(-1)^{p\left(\alpha_{k}\right)}[2]\right) \llbracket \llbracket \llbracket E_{k}, E_{j} \rrbracket, \llbracket E_{k}, \llbracket E_{k}, \llbracket E_{j}, E_{i} \rrbracket \rrbracket \rrbracket \rrbracket, E_{j} \rrbracket$

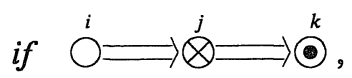

$(\mathrm{QS} 4)(12) \quad\left[\llbracket \llbracket \llbracket \llbracket \llbracket \llbracket \llbracket \llbracket E_{i}, E_{j} \rrbracket, E_{k} \rrbracket, E_{l} \rrbracket, E_{k} \rrbracket, E_{j} \rrbracket, E_{k} \rrbracket, E_{l} \rrbracket, E_{k} \rrbracket, E_{j} \rrbracket, E_{k}\right]=0$

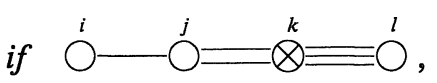

$(\mathrm{QS} 4)(13) \quad \llbracket \llbracket \llbracket \llbracket E_{l}, E_{k} \rrbracket, E_{j} \rrbracket, E_{i} \rrbracket, E_{k} \rrbracket, E_{j} \rrbracket=[2] \llbracket \llbracket \llbracket \llbracket\left[E_{l}, E_{k} \rrbracket, E_{j} \rrbracket, E_{i} \rrbracket, E_{j} \rrbracket, E_{k} \rrbracket\right.$

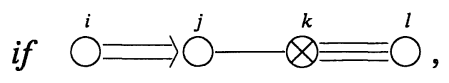

(QS4) (14) $\quad\left[\llbracket \llbracket E_{i}, E_{j} \rrbracket, \llbracket \llbracket E_{i}, E_{j} \rrbracket, \llbracket \llbracket E_{i}, E_{j} \rrbracket, E_{k} \rrbracket \rrbracket \rrbracket, E_{j}\right]=0$

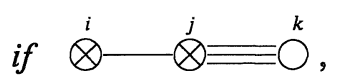

(QS4) (15) $\llbracket E_{j}, \llbracket E_{k}, \llbracket E_{k}, \llbracket E_{j}, E_{i} \rrbracket \rrbracket \rrbracket=\llbracket E_{k}, \llbracket E_{j}, \llbracket E_{k}, \llbracket E_{j}, E_{i} \rrbracket \rrbracket \rrbracket$

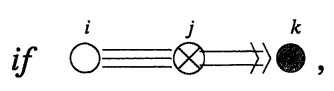

$(\mathrm{QS} 4)(16) \quad[2] \llbracket \llbracket \llbracket \llbracket \llbracket E_{l}, E_{k} \rrbracket, E_{j} \rrbracket, E_{i} \rrbracket, E_{k} \rrbracket, E_{j} \rrbracket$

$=[3] \llbracket \llbracket \llbracket \llbracket \llbracket E_{l}, E_{k} \rrbracket, E_{j} \rrbracket, E_{i} \rrbracket, E_{j} \rrbracket, E_{k} \rrbracket$

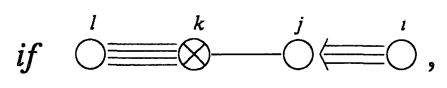

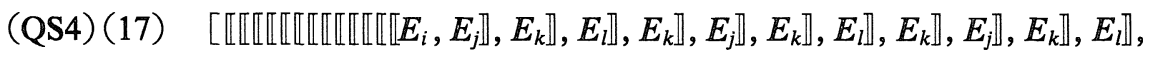
$\left.E_{k} \rrbracket, E_{j} \rrbracket, E_{k}\right]=0$ 


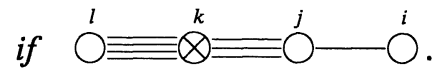

Proof. Let $\mathscr{X}(a)$ denote the left hand side minus the right hand side of the equality in (QS4) (a). To prove the proposition, it suffices to show

$$
\left[\mathscr{X}(a), F_{r}\right]=0 \quad \text { for any } r=i, j, k, l \text {. }
$$

(see Proposition 6.5.1). By direct calculation, whose examples will be given in 6.9-6.11, we get (6.7.1).

Q. E. D.

By using $\phi$, we have:

Lemma 6.7.2. Let $(\mathscr{E}, \Pi, p, \Gamma)$ be a lattice datum of affine ABCDFG type. Then, in $U_{q}^{\sigma}(\mathscr{E}, \Pi, p, \Gamma)$, the following equalities (QS5) $(a)(1 \leq a \leq 17)$ satidfied by $F_{r}$ 's hold:

(QS5) (a) The same equations with $F_{r}$ 's in place of $E_{r}$ 's in (QS4) (a).

6.8.

Proposition 6.8.1. Let $\left(\mathscr{E}, \Pi=\left\{\alpha_{0}, \ldots, \alpha_{n}\right\}, p, \Gamma\right)$ be a lattice datum of affine ABCDFG type. Assume that $\mathscr{G}(\mathscr{E}, \Pi, p)=\mathscr{G}^{\natural}(\mathscr{E}, \Pi, p)$. Then we have:

(1) $\mathscr{G}(\mathscr{E}, \Pi, p)=\mathscr{G}^{\Gamma}(\mathscr{E}, \Pi, p)$.

(2) $U_{q}^{+}$can also be defined by the generators $E_{r}(0 \leq r \leq n)$ and the relations in (QS4).

Proof. (1) This follows immediately by Theorem 4.1.1, Theorem 5.2.1, Theorem 5.3.1, Proposition 6.7.1 and Lemma 6.7.2, and by comparing relations in (S1-5) and (QS1-5).

(2) We first note that $\mathcal{N}^{+}$can also be defined by generators $E_{r}(0 \leq r \leq n)$ and the relations in (S4) (a) $(1 \leq a \leq 17)$ (see Step 1 of the proof of Theorem 4.1.1). Let ${ }^{b} U_{q}^{+}$be the $\mathbf{C}(q)$-algebra defined by generators $E_{i}(0 \leq i \leq n)$ and the relations obtained by the equations $(\mathrm{QS} 4)(a)(1 \leq a \leq 17)$. Then we have:

$$
\operatorname{dim}_{\mathbf{C}} U\left(\mathcal{N}^{+}\right)_{\alpha} \geq \operatorname{dim}_{\mathbf{C}(q)}\left({ }^{b} U_{q}^{+}\right)_{\alpha} \geq \operatorname{dim}_{\mathbf{C}(q)}\left(U_{q}^{+}\right)_{\alpha} \quad\left(\alpha \in P_{+}\right) .
$$

By (6.6.3), we have $\operatorname{dim}_{\mathrm{C}(q)}\left({ }^{b} U_{q}^{+}\right)_{\alpha}=\operatorname{dim}_{\mathrm{C}(q)}\left(U_{q}^{+}\right)_{\alpha}$, which implies the statement of (2).

Q. E. D.

We say that $(\mathscr{E}, \Pi, p, \Gamma)$ of affine ABCDFG type is natural if $\mathscr{G}^{\natural}(\mathscr{E}, \Pi, p)=$ $\mathscr{G}(\mathscr{E}, \Pi, p)$. In Theorem 3.5.1, we classified the data of natural affine ABCD type. 
We note that any $(\mathscr{E}, \Pi, p) \in \operatorname{Data}\left(\mathrm{F}(4)^{(1)}\right) \cup \operatorname{Data}\left(\mathrm{G}(3)^{(1)}\right)$ is natural. Let $U_{q}=$ $U_{q}\left(\mathscr{E}, \Pi,=\left\{\alpha_{0}, \ldots, \alpha_{n}\right\}, p, \Gamma\right)$ be a $\mathbf{C}(q)$-subalgebra of $U_{q}^{\sigma}(\mathscr{E}, \Pi, p, \Gamma)$ generated by $K_{\gamma}(\gamma \in \Gamma), E_{i}, F_{i}(0 \leq i \leq n)$. Then $U_{q}^{\sigma}$ is the extension of $U_{q}$ with $\sigma$. As an immediate consequence of Proposition 6.8.1, we have:

Theorem 6.8.2. Let $\left(\mathscr{E}, \Pi=\left\{\alpha_{0}, \ldots, \alpha_{n}\right\}, p, \Gamma\right)$ be a lattice datum of natural affine ABCDFG type. Then $\mathrm{C}(q)$-algebra $U_{q}(\mathscr{E}, \Pi, p, \Gamma)$ can also be defined by generators $K_{r}(\gamma \in \Gamma), E_{i}, F_{i}(0 \leq i \leq n)$ and relations:

$$
\text { (QS1), (QS2), (QS3), (QS4) (a) }(1 \leq a \leq 17),(\mathrm{QS5})(a)(1 \leq a \leq 17) \text {. }
$$

Remark 6.8.3. We note that, for a non-super $(\mathscr{E}, \Pi, p, \Gamma), U_{q}$ coincides with the Drinfeld $[\mathrm{D} 1]$ and Jimbo $[\mathrm{J}]$ quantized universal enveloping algebra (see $[\mathrm{Y} 1$, Corollary 2.10.1] and [L1, Corollary 33.1.5]).

6.9. Useful formulas. For $a \in \mathbf{C}(q)$, we put:

$$
[X, Y]_{a}=X Y-(-1)^{p(X)_{p}(Y)} a Y X \quad\left(X, Y \in U_{q}\right)
$$

Then we have

$$
\left[[X, Y]_{a}, Z\right]_{b}=\left[X,[Y, Z]_{c}\right]_{a b c}-1+(-1)^{p(Y)_{p}(Z)} c\left[[X, Z]_{b c}-1, Y\right]_{a c}{ }^{-1},
$$

and

$$
\left[X,[Y, Z]_{a}\right]_{b}=\left[[X, Y]_{c}, Z\right]_{a b c}-1+(-1)^{p(X) p(Y)} c\left[Y,[X, Z]_{b c}-1\right]_{a c}-1
$$

Hence, for $X_{\nu} \in\left(U_{q}\right)_{\nu}, X_{\mu} \in\left(U_{q}\right)_{\mu}$ and $X_{\eta} \in\left(U_{q}\right)_{\eta}$, we have

$$
\llbracket \llbracket X_{\nu}, X_{\mu} \rrbracket, X_{\eta} \rrbracket=\llbracket X_{\nu}, \llbracket X_{\mu}, X_{\eta} \rrbracket \rrbracket+(-1)^{p(\mu) p(\eta)} q^{-(\mu, \eta)}\left[\llbracket X_{\nu}, X_{\eta} \rrbracket, X_{\mu}\right]_{q}^{(\mu, \eta-\nu)} \text {, }
$$

and

(6.9.4) $\llbracket X_{\nu}, \llbracket X_{\mu}, X_{\eta} \rrbracket \rrbracket=\llbracket \llbracket X_{\nu}, X_{\mu} \rrbracket, X_{\eta} \rrbracket+(-1)^{p(\mu) p(\nu)} q^{-(\mu, \nu)}\left[X_{\mu}, \llbracket X_{\nu}, X_{\eta} \rrbracket\right]_{q}^{(\mu, \nu-\eta)}$.

For $\left(\mathscr{E}, \Pi=\left\{\alpha_{0}, \ldots, \alpha_{n}\right\}, p, \Gamma\right)$, put $K_{i}=K_{\alpha_{i}}$. We note

$$
\llbracket E_{i}, F_{j} K_{j}^{-1} \rrbracket=\delta_{i j} \frac{1-K_{i}^{-2}}{q-q^{-1}}, \quad \llbracket K_{j} F_{j}, E_{i} \rrbracket=-\delta_{i j}(-1)^{p\left(\alpha_{i}\right) p\left(\alpha_{j}\right)} \frac{K_{i}^{2}-1}{q-q^{-1}} .
$$

Then, by (6.9.3-5), we have

$$
\llbracket \llbracket E_{i}, X_{\mu} \rrbracket, F_{j} K_{j}^{-1} \rrbracket=\llbracket E_{i}, \llbracket X_{\mu}, F_{j} K_{j}^{-1} \rrbracket \rrbracket+\delta_{i j}(-1)^{p(\mu) p\left(\alpha_{j}\right)}\left[\left(\mu, \alpha_{i}\right)\right] X_{\mu}
$$


and

$$
\llbracket K_{j} F_{j}, \llbracket X_{\mu}, E_{i} \rrbracket \rrbracket=\llbracket \llbracket K_{j} F_{j}, X_{\mu} \rrbracket, E_{i} \rrbracket+\delta_{i j}(-1)^{p(\mu) p\left(\alpha_{j}\right)+p\left(\alpha_{j}\right)}\left[\left(\mu, \alpha_{i}\right)\right] X_{\mu}
$$

6.10. Here we show how to check the relation (QS4) (9). We replace the letters $i, j, k, l$ with $0,1,2,3$. We assume $\left(\alpha_{1}, \alpha_{1}\right)=-2$. Put $E_{\ldots d c b a}=\llbracket \ldots \llbracket E_{d}, \llbracket E_{c}, \llbracket E_{b}$, $E_{a} \rrbracket \rrbracket$..... Then the element $\mathscr{X}=\mathscr{X}(9)$ is rewritten as:

$$
-\llbracket \llbracket E_{2321}, E_{23210} \rrbracket, E_{1} \rrbracket+\left(q+q^{-1}\right) \llbracket E_{21} \llbracket E_{321}, E_{23210} \rrbracket \rrbracket
$$

We note that, to show $\left[\mathscr{X}, F_{i}\right]=0(i=0,1,2,3)$, it suffices to show $\llbracket \mathscr{X}, F_{i} K_{i}^{-1} \rrbracket=$ 0 or $\llbracket K_{i} F_{i}, \mathscr{X} \rrbracket=0$. First we show $\llbracket \mathscr{X}, F_{3} K_{3}^{-1} \rrbracket=0$. In following equalities, $L H S \sim$ $R H S$ means $L H S=a \cdot R H S$ for some $a \in \mathbf{C}(q) \backslash\{0\}$. By (6.9.6), we have

$$
\begin{aligned}
\llbracket E_{2321}, & F_{3} K_{3}^{-1} \rrbracket \\
& \sim \llbracket E_{2}, \llbracket E_{321}, F_{3} K_{3}^{-1} \rrbracket \rrbracket \\
& \sim \llbracket E_{2}, E_{21} \rrbracket \\
& =0 \quad\left(\text { by } E_{2}^{2}=0(\sec (\operatorname{QS} 4)(2))\right) .
\end{aligned}
$$

Similarly we have $\llbracket E_{23210}, F_{3} K_{3}^{-1} \rrbracket=0$. Hence we have

$$
\llbracket \llbracket \llbracket E_{2321}, E_{23210} \rrbracket, E_{1} \rrbracket, F_{3} K_{3}^{-1 \rrbracket}=0 .
$$

Moreover we have

$$
\begin{aligned}
\llbracket \llbracket E_{21}, & \llbracket E_{321}, E_{23210} \rrbracket \rrbracket, F_{3} K_{3}^{-1} \rrbracket \\
& \sim \llbracket E_{21}, \llbracket E_{21}, E_{23210} \rrbracket \rrbracket \quad(\text { by }(6.9 .3)) \\
& =0 \quad\left(\text { by } E_{21}^{2}=0\right) .
\end{aligned}
$$

Hence we have $\llbracket \mathscr{X}, F_{3} K_{3}^{-1} \rrbracket=0$.

Next we show $\llbracket \mathscr{X}, F_{2} K_{2}^{-1} \rrbracket=0$. Using (6.9.3-7), we have

$$
\begin{aligned}
& \llbracket E_{2321}, F_{2} K_{2}^{-1} \rrbracket=E_{321}, \quad \llbracket E_{23210}, F_{2} K_{2}^{-1} \rrbracket=E_{3210}, \\
& \llbracket \llbracket E_{2321}, E_{23210} \rrbracket, F_{2} K_{2}^{-1} \rrbracket=\llbracket E_{2321}, E_{3210} \rrbracket+q^{-1}\left[E_{321}, E_{23210}\right]_{q}^{(1-0)} \\
& =-q^{-2}\left[E_{23210}, E_{321}\right]_{q^{(2+1)}}+q^{-1}\left[E_{321}, E_{23210}\right]_{q^{(1-0)}}\left(\text { since } E_{321}^{2}=0\right) \\
& =\left(q+q^{-1}\right) \llbracket E_{321}, E_{23210} \rrbracket \text {, } \\
& \llbracket \llbracket E_{321}, E_{23210} \rrbracket, F_{2} K_{2}^{-1} \rrbracket=0 \text { and } \llbracket E_{21}, F_{2} K_{2}^{-1} \rrbracket=E_{1} \text {. }
\end{aligned}
$$

Using these, we have 
$\llbracket \mathscr{X}, F_{2} K_{2}^{-1} \rrbracket$

$=-q\left[\left(q+q^{-1}\right) \llbracket E_{321}, E_{23210} \rrbracket, E_{1}\right]_{q}(-1-2)-\left(q+q^{-1}\right) q^{-2}\left[E_{1}, \llbracket E_{321}, E_{23210} \rrbracket\right]_{q}(2+1)$ $=0$.

Next we show that $\llbracket K_{1} F_{1}, \mathscr{X} \rrbracket=0$. Using (6.9.3-7), it follows that $\llbracket K_{1} F_{1}, E_{21} \rrbracket$ $=E_{2}, \llbracket K_{1} F_{1}, E_{321} \rrbracket=E_{32}, \llbracket K_{1} F_{1}, E_{23210} \rrbracket=0$ and $\llbracket K_{1} F_{1}, E_{2321} \rrbracket=q\left[E_{2}, E_{32}\right]$. Since $\llbracket E_{2}, E_{23210} \rrbracket=\llbracket E_{32}, E_{23210} \rrbracket=0$ (by $E_{2}^{2}=E_{32}^{2}=0$ ), we have $\llbracket K_{1} F_{1}, \llbracket E_{2321}, E_{23210} \rrbracket \rrbracket=0$. We also have $\llbracket K_{1} F_{1}, \llbracket E_{321}, E_{23210} \rrbracket \rrbracket=0$. Hence we have

$$
\begin{aligned}
& \llbracket K_{1} F_{1}, \mathscr{X} \rrbracket \\
& \quad=-\left(q+q^{-1}\right) \llbracket E_{2321}, E_{23210} \rrbracket+\left(q+q^{-1}\right) \llbracket E_{2}, \llbracket E_{321}, E_{23210} \rrbracket \rrbracket \\
& \quad=0 .
\end{aligned}
$$

Finally we show that $\llbracket K_{0} F_{0}, \mathscr{X} \rrbracket=0$. Using (6.9.3-7), we have $\llbracket K_{0} F_{0}, E_{23210} \rrbracket$ $=\left(q+q^{-1}\right) E_{2321}$. We note

$$
\llbracket E_{2321}, E_{1} \rrbracket=q\left[E_{21}, E_{321}\right]=q\left(1-q^{2}\right) E_{21} E_{321}
$$

since $\llbracket E_{321}, E_{21} \rrbracket=0$. Then we have

$$
\begin{aligned}
\llbracket K_{0} F_{0}, \mathscr{X} \rrbracket \\
\sim-\llbracket q^{2}\left[E_{2321}, E_{2321}\right]_{q^{-2}}, E_{1} \rrbracket+\left(q+q^{-1}\right) q^{4}\left[E_{21},\left[E_{321}, E_{2321}\right]_{q^{-3}}\right]_{q^{-1}} \\
=-q^{2}\left(1-q^{-2}\right)\left(q\left(1-q^{2}\right)\right)\left(E_{2321} E_{21} E_{321}+E_{21} E_{321} E_{2321}\right) \\
\quad+\left(q+q^{-1}\right) q^{4}\left(\left[E_{21},\left(1-q^{-3+1}\right) E_{321} E_{2321}\right]_{q^{-1}}\right) \\
=\left(q^{2}-q^{-2}\right)\left(-q^{3}\left(1-q^{2}\right)+q^{3}\left(1-q^{2}\right)\right) E_{21} E_{321} E_{2321} \\
=0
\end{aligned}
$$

where we note that $\llbracket E_{2321}, E_{321} \rrbracket=0$ (by $E_{321}^{2}=0$ ) and $\llbracket E_{2321}, E_{21} \rrbracket=0$.

From the above calculation, we obtain $\left[\mathscr{X}, F_{i}\right]=0$ for all $i=0,1,2,3$, as desired.

6.11. Here we show how to check the relation (QS4) (17). We replace the letters $i, j, k, l$ with $0,1,2,3$. We assume $\left(\alpha_{1}, \alpha_{1}\right)=6$. Put $E_{a b c d \ldots}^{\vee}=\llbracket \ldots \llbracket \llbracket \llbracket \llbracket E_{a}, E_{b} \rrbracket, E_{c} \rrbracket$, $E_{d} \rrbracket \ldots \rrbracket$. Then the element $\mathscr{Y}=\mathscr{X}(17)$ is rewritten as:

\section{$E_{012321232123212}^{\vee}$}

As in 6.7, we show that $\llbracket \mathscr{Y}, F_{i} K_{i}^{-1} \rrbracket=0$ or $\llbracket K_{i} F_{i}, \mathscr{Y} \rrbracket=0$ for $i=0,1,2,3$. It is clear from (6.9.7) and $E_{2}^{2}=\left[E_{0}, E_{2}\right]=0$ that $\llbracket K_{1} F_{1}, \mathscr{Y} \rrbracket=\llbracket K_{3} F_{3}, \mathscr{Y} \rrbracket=0$. We note that

$\llbracket E_{012}^{\vee}, E_{1} \rrbracket=0$ (by $\left.(\mathrm{QS} 4)(3)\right)$, $E_{013}^{\vee}=0$, 


$$
\begin{aligned}
& E_{01231}^{\vee}=\llbracket \llbracket E_{012}^{\vee}, E_{1} \rrbracket, E_{3} \rrbracket=0, \\
& E_{0123213}^{\vee}=E_{0123231}^{\vee}=\llbracket \llbracket \llbracket E_{01}^{\vee}, E_{23}^{\vee} \rrbracket, E_{23}^{\vee} \rrbracket, E_{1} \rrbracket=\llbracket 0, E_{1} \rrbracket=0\left(\text { by }\left(E_{23}^{\vee}\right)^{2}=0\right) .
\end{aligned}
$$

Similarly we have

$$
\begin{aligned}
& E_{012321231}^{\vee}=E_{012321213}^{\vee}=\llbracket \llbracket \llbracket \llbracket E_{0123}^{\vee}, E_{21}^{\vee} \rrbracket, E_{2} \rrbracket, E_{1} \rrbracket, E_{3} \rrbracket \\
& =\llbracket \llbracket \llbracket E_{0123}^{\vee}, E_{21}^{\vee} \rrbracket, E_{21}^{\vee} \rrbracket, E_{3} \rrbracket=\llbracket 0, E_{3} \rrbracket=0
\end{aligned}
$$

by $\llbracket E_{012}^{\vee}, E_{1} \rrbracket=0, \llbracket E_{21}^{\vee}, E_{1} \rrbracket=0$ and $\left(E_{21}^{\vee}\right)^{2}=0$. Similarly we have

$$
E_{01232123213}^{\vee}=E_{01232123231}^{\vee}=\llbracket \llbracket \llbracket E_{012321}^{\vee}, E_{23}^{\vee} \rrbracket, E_{23}^{\vee} \rrbracket, E_{1} \rrbracket=\llbracket 0, E_{1} \rrbracket=0
$$

where we note that $\llbracket E_{01232}^{\vee}, E_{3} \rrbracket=\llbracket \llbracket\left[E_{01}^{\vee}, E_{23}^{\vee} \rrbracket, E_{23}^{\vee} \rrbracket=0\right.$ (by $\left.\left(E_{23}^{\vee}\right)^{2}=0\right)$. By (6.9.7), and by the above equations, letting $\mu=\alpha_{0}+4 \alpha_{1}+6 \alpha_{2}+3 \alpha_{3}$, we have

$$
\llbracket K_{2} F_{2}, \mathscr{Y} \rrbracket=\left[\left(\mu, \alpha_{2}\right)\right] E_{01232123212321}^{\vee}=0 .
$$

Finally we show that $\llbracket \mathscr{Y}, F_{0} K_{0}^{-1} \rrbracket=0$. We have

$$
\begin{aligned}
\mathscr{Y}= & E_{012321232123212}^{\vee} \\
& =\llbracket \llbracket \llbracket \llbracket \llbracket \llbracket E_{012}^{\vee}, E_{32}^{\vee} \rrbracket, E_{12}^{\vee} \rrbracket, E_{32}^{\vee} \rrbracket, E_{12}^{\vee} \rrbracket, E_{32}^{\vee} \rrbracket, E_{12}^{\vee} \rrbracket \\
& =\llbracket \llbracket \llbracket\left[E_{012}^{\vee}, E_{32}^{\vee} \rrbracket, \llbracket E_{12}^{\vee}, E_{32}^{\vee} \rrbracket\right], \llbracket\left[E_{12}^{\vee}, E_{32}^{\vee} \rrbracket\right], E_{12}^{\vee} \rrbracket .
\end{aligned}
$$

since $E_{2}^{2}=\left(E_{23}^{\vee}\right)^{2}=\left(E_{12}^{\vee}\right)^{2}=0$. By (6.9.3) and (6.9.6),

$$
\llbracket \mathscr{Y}, F_{0} K_{0}^{-1} \rrbracket \sim \llbracket E_{12}^{\vee},\left(\llbracket E_{12}^{\vee}, E_{32}^{\vee} \rrbracket\right)^{3} \rrbracket=0
$$

From the above calculation, we obtain $\left[\mathscr{Y}, F_{i}\right]=0$ for all $i=0,1,2,3$, as desired.

\subsection{2}

Proposition 6.12.1. Let $\left(\mathscr{E}, \Pi=\left\{\alpha_{0}, \ldots, \alpha_{n}\right\}, p, \Gamma\right)$ be a lattice datum. Let ${ }^{\sharp} U_{q}$ $={ }^{\#} U_{q}(\mathscr{E}, \Pi, p, \Gamma)$ be the $\mathbf{Z}_{2}$-graded $\mathbf{C}(q)$-algebra (with 1) satisfying the following conditions:

(a) The extension ${ }^{\sharp} U_{q}^{\sigma}$ of ${ }^{\sharp} U_{q}$ with $\sigma$ has a Hopf algebra structure.

(b) There is a $\mathbf{Z}_{2}$-graded $\mathbf{C}(q)$-algebra epimorphism ${ }^{M \#} \Psi_{q}=\Psi_{q}\left[\widetilde{U}_{q},{ }^{\sharp} U_{q}\right]: \widetilde{U}_{q}$ $\rightarrow U_{q}$ such that $\left({ }^{M \#} \Psi_{q}\right)_{\mid T}$ is injective.

(c) The extension ${ }^{M} \Psi_{q}^{\sigma}$ : $\widetilde{U}_{q}^{\sigma} \rightarrow^{\sharp} U_{q}^{\sigma}$ is a Hopf algebra epimorhism.

Then there is a $\mathbf{C}(q)$-algebra epimorphism ${ }^{\sharp m} \Psi_{q}=\Psi_{q}\left[{ }^{\sharp} U_{q}, U_{q}\right]:{ }^{\sharp} U_{q} \rightarrow U_{q}$ such that 
$\Psi_{q}\left[{ }^{\sharp} U_{q}, U_{q}\right] \circ \Psi_{q}\left[\widetilde{U}_{q},{ }^{\sharp} U_{q}\right]$ is the canonical map and the extension ${ }^{\sharp m} \Psi_{q}^{\sigma}$ of ${ }^{\sharp m} \Psi_{q}$ with $\sigma$ is a Hopf algebra epimorphism.

Proof. We denote ${ }^{M \#} \Psi_{q}(T),{ }^{M \#} \Psi_{q}\left(\widetilde{U}_{q}^{+}\right)$and ${ }^{M \#} \Psi_{q}\left(\tilde{U}_{q}^{-}\right)$by $T$, ${ }^{\sharp} U_{q}^{+}$and ${ }^{\sharp} U_{q}^{-}$. We note that ${ }^{M \#} \Psi_{q}\left(E_{i}\right) \neq 0$ and ${ }^{M \#} \Psi_{q}\left(F_{i}\right) \neq 0$ since $\left[E_{i}, F_{i}\right] \in T \backslash\{0\}$. By exactly the same argument as in the proof of $[\mathbf{R}$, Proposition 2], we have a $\mathbf{C}(q)$-linear isomorphism:

$$
{ }^{\#} U_{q}^{+} \otimes T^{\sigma} \otimes \# U_{q}^{-} \rightarrow{ }^{*} U_{q}^{\sigma}
$$

$(X \otimes Y \otimes Z \rightarrow X Y Z)$. Then, letting $I^{ \pm}=\operatorname{ker}\left({ }^{M \#} \Psi_{q}\right) \mid \tilde{U}_{q}^{ \pm}$, we have

$$
\operatorname{ker}^{M \#} \Psi_{q}=I^{+} T \tilde{U}_{q}^{-}+\tilde{U}_{q}^{+} T^{\sharp} I^{-} \text {. }
$$

Moreover, for $\beta \in P_{+}$, we have $\left[{ }^{\sharp} I_{\beta}^{+}, F_{i}\right] \subset{ }^{\sharp} I_{\beta-\alpha_{i}}^{+} T$ and $\left[E_{i},{ }^{\sharp} I_{-\beta}^{-}\right] \subset T^{\sharp} I_{\alpha_{i}-\beta}^{-}$. Hence, by using the same argument as in the proof of Proposition 6.5.1, $\left\langle{ }^{\sharp} I_{\beta}^{+},\left(\widetilde{U}_{q}^{+}\right)_{\beta}\right\rangle$ $=\{0\}$ and $\left\langle\phi\left({ }^{\sharp} I_{-\beta}^{-}\right),\left(\tilde{U}_{q}^{+}\right)_{\beta}\right\rangle=\{0\}$. Hence ${ }^{\#} I^{ \pm} \subset I^{ \pm}$. Hence we have ker ${ }^{M \#} \Psi_{q}+$ $\sigma \operatorname{ker}^{M \#} \Psi_{q} \subset J^{+}+J^{-}$, as desired.

Q. E. D.

\section{§7. $q$-Analogues of the Isomorphisms}

7.1. Let $(\mathscr{E}, \Pi, p, \Gamma)$ be a lattice datum. Let $\mathscr{H}=\mathscr{E}^{*}$ (see 1.2). Let $U_{q}^{\sigma}=U_{q}^{\sigma}(\mathscr{E}$, $\Pi, p, \Gamma)$ and $U_{\mathbf{A}}^{\sigma}=U_{\mathbf{A}}^{\sigma}(\mathscr{E}, \Pi, p, \Gamma)$ be as in $\S 6$. Let $\mathbf{C}[[h]]$ be the C-algebra of formal power series in $h$. Let $\mathbf{S}_{\mathbf{A}}(\mathscr{H})$ be the symmetric $\mathbf{A}$-algebra of $\mathscr{H}$. Since $U_{\boldsymbol{A}}^{\sigma}$ $=\oplus_{\alpha \in P}\left(U_{\mathbf{A}}^{\sigma}\right)_{\alpha}$ is the $P$-graded algebra, we can consider an A-algebra $W_{\mathbf{A}}^{\sigma}=W_{\mathbf{A}}^{\sigma}(\mathscr{E}$, $\Pi, p, \Gamma)=U_{\mathbf{A}}^{\sigma} \otimes_{\mathbf{A}} \mathbf{S}_{\mathbf{A}}(\mathscr{H})$ such that

$$
\left(X \otimes \prod_{i} H_{\nu(i)}\right)\left(Y \otimes \prod_{j} H_{\mu(j)}\right)=X Y \otimes\left(\prod_{i}\left(H_{\nu(i)}+(\beta, \nu(i))\right)\right)\left(\prod_{J} H_{\mu(j)}\right)
$$

where $X \in U_{A}^{\sigma}, Y \in\left(U_{A}^{\sigma}\right)_{\beta}, \nu(i), \mu(j) \in \mathscr{E}$. For $X \in U_{A}^{\sigma}$ (resp. $H \in \mathscr{H}$ ), we also denote the element $X \otimes 1$ (resp. $1 \otimes H$ ) of $W_{\mathbf{A}}^{\sigma}$ by $X$ (resp. $H$ ). Moreover $W_{\mathbf{A}}^{\sigma}$ has a Hopf A-algebra structure such that $U_{\mathbf{A}}^{\sigma}$ is a Hopf subalgebra of $W_{\mathbf{A}}$ and that $\Delta(H)=H \otimes 1+1 \otimes H, S(H)=-H$ and $\varepsilon(H)=0$ for $H \in \mathscr{H}$. We view $\mathbb{A}$ as an $\mathbf{A}-$ subalgebra of $\mathbf{C}[[h]]$ by putting $q=\exp (h)$. Let $W_{\mathbf{A}}^{\sigma} \otimes \mathbf{C}[[h]]$ be the extension of the base algebra from $\mathbf{A}$ to $\mathbb{C}[[h]]$. Let $W_{h}^{\sigma}=W_{h}^{\sigma}(\mathscr{E}, \Pi, p, \Gamma)$ be the completion of $W_{\mathbf{A}}^{\sigma} \otimes \mathbf{C}[[h]]$ under the $h$-adic topology. Then $W_{h}^{\sigma}$ has a topological Hopf algebra structure induced from the Hopf algebra structure of $W_{\mathbf{A}}^{\sigma}$ (see $[\mathrm{Y} 1,1.2]$ for terminology). Let $J$ be an ideal of $W_{h}^{\sigma}$ generated by the elements $K_{\gamma}-\exp \left(h H_{\gamma}\right)$, $\left[K_{\gamma}\right]-\left[\exp \left(h H_{\gamma}\right)\right](\gamma \in \Gamma)$ where $\left[\exp \left(h H_{\gamma}\right)\right]$ is $\frac{\exp \left(h H_{\gamma}\right)-\exp \left(-h H_{\tau}\right)}{q-q^{-1}}$. Let $\bar{J}$ be the closure of $J$. Then $\bar{J}$ is a Hopf ideal of $W_{h}^{\sigma}$. We define a topological Hopf $\mathbb{C}[[h]]$ algebra $U_{h}^{\sigma}=U_{h}^{\sigma}(\mathscr{E}, \Pi, p, \Gamma)$ as the quotient Hopf algebra 


$$
U_{h}^{\sigma}=U_{h}^{\sigma}(\mathscr{E}, \Pi, p, \Gamma)=W_{h}^{\sigma} / \bar{J}
$$

For $\mathbf{C}[[h]]$-modules $V$ and $W$ which are completions of $V_{\mathbf{A}} \otimes_{\mathbf{A}} \mathbf{C}[[h]]$ and $W_{\mathbf{A}} \otimes_{\mathbf{A}}$ $\mathbf{C}[[h]]$, let $\boldsymbol{V} \hat{\otimes} W$ denote the completion of $\left(V_{\mathbf{A}} \otimes_{\mathbf{A}} W_{\mathbf{A}}\right) \otimes_{\mathbf{A}} \mathbf{C}[[h]]$. Let $U_{h}^{+}$(resp. $U_{h}^{-}$, resp. $\mathbf{S}_{h}(\mathscr{H})$ ) be the completion of $U_{\mathbf{A}}^{+} \otimes \mathbf{C}[[h]]$ (resp. $U_{\mathbf{A}}^{-} \otimes \mathbf{C}[[h]]$, resp. $\left.\mathbf{S}_{\mathbf{A}}(\mathscr{H}) \otimes \mathbf{C}[[h]]\right)$. Then we have a $\mathbf{C}[[h]]$-module isomorphism

$$
U_{h}^{+} \hat{\otimes} \mathbf{S}_{h}(\mathscr{H})^{\sigma} \hat{\otimes} U_{h}^{-} \rightarrow U_{h}^{\sigma}
$$

$(X \otimes Z \otimes Y \rightarrow X Z Y)$. In particular, the Hopf A-algebra $U_{A}^{\sigma}$ can be embedded into the topological Hopf $\mathbf{C}[[h]]$-algebra $U_{h}^{\sigma}$ where the elements $K_{\gamma},\left[K_{\gamma}\right], E_{i}, F_{i}$ of $U_{\mathrm{A}}^{\sigma}$ are identified with the elements $\exp \left(h H_{\gamma}\right),\left[\exp \left(h H_{\gamma}\right)\right], E_{i}, F_{i}$ of $U_{h}^{\sigma}$.

Remark 7.1.1. In $[\mathrm{Y} 1]$, we introduced a topological Hopf $\mathbf{C}[[h]]$-algebra $U_{h}^{\sigma}(\mathscr{E}, \Pi, p)$ for any datum $(\mathscr{E}, \Pi, p)$. If there is a lattice $\Gamma$ of $\mathscr{E}$ such that $(\mathscr{E}, \Pi$, $p, \Gamma)$ is a lattice datum, $U_{h}^{\sigma}(\mathscr{E}, \Pi, p)$ coincides with the above $U_{h}^{\sigma}(\mathscr{E}, \Pi, p, \Gamma)$. Let $U_{h}^{+}$be the topological $\mathbf{C}[[h]]$-subalgebra of $U_{h}^{\sigma}(\mathscr{E}, \Pi, p)$ genearated by the elements $E_{i}(0 \leq i \leq|\Pi|-1)$. We note that, if $(\mathscr{E}, \Pi, p) \in \operatorname{Data}\left(\mathrm{D}(2,1 ; x)^{(1)}\right), U_{h}^{+}$ can also be defined topologically by the generators $E_{i}(0 \leq i \leq 3)$ and the relations (QS4) (a) $(a=1,2,3)$ and

$$
\begin{array}{r}
(\mathrm{QS4})(7)(-1)^{p\left(\alpha_{i}\right) p\left(\alpha_{k}\right)}\left[\left(\alpha_{i}, a_{k}\right)\right] \llbracket \llbracket E_{i}, E_{j} \rrbracket, E_{k} \rrbracket \\
=(-1)^{p\left(\alpha_{i}\right) p\left(\alpha_{j}\right)}\left[\left(\alpha_{i}, \alpha_{j}\right)\right] \llbracket \llbracket E_{i}, E_{k} \rrbracket, E_{j} \rrbracket \\
\text { if }\left(\alpha_{i}, \alpha_{j}\right) \neq 0,\left(\alpha_{j}, \alpha_{k}\right) \neq 0,\left(\alpha_{k}, \alpha_{i}\right) \neq 0, \\
\left(\alpha_{i}, \alpha_{j}\right)+\left(\alpha_{j}, \alpha_{k}\right)+\left(\alpha_{k}, \alpha_{i}\right)=0 \\
\text { and } p\left(\alpha_{i}\right) p\left(\alpha_{j}\right)+p\left(\alpha_{j}\right) p\left(\alpha_{k}\right)+p\left(\alpha_{k}\right) p\left(\alpha_{i}\right)=\frac{1}{1}
\end{array}
$$

and

$(\mathrm{QS4})(8 x) \llbracket \llbracket \llbracket E_{j}, E_{i} \rrbracket, \llbracket E_{j}, E_{k} \rrbracket \rrbracket, \llbracket E_{j}, E_{l} \rrbracket \rrbracket=[x] \llbracket \llbracket \llbracket E_{j}, E_{i} \rrbracket, \llbracket E_{j}, E_{l} \rrbracket \rrbracket, \llbracket E_{j}, E_{k} \rrbracket \rrbracket$

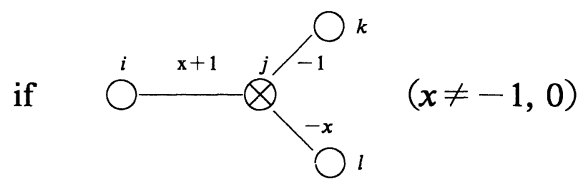

where $[y]=\frac{\exp (y h)-\exp (-y h)}{\exp (h)-\exp (-h)}$ for $y \in \mathbf{C}$.

Let $\mathbf{F}=\mathbf{C}((h))$ be the quotient field of $\mathbf{C}[[h]]$. For $\mathbf{F}$-algebras $V_{\mathbf{F}}$ and $W_{\mathbf{F}}$ such that $V_{\mathbf{F}}=V \otimes_{\mathbf{C}[h]]} \mathbf{F}$ and $W_{\mathbf{F}}=W \otimes_{\mathbf{C}[[h]]} \mathbf{F}$, let $V_{\mathbf{F}} \hat{\otimes} W_{\mathbf{F}}$ be $(V \hat{\otimes} W) \otimes \mathbf{F}$ if $V \hat{\otimes} W$ 
can be defined. Let $(\mathscr{E}, \Pi, p, \Gamma)$ be a lattice datum. It is clear that $U_{\mathrm{F}}^{o}=U_{\mathrm{F}}^{o}(\mathscr{E}, \Pi$, $p, \Gamma)=U_{h}^{\sigma}(\mathscr{E}, \Pi, p, \Gamma) \otimes_{\mathbf{C}[[h]]} \mathbf{F}$ is a topological Hopf $\mathbf{F}$-algebra. We have an F-linear isomorphism

$$
U_{\mathbf{F}}^{+} \hat{\otimes} \mathbf{S}_{\mathbf{F}}(\mathscr{H})^{\sigma} \hat{\otimes} U_{\mathbf{F}}^{-} \rightarrow U_{\mathbf{F}}^{\sigma}
$$

$(X \otimes Z \otimes Y \rightarrow X Z Y)$ where $U_{\mathbf{F}}^{ \pm}=U_{h}^{ \pm} \otimes \mathbf{F}$ and $\mathbf{S}_{\mathbf{F}}(\mathscr{H})^{\sigma}=\mathbf{S}_{h}(\mathscr{H})^{\sigma} \otimes \mathbb{F}$. Moreover the Hopf $\mathbf{C}(q)$-algebra $U_{q}^{\sigma}=U_{q}^{\sigma}(\mathscr{E}, \Pi, p, \Gamma)$ can be embedded into the topological Hopf F-algebra $U_{\mathrm{F}}^{\sigma}$ where the elements $q, K_{\gamma}, E_{i}, F_{i}$ of $U_{q}^{\sigma}$ are identified with the elements $\exp (h), \exp \left(h H_{\gamma}\right), E_{i}, F_{i}$ of $U_{\mathbf{F}}^{\sigma}$.

7.2. Let $\mathscr{U}$ be a topological Hopf F-algebra with comultiplication $\Delta$. Let $\Delta^{\prime}=$ $\varpi \circ \Delta$ where $\varpi(X \otimes Y)=Y \otimes X$. Let $\mathscr{Z}$ be a topological Hopf subalgebra of $\mathscr{U}$ such that there is an invertible element $R=\sum a_{i} \otimes b_{i}$ of $\mathscr{Z} \otimes \mathscr{Z}$ satisfying:

$$
\begin{gathered}
R \Delta(x) R^{-1}=\Delta^{\prime}(x) \quad \text { for any } \quad x \in \mathscr{Z}, \\
(\Delta \otimes I)(R)=R_{13} R_{23}, \quad(I \otimes \Delta)(R)=R_{13} R_{12}
\end{gathered}
$$

where $R_{12}=R \otimes I, R_{23}=I \otimes R$ and $R_{13}=\sum a_{i} \otimes I \otimes b_{i}$.

An R-triple $(\mathscr{U}, \mathscr{Z}, R)$ is a triple of $\mathscr{U}, \mathscr{Z}$ and $R$ with the above properties. The following lemma is easily verified:

Lemma 7.2.1. If $(\mathscr{U}, \mathscr{Z}, R)$ is an R-triple, then $\left(\mathscr{U}, \mathscr{Z}, \varpi\left(R^{-1}\right)\right)$ is also an R-triple.

The following proposition is well-known (see Drinfeld [D2]):

Proposition 7.2.2. Let $(\mathscr{U}, \mathscr{Z}, R)$ be an R-triple.

(i) $R$ satisfies:

$$
\begin{gathered}
R_{12} R_{13} R_{23}=R_{23} R_{13} R_{13}, \\
(S \otimes I)(R)=R^{-1}=\left(I \otimes S^{-1}\right)(R), \\
(\varepsilon \otimes I)(R)=1=(I \otimes \varepsilon)(R) .
\end{gathered}
$$

(ii) For $R=\sum a_{i} \otimes b_{i}$, the following equalities hold in $\mathscr{Z}$ :

$$
\begin{gathered}
\sum a_{i} S^{-2}\left(b_{i}\right)=\sum S\left(a_{i}\right) S^{-1}\left(b_{i}\right)=\sum S^{2}\left(a_{i}\right) b_{i}, \\
\sum a_{i} S\left(b_{i}\right)=\sum S^{-1}\left(a_{i}\right) b_{i} .
\end{gathered}
$$


Let $u_{4}, v_{4} \in \mathscr{Z}$ be the elements of (7.2.6), (7.2.7) respectively. Then $u_{4} v_{4}=1=$ $v_{4} u_{4}$.

The following proposition is also well-known:

Proposition 7.2.3. Let $(\mathscr{U}, \mathscr{Z}, R)$ be an R-triple. Then $\mathscr{U}$ has a topological Hopf F-algebra structure $\mathscr{U}^{(R)}=\left(\mathscr{U}, \Delta^{(R)}, S^{(R)}, \varepsilon\right)$ with comultiplication $\Delta^{(R)}$, antipode $S^{(R)}$, and counit $\varepsilon$ such that

$$
\Delta^{(R)}(x)=R \Delta(x) R^{-1}, S^{(R)}(x)=u_{4}^{-1} S(x) u_{4} \text { for } x \in \mathscr{U}
$$

This can be checked directly.

7.3. Keep the notation as above. We note that $U_{h}^{\sigma}$ can naturally be embedded into $U_{\mathrm{F}}^{\sigma}$. Put $t_{0}=\sum H_{\delta_{i}} \otimes H_{\delta_{i}} \in \mathscr{H} \otimes_{\mathbf{C}} \mathscr{H}$ where $\left\{\delta_{i}\right\}$ is a $\mathbf{C}$-basis of $\mathscr{H}$ such that $\left(\delta_{i}, \delta_{j}\right)$ $=\delta_{i j}$. Put

$$
\mathscr{C}=\frac{1}{2}\left(\sum_{c, d=0,1}(-1)^{c d} \sigma^{c} \otimes \sigma^{d}\right) \cdot \exp \left(-h t_{0}\right) \in U_{\mathrm{F}}^{\sigma} \hat{\otimes} U_{\mathbf{F}}^{\sigma} .
$$

Then $\left(U_{\mathbf{F}}^{\sigma}, \mathbf{S}_{\mathbf{F}}(\mathscr{H})^{\sigma}, \mathscr{C}\right)$ is an R-triple (see [Y1, Lemma 2.9.1]).

For $t \in \mathbf{C}[[h]]$ and $m, n>0$, we put $\{n\}_{t}=\frac{t^{n}-1}{t-1},\{n\}_{t} !=\{n\}_{t}\{n-1\}_{t} \cdots\{1\}_{t}$ and

$$
\left\{\begin{array}{l}
n \\
m
\end{array}\right\}_{t}=\left\{\begin{array}{cl}
\frac{\{n\}_{t} !}{\{m\}_{t} !\{n-m\}_{t} !} & \text { if } n \geq m \geq 0 \\
0 & \text { otherwise. }
\end{array}\right.
$$

Let $u \in h U_{\mathrm{F}}^{\sigma}$. Put $e(u, t)=\sum_{n=0}^{\infty} \frac{u^{n}}{\{n\}_{t} !}$. For $x \in \mathbf{C}[[h]]$ and $X, Y \in U_{\mathrm{F}}^{\sigma}$, define $\operatorname{ad}_{x}(X)(Y)=[X, Y]_{-, x} \in U_{F}^{\sigma}$ by $X Y-x Y X$. It is easily verified that $e\left(-u, t^{-1}\right)=$ $e(u, t)^{-1}$ and

$$
e(u, t) X e(u, t)^{-1}=\sum_{n=0}^{\infty} \frac{1}{\{n\}_{t} !} \operatorname{ad}_{t^{n-1}}(u) \operatorname{ad}_{t^{n-2}}(u) \cdots \operatorname{ad}_{1}(u)(X)
$$

for $X \in U_{\mathrm{F}}^{\sigma}$. Let $\left(\mathscr{E}, \Pi=\left\{\alpha_{0}, \ldots, \alpha_{n}\right\}, p, \Gamma\right)$ be a lattice datum. For a fixed $i, 0 \leq$ $i \leq n$, let $\mathscr{U}_{i}$ be a topological subalgebra of $U_{\mathrm{F}}^{\sigma}(\mathscr{E}, \Pi, p, \Gamma)$ generated by $E_{i}, F_{i}$ and $\mathbf{S}_{\mathbf{F}}(\mathscr{H})^{\sigma}$. Put

$$
R(i)=e\left(-\left(q-q^{-1}\right) E_{i} \otimes F_{i} \sigma^{p\left(\alpha_{i}\right)},(-1)^{p\left(\alpha_{i}\right)} q^{\left(\alpha_{i}, \alpha_{i}\right)}\right) \cdot \mathscr{C} \in \mathscr{U}_{i} \otimes \mathscr{U}_{i}
$$

Then $\left(U_{\mathrm{F}}^{\sigma}, \mathscr{U}_{i}, R(i)\right)$ is an R-triple (see [Y1, Lemma 2.9.1]). Let $\left(U_{\mathrm{F}}^{\sigma}\right)^{(i)}=\left(U_{\mathrm{F}}^{\sigma}\right.$, $\left.\Delta^{(i)}, S^{(i)}, \varepsilon\right)$ be the topological Hopf F-algebra defined by $\left(\left(U_{\mathrm{F}}^{g}\right)^{(R(i))}\right)^{\left(\mathscr{C}^{-1}\right)}$. Put 


$$
\hat{R}(i)=e\left(-\left(q-q^{-1}\right) \sigma^{p(\alpha)} K_{i}^{-1} E_{i} \otimes F_{i} K_{i},(-1)^{p\left(\alpha_{i}\right)} q^{\left(\alpha_{i}, \alpha_{i}\right)}\right) .
$$

Then we have $\hat{R}(i)=\mathscr{C}^{-1} R(i)$. Hence

$$
\Delta^{(i)}(X)=\hat{R}(i) \Delta(X) \hat{R}(i)^{-1} \quad\left(X \in U_{\mathrm{F}}^{\sigma}\right) .
$$

Proposition 7.3.1. Keep the notation as above. Put $\alpha=\alpha_{i}, \beta=\alpha_{j} \in \Pi(i \neq j)$, and put $E_{\alpha}=E_{i}, E_{\beta}=E_{j}, F_{\alpha}=F_{i}, F_{\beta}=F_{j} . \quad$ Put $E_{\beta+s \alpha}^{\vee}=\llbracket \ldots \llbracket \llbracket E_{\beta}, E_{\alpha} \rrbracket, E_{\alpha} \rrbracket \ldots E_{\alpha} \rrbracket\left(E_{\alpha}\right.$ appears $s$-times), $F_{\beta+s \alpha}^{\vee}=\llbracket \ldots \llbracket \llbracket F_{\beta}, F_{\alpha} \rrbracket, F_{\alpha} \rrbracket \ldots F_{\alpha} \rrbracket$ ( $F_{\alpha}$ appears $s$-times).

(i)

$$
\begin{gathered}
\Delta^{(i)}\left(E_{i} K_{i}^{-1}\right)=E_{i} K_{i}^{-1} \otimes K_{i}+\sigma^{p\left(\alpha_{i}\right)} \otimes E_{i} K_{i}^{-1}, \\
\Delta^{(i)}\left(K_{i} F_{i}\right)=K_{i} F_{i} \otimes 1+\sigma^{p\left(\alpha_{i}\right)} K_{i}^{-1} \otimes K_{i} F_{i},
\end{gathered}
$$

(ii) Assume $(\alpha, \alpha) \neq 0$. For $\beta \in \Pi$, assume $r=r_{\alpha, \beta}=-\frac{2(\alpha, \beta)}{(\alpha, \alpha)} \in \mathbf{Z}_{+}$and $p(\alpha) \cdot r$ is even. Then

$$
\begin{aligned}
& \Delta^{(i)}\left(E_{\beta+r \alpha}^{\vee}\right)=E_{\beta+r \alpha}^{\vee} \otimes 1+K_{\beta+r \alpha} \sigma^{p(\beta+r \alpha)} \otimes E_{\beta+r \alpha}^{\vee}, \\
& \Delta^{(i)}\left(F_{\beta+r \alpha}^{\vee}\right)=F_{\beta+r \alpha}^{\vee} \otimes K_{\beta+r \alpha}^{-1}+\sigma^{p(\beta+r \alpha)} \otimes F_{\beta+r \alpha}^{\vee} .
\end{aligned}
$$

(iii) Assume $(\alpha, \alpha)=0$ and $(\alpha, \beta) \neq 0$. Then

$$
\begin{aligned}
& \Delta^{(i)}\left(E_{\beta+\alpha}^{\vee}\right)=E_{\beta+\alpha}^{\vee} \otimes 1+K_{\beta+\alpha} \sigma^{p(\beta+\alpha)} \otimes E_{\beta+\alpha}^{\vee}, \\
& \Delta^{(i)}\left(F_{\beta+\alpha}^{\vee}\right)=F_{\beta+\alpha}^{\vee} \otimes K_{\beta+\alpha}^{-1}+\sigma^{p(\beta+\alpha)} \otimes F_{\beta+\alpha}^{\vee} .
\end{aligned}
$$

(iv) $\Delta^{(i)}(H)=H \otimes 1+1 \otimes H$ for $H \in \mathscr{H}$, and $\Delta^{(i)}(\sigma)=\sigma \otimes \sigma$.

Proof. For $\mu, \nu \in P$, let $t_{\mu}=(-1)^{p(\mu)} q^{(\mu, \mu)}, t_{\mu, \nu}=(-1)^{p(\mu) p(\nu)} q^{(\mu, \nu)}$ and

$$
\{k ; \nu\}_{\mu}=\frac{q^{-(\mu, \nu)}\left(1-t_{\mu}^{-k}\right)\left(1-t_{\mu, \nu}^{2} t_{\mu}^{k-1}\right)}{\left(q-q^{-1}\right)\left(1-t_{\mu}^{-1}\right)} .
$$

We first note the following formulas

$$
\begin{aligned}
& {\left[E_{\alpha}, F_{\beta+k \alpha}^{\vee}\right]=-t_{\alpha, \beta}^{-1} q^{-(k-1)(\alpha, \alpha)}\{k ; \beta\}_{\alpha} F_{\beta+(k-1) \alpha}^{\vee} K_{\alpha}} \\
& {\left[E_{\beta+k \alpha}^{\vee}, F_{\alpha}\right]=(-1)^{(k-1) p(\alpha)}\{k ; \beta\}_{\alpha} K_{\alpha}^{-1} E_{\beta+(k-1) \alpha}^{\vee} .}
\end{aligned}
$$

By direct calculation, we have 


$$
\begin{aligned}
\Delta\left(E_{\beta+r \alpha}^{\vee}\right) & =E_{\beta+r \alpha}^{\vee} \otimes 1 \\
& +\sum_{s=0}^{r}\left\{\begin{array}{l}
r \\
s
\end{array}\right\}_{t_{\alpha}} t_{\alpha, \beta}^{r-s} \prod_{k=1}^{r-s}\left(1-t_{\alpha}^{k}\right) E_{\alpha}^{r-s} K_{\beta+s \alpha} \sigma^{p(\beta+s \alpha)} \otimes E_{\beta+s \alpha}^{\vee} .
\end{aligned}
$$

Put $X=-\sigma^{p(\alpha)} K_{i}^{-1} E_{i} \otimes F_{i} K_{i}$. Then, by (7.3.1), we have

$$
\begin{aligned}
& {\left[-X, E_{\alpha}^{s} K_{\beta+(r-s) \alpha} \sigma^{p(\beta+(r-s) \alpha)} \otimes E_{\beta+(r-s) \alpha}^{\vee}\right]_{-, t^{-s}}} \\
& \quad\left\{\begin{array}{l}
-\frac{t_{\alpha, \beta}}{q-q^{-1}} t_{\alpha}^{-s} \frac{\left(t_{\alpha}^{r-s}-1\right)\left(t_{\alpha}^{s+1}-1\right)}{t_{\alpha}-1} E_{\alpha}^{s+1} K_{\beta+(r-s-1) \alpha} \sigma^{p(\beta+(r-s-1) \alpha)} \otimes E_{\beta+(r-s-1) \alpha}^{\vee} \\
\quad \text { if } r \geq s, \\
0 \quad \text { if } r<s .
\end{array}\right.
\end{aligned}
$$

Hence

$$
\begin{aligned}
\operatorname{ad}_{t_{\alpha}^{-}(s-1)} & (X) \operatorname{ad}_{t_{\alpha}^{-}(s-2)}(X) \cdots \operatorname{ad}_{1}(X)\left(K_{\beta+r \alpha} \sigma^{p(\beta+r \alpha)} \otimes E K_{\beta+r \alpha}^{\vee}\right) \\
= & \left\{\begin{array}{c}
\left(\frac{t_{\alpha, \beta}}{q-q^{-1}}\right)^{s} t_{\alpha}^{-\frac{s(s-1)}{2}}\left(t_{\alpha}-1\right)^{s}\left\{\begin{array}{r}
r \\
s
\end{array}\right\}_{t_{\alpha}} E_{\alpha}^{s} K_{\beta+(r-s) \alpha} \sigma^{p(\beta+(r-s) \alpha)} \otimes E_{\beta+(r-s) \alpha}^{\vee} \\
\text { if } r \geq s, \\
0 \quad \text { if } r<s .
\end{array}\right.
\end{aligned}
$$

Hence, by (7.3.1),

$$
\begin{aligned}
\hat{R}(i)^{-1}\left(K_{\beta+r \alpha} \sigma^{p(\beta+r \alpha)} \otimes E_{\beta+r \alpha}^{\vee}\right) \hat{R}(i) \\
\quad=\sum_{s=0}^{r}\left(-t_{\alpha}\right)^{s}(t-1)^{s} \frac{\{r\}_{t_{\alpha}} !}{\{r-s\}_{t_{\alpha}} !} E_{\alpha}^{s} K_{\beta+(r-s) \alpha} \sigma^{p(\beta+(r-s) \alpha)} \otimes E_{\beta+(r-s) \alpha}^{\vee} .
\end{aligned}
$$

On the other hand, we can easily show $\hat{R}(i)^{-1}\left(E_{\beta+r \alpha}^{\vee} \otimes 1\right) \hat{R}(i)=E_{\beta+r \alpha}^{\vee} \otimes 1$. Then we get:

$$
\hat{R}(i)^{-1}\left(\mathrm{E}_{\beta+r \alpha}^{\vee} \otimes 1+K_{\beta+r \alpha} \sigma^{p(\beta+r \alpha)} \otimes E_{\beta+r \alpha}^{\vee}\right) \hat{R}(i)=\Delta\left(E_{\beta+r \alpha}^{\vee}\right) .
$$

Thus we have proved the first statement in (ii). In other cases, the corresponding calculation can be carried out similarly and, usually, much more easily.

Q. E. D.

7.4. Keep the notation as above. For $\nu, \mu \in P$, put 


$$
z(\nu, \mu, k)=(-1)^{k} t_{\nu, \mu}^{-k} q^{-\frac{k(k-1)}{2}(\nu, \nu)}\{k ; \mu\}_{\nu} !
$$

where $\{k ; \mu\}_{\nu} !=\{k ; \mu\}_{\nu}\{k-1 ; \mu\}_{\nu} \cdots\{1 ; \mu\}_{\nu}$. Put $\alpha=\alpha_{i}, \beta=\alpha_{j} \in \Pi(i \neq j)$. By using (7.3.2-3), and by induction, we have:

$$
\begin{aligned}
& {\left[E_{\beta+k \alpha}^{\vee}, F_{\beta+(k-1) \alpha}^{\vee}\right]} \\
& \quad=(-1)^{k(1+p(\alpha) p(\beta))} q^{-\frac{(k-1)(k-2)}{2}(\alpha, \alpha)} q^{(-k+1)(\alpha, \beta)}\{k ; \beta\}_{\alpha} ! E_{\alpha} K_{\beta+(k-1) \alpha}, \\
& \quad\left[E_{\beta+(k-1) \alpha}^{\vee}, F_{\beta+k \alpha}^{\vee}\right] \\
& \quad=(-1)^{(k-1)(1+p(\alpha)+p(\alpha) p(\beta))} q^{-\frac{k(k-1)}{2}(\alpha, \alpha)} q^{-k(\alpha, \beta)}\{k ; \beta\}_{\alpha} ! K_{\beta+(k-1) \alpha}^{-1} F_{\alpha}
\end{aligned}
$$

and

$$
\left[E_{\beta+k \alpha}^{\vee}, F_{\beta+k \alpha}^{\vee}\right]=z(\alpha, \beta, k) \frac{K_{\beta+k \alpha}-K_{\beta+k \alpha}^{-1}}{q-q^{-1}} .
$$

Let $\left(\mathscr{E}, \Pi=\left\{\alpha_{0}, \ldots, \alpha_{n}\right\}, p, \Gamma\right)$ be a lattice datum. Fix $i, 0 \leq i \leq n$. We say that $\left\{x_{j}\right.$, $\left.y_{j} \in \mathbb{C}(q) \backslash\{0\} \mid 0 \leq j \leq n\right\}$ is a tuning of $(\mathscr{E}, \Pi, p, \Gamma)$ with respect to the simple root $\alpha_{i}$ if

$$
\frac{1}{x_{j} y_{j}}=\left\{\begin{array}{cl}
(-1)^{p\left(\alpha_{i}\right)} & \text { if } j=i, \\
z\left(\alpha_{i}, \alpha_{j},-\frac{2\left(\alpha_{i}, \alpha_{j}\right)}{\left(\alpha_{i}, \alpha_{i}\right)}\right) & \text { if }\left(\alpha_{i}, \alpha_{i}\right) \neq 0 \text { and } j \neq i, \\
\mathrm{z}\left(\alpha_{\mathrm{i}}, \alpha_{j}, 1\right) & \text { if }\left(\alpha_{i}, \alpha_{i}\right)=0 \text { and } j \neq i .
\end{array}\right.
$$

Put $E_{\beta+k \alpha}^{\wedge}=\llbracket E_{\alpha}, \ldots \llbracket E_{\alpha}, \llbracket E_{\alpha}, E_{\beta} \rrbracket \rrbracket \ldots\left(E_{\alpha}\right.$ appears $k$-times $)$ and $\boldsymbol{F}_{\beta+k \alpha}^{\wedge}=\llbracket F_{\alpha}, \ldots$ $\llbracket F_{\alpha}, \llbracket F_{\alpha}, F_{\beta} \rrbracket \rrbracket \ldots\left(F_{\alpha}\right.$ appears $k$-times $)$.

Proposition 7.4.1 (see also $[\mathrm{KT}])$. Let $\left(\mathscr{E}, \Pi=\left\{\alpha_{0}, \ldots, \alpha_{n}\right\}, p, \Gamma\right)$ and $\left(\mathscr{E}^{\prime}\right.$, $\left.\Pi^{\prime}=\left\{\alpha_{0}^{\prime}, \ldots, \alpha_{n}^{\prime}\right\}, p^{\prime}, \Gamma^{\prime}\right)$ be lattice data. Fix $i, 0 \leq i \leq n$. Assume that there is a reflection (resp. super-reflection) $f: \mathscr{E} \rightarrow \mathscr{E}^{\prime}$ with respect to the simple root $\alpha_{i}$ if $\left(\alpha_{i}, \alpha_{i}\right)$ $\neq 0\left(\right.$ resp. $\left.\left(\alpha_{i}, \alpha_{i}\right)=0\right)$. Assume $f(\Gamma)=\Gamma^{\prime}$. Let $\left\{x_{j}^{\prime}, y_{j}^{\prime} \in \mathbf{C}(q) \backslash\{0\} \mid 0 \leq j \leq n\right\}$ be a tuning of $\left(\mathscr{E}^{\prime}, \Pi^{\prime}, p^{\prime}, \Gamma^{\prime}\right)$ with respect to the simple root $\alpha_{i}^{\prime}$. Then there is a $\mathbf{C}(q)$ algebra homomorphism $\mathscr{L}_{i}^{\wedge}: U_{q}^{\sigma}(\mathscr{E}, \Pi, p, \Gamma) \rightarrow U_{q}^{\sigma}\left(\mathscr{E}^{\prime}, \Pi^{\prime}, p^{\prime}, \Gamma^{\prime}\right)$ such that $\mathscr{L}_{i}^{\wedge}(\sigma)$ $=\sigma, \mathscr{L}_{i}^{\wedge}\left(K_{\gamma}\right)=K_{f(\gamma)}, \mathscr{L}_{i}^{\wedge}\left(E_{i}\right)=x_{i}^{\prime} K_{i}^{-1} F_{i}, \mathscr{L}_{i}^{\wedge}\left(E_{j}\right)=x_{j}^{\prime} E_{f\left(\alpha_{j}\right)}^{\wedge}(j \neq i), \mathscr{L}_{i}^{\wedge}\left(F_{i}\right)=y_{i}^{\prime} E_{i} K_{i}$ and $\mathscr{L}_{i}^{\wedge}\left(F_{j}\right)=y_{j}^{\prime} F_{f\left(\alpha_{j}\right)}^{\wedge}(j \neq i)$.

Proof. Let $\left\{x_{j}, y_{j} \in \mathbf{C}(q) \backslash\{0\} \mid 0 \leq j \leq n\right\}$ be a tuning of $(\mathscr{E}, \Pi, p, \Gamma)$ with respect to the simple root $\alpha_{i}$. Let $g=f^{-1}$. By Proposition 7.3.1, (7.3.2-3), (7.4.1) and Proposition 6.12.1, we see that there is a homomorphism ' $\mathscr{L}_{i}^{\wedge}: U_{q}^{\sigma}(\mathscr{E}, \Pi, p, \Gamma) \rightarrow$ $U_{q}^{\sigma}\left(\mathscr{E}^{\prime}, \Pi^{\prime}, p^{\prime}, \Gamma^{\prime}\right)$ such that ${ }^{\prime} \mathscr{L}_{i}^{\wedge}(\sigma)=\sigma,^{\prime} \mathscr{L}_{i}^{\wedge}\left(K_{g\left(\gamma^{\prime}\right)}\right)=K_{\gamma^{\prime}}\left(\gamma^{\prime} \in \Gamma^{\prime}\right),{ }^{\prime} \mathscr{L}_{i}^{\wedge}\left(y_{i} E_{i} K_{i}^{-1}\right)$ 
$=F_{i}, \mathscr{L}_{i}^{\wedge}\left(x_{j} E_{g\left(\alpha_{j}^{\prime}\right)}^{\vee}\right)=E_{j}(j \neq i),{ }^{\prime} \mathscr{L}_{i}^{\wedge}\left(x_{i} K_{i} F_{i}\right)=E_{i}$ and ${ }^{\prime} \mathscr{L}_{i}^{\wedge}\left(y_{j} F_{g\left(\alpha_{j}^{\prime}\right)}^{\vee}\right)=F_{j}(j \neq i)$. By using (7.3.2-3), we see that ' $\mathscr{L}_{i}^{\wedge}$ with a suitable $\left\{x_{j}, y_{j}\right\}$ is the desired map.

Q. E. D.

By Lemma 6.3.1, we see that there is an anti-automorphism $r: U_{\mathrm{F}}^{\sigma} \rightarrow U_{\mathrm{F}}^{\sigma}$ such that $r(\sigma)=\sigma, r(H)=-H(H \in \mathscr{H}), r\left(E_{i}\right)=E_{i}$ and $r\left(F_{i}\right)=(-1)^{p\left(\alpha_{i}\right)} F_{i}$.

Proposition 7.4.2. Keep the hypothesis of Proposition 7.4.1. Let $\left\{x_{j}, y_{j} \in \mathbf{C}(q) \backslash\right.$ $\{0\} \mid 0 \leq j \leq n\}$ be a tuning of $(\mathscr{E}, \Pi, p, \Gamma)$ with respect to the simple root $\alpha_{i}$. Then there is a $\mathbf{C}(q)$-algebra homomorphism $\mathscr{L}_{i}^{\vee}: U_{q}^{\sigma}\left(\mathscr{E}^{\prime}, \Pi^{\prime}, p^{\prime}, \Gamma^{\prime}\right) \rightarrow U_{q}^{\sigma}(\mathscr{E}, \Pi, p, \Gamma)$ such that $\mathscr{L}_{i}^{\vee}(\sigma)=\sigma, \mathscr{L}_{i}^{\vee}\left(K_{\gamma^{\prime}}\right)=K_{f^{-1}\left(\gamma^{\prime}\right)}, \mathscr{L}_{i}^{\vee}\left(E_{i}\right)=x_{i} F_{i} K_{i}, \mathscr{L}_{i}^{\vee}\left(E_{j}\right)=x_{j} E_{f}^{\vee}\left(\alpha_{j}\right)(j \neq i)$, $\mathscr{L}_{i}^{\vee}\left(F_{i}\right)=y_{i} K_{i}^{-1} E_{i}$ and $\mathscr{L}_{i}^{\vee}\left(F_{j}\right)=y_{j} F_{f}^{\vee}{ }_{\left(\alpha_{j}\right)}(j \neq i)$. Moreover, for some $\left\{x_{j}, y_{j}\right\}, \mathscr{L}_{i}^{\vee}$ is the inverse map of $\mathscr{L}_{i}^{\wedge}$.

Proof. Define $\mathscr{L}_{i}^{\vee}$ as $r \circ \mathscr{L}_{i}^{\wedge} \circ$.

Q. E. D.

§. On Defining Relations of $U_{q}\left(\left(\mathbf{A}(m, m)^{(1)}\right)^{\mathscr{H}}\right)$ and the Drinfeld Generators

In $\S 8$, we follow Beck's argument $[\mathrm{B}]$.

8.1. For $N \geq 3$, let $\widetilde{\mathrm{AA}}(N)$ be the set of data $(\mathscr{E}, \Pi, p, \Gamma)$ such that the Dynkin diagram of $(\mathscr{E}, \Pi, p)$ is (AA) and $|\Pi|=N$ (see 1.7). Note that $(\mathscr{E}, \Pi, p, \Gamma)$ depends only on $(\mathscr{E}, \Pi, p)$ (see 6.7).

For a fixed $(\mathscr{E}, \Pi, p, \Gamma) \in \widetilde{\mathrm{AA}}(N)$ and a fixed $i, 0 \leq i \leq N-1$, we define $\left({ }^{t_{i}} \mathscr{E}\right.$, $\left.{ }^{t_{i}} \Pi,{ }^{t_{i}} p,{ }^{t_{i}} \Gamma\right) \in \widetilde{\mathrm{AA}}(N)$ by (a)-(b) below:

(a) Let $\left\{\bar{\varepsilon}_{1}, \ldots, \bar{\varepsilon}_{N}, \delta, \Lambda_{0}\right\}$ be the fundamental elements of $(\mathscr{E}, \Pi, p, \Gamma)$, and $\bar{d}_{i}=\left(\bar{\varepsilon}_{i}, \bar{\varepsilon}_{i}\right)= \pm 1$. Let $\left\{\bar{\varepsilon}_{1}^{\prime}, \ldots, \bar{\varepsilon}_{N}^{\prime}, \delta, \Lambda_{0}\right\}$ be the fundamental elements of $\left({ }^{t_{i}} \mathscr{E},{ }^{t_{i}} \Pi\right.$, $\left.{ }^{t_{i}} p\right)$, and $\bar{d}_{i}^{\prime}=\left(\bar{\varepsilon}_{i}^{\prime}, \bar{\varepsilon}_{i}^{\prime}\right)= \pm 1$.

(b ) Let $\left({ }^{t_{i}} \mathscr{E},{ }^{t_{i}} \Pi,{ }^{t_{i}} p,{ }^{t_{i}} \Gamma\right) \in \widetilde{\mathrm{AA}}(N)$ be such that $\bar{d}_{j+i}^{\prime}=\bar{d}_{j}$.

Let $\Pi=\left\{\alpha_{j}(0 \leq j \leq N-1)\right\}$ (resp. $\left.{ }^{t_{i}} \Pi=\left\{\alpha_{j}^{\prime}(0 \leq j \leq N-1)\right\}\right)$ be the set of the simple roots of $(\mathscr{E}, \Pi, p)$ (resp. $\left({ }^{t_{i}} \mathscr{E},{ }^{t_{i}} \Pi,{ }^{t_{i}} p\right)$ ) where the numbering of the simple roots is the same as in 1.4. Let $P$ (resp. ${ }^{{ }_{i}} P$ ) be the root lattices of $(\mathscr{E}, \Pi, p)$ and (resp. $\left({ }^{t_{i}} \mathscr{E}\right.$, $\left.{ }^{t_{i}} \Pi,{ }^{t_{i}} p\right)$ ). For a fixed $i, 0 \leq i \leq N-1$, define a parity preserving lattice isometry $t_{i}$ : $P \rightarrow{ }^{t_{i}} P$ by $t_{i}\left(\alpha_{j}\right)=\alpha_{j+i}^{\prime}$.

For each $(\mathscr{E}, \Pi, p, \Gamma) \in \widetilde{\mathrm{AA}}(N)$ and each $0 \leq i \leq N-1$, we fix a reflection or a super-reflection $s_{i}: \mathscr{E} \rightarrow{ }^{s_{i}} \mathscr{E}$ given in Proposition 2.4.3. Let $\left({ }^{s_{i}} \mathscr{E},{ }^{s_{i}} \Pi,{ }^{s_{i}} p,{ }^{s_{i}} \Gamma\right) \in \widetilde{\mathrm{AA}}(N)$ be defined for $\left({ }^{s_{i}} \mathscr{E},{ }^{s_{i}} \Pi,{ }_{i} p\right)$ (see 6.7). Let ${ }^{s_{i}} P$ be the root lattice of $\left({ }^{s_{i}} \mathscr{E},{ }^{s_{i}} \Pi,{ }^{s_{i}} p\right)$. By abuse of notation, we denote the parity preserving lattice isometry $s_{i \mid P}: P \rightarrow{ }^{s_{i}} P$ by $s_{i}$. 
We denote by $e: P \rightarrow P$ the identity map $\operatorname{id}_{P}$. Then we have

$$
\begin{gathered}
\left(s_{i}\right)^{2}=e, \\
s_{i} s_{j} s_{i}=s_{j} s_{i} s_{j},(j-i= \pm 1(\bmod N)), s_{i} s_{j}=s_{j} s_{i},(j-i \neq 0, \pm 1(\bmod N))
\end{gathered}
$$

and

$$
\left(t_{i}\right)^{N}=e, t_{i} t_{j}=t_{i+j}, t_{i} s_{j}\left(t_{i}\right)^{-1}=s_{i+j}
$$

For a composition $w=c_{i(1)} \cdots c_{i(r)}$ of $c_{i(k)}\left(=s_{i(k)}\right.$ or $\left.t_{i(k)}\right)$, define $\left({ }^{w} \mathscr{E},{ }^{w} \Pi,{ }^{w} p,{ }^{w} \Gamma\right)$ $\in \widetilde{\mathrm{AA}}(N)$ by

$$
\left.\left({ }^{w} \mathscr{E},{ }^{w} \Pi,{ }^{w} p,{ }^{w} \Gamma\right)={ }^{c_{i(1)}}\left(\ldots{ }^{c_{i(r-1)}}\left({ }^{c_{i}(r)}(\mathscr{E}, \Pi, p, \Gamma)\right)\right) \ldots\right)
$$

where ${ }^{c_{j}}(\mathscr{E}, \Pi, p, \Gamma)$ denotes $\left({ }^{c_{j}} \mathscr{E},{ }^{c_{j}} \Pi,{ }^{c_{j}} p,{ }^{c_{j}} \Gamma\right)$. We note that $\left({ }^{w} \mathscr{E},{ }^{w} \Pi,{ }^{w} p,{ }^{w} \Gamma\right)$ does not depend on the expression of $w$ as a product of $c_{j}$ 's. Let ${ }^{w} P$ be the root lattice of $\left({ }^{w} \mathscr{E},{ }^{w} \Pi,{ }^{w} p,{ }^{w} \Gamma\right)$. Then $w: P \rightarrow{ }^{w} P$ is a parity preserving lattice isometry. For $1 \leq i$ $\leq N-1$, we define the parity preserving lattice isometry $\omega_{i}: P \rightarrow P$, by

$$
\omega_{i}=t_{i} u_{1}^{(i)} u_{2}^{(i)} \cdots u_{N-i}^{(i)}
$$

where $u_{r}^{(i)}=s_{N-r-i+1} \cdots s_{N-r-1} s_{N-r}$. Then we have $\omega_{i}\left(\alpha_{j}\right)=\alpha_{j}-\delta_{i j} \delta(1 \leq i, j \leq N-1)$ and $\omega_{i}(\delta)=\delta$.

Let $\overline{\mathscr{W}}$ be the group defined with the generators $\bar{s}_{i}, \bar{t}_{i}(0 \leq i \leq N-1)$ and the relations same as in (8.1.1-3) with the unit element $\bar{e}$ of $\overline{\mathscr{W}}, \bar{s}_{i}$ and $t_{i}$ in place of $e$, $s_{i}$ and $t_{i}$. The group $\overline{\mathscr{W}}$ is well-known (see $[\mathrm{IM}],[\mathrm{L} 2]$ ) and it is called the extended affine Weyl group. For a composition $w=c_{i(1)} \cdots c_{i(r)}$ of $c_{i(k)}\left(=s_{i(k)}, t_{i(k)}\right)$, we denote by $\bar{w} \in \bar{W}$ the composition $\bar{w}=\bar{c}_{i(1)} \cdots \bar{c}_{i(r)}$ of $\bar{c}_{i(k)}\left(=\bar{s}_{i(k)}, \bar{t}_{i(k)}\right)$. We note that $\bar{w}$ does not depend on the expression of $w$ as a product of $c_{j}^{\prime}$ 's. When $\bar{w} \in \overline{\mathscr{W}}$ is written as $\bar{t}_{k} \bar{s}_{i(1)} \cdots \bar{s}_{i(a)}(a$ minimal), we call the expression reduced, and we also call the corresponding expression $t_{k} s_{i(1)} \cdots s_{i(a)}$ reduced. Note that the expression in (8.1.4) is reduced.

Let $\mathscr{Q}$ be the subgroup of $\overline{\mathscr{W}}$ generated by the elements $\bar{\omega}_{1}, \ldots, \bar{\omega}_{N-1}$. Then $\mathscr{Q}$ is a free abelian group (see $[\mathrm{IM}]$ ) with the generators $\bar{\omega}_{1}, \ldots, \bar{\omega}_{N-1}$. Let $\mathscr{W}_{0}$ be the subgroup of $\overline{\mathscr{W}}$ generated by the elements $\bar{s}_{1}, \ldots, \bar{s}_{N-1}$. Then $\overline{\mathscr{W}}$ is a semidirect product $\mathscr{W}_{0} \mathscr{Q}$. Let $\bar{\gamma}_{1}=\bar{\omega}_{1}^{2} \bar{\omega}_{2}^{-1}, \bar{\gamma}_{i}=\bar{\omega}_{i-1}^{-1} \bar{\omega}_{i}^{2} \bar{\omega}_{i+1}^{-1} \quad(2 \leq i \leq N-1)$ and $\bar{\gamma}_{N-1}=$ $\bar{\omega}_{N-2}^{-1} \bar{\omega}_{N-1}^{2}$. Then, for $1 \leq i, j \leq N-1$, we have $\bar{s}_{i} \bar{\omega}_{j} \bar{s}_{i}=\bar{\omega}_{j} \bar{\gamma}_{i}{ }^{-\delta_{i j}}$.

8.2. Let $\left(\mathscr{E}, \Pi=\left\{\alpha_{0}, \ldots, \alpha_{n}\right\}, p, \Gamma\right) \in \widetilde{\mathrm{AA}}(N)$. Let $\mathscr{D} U_{q}=\mathscr{D} U_{q}(\mathscr{E}, \Pi, p, \Gamma)$ be the $\mathrm{C}(q)$-subalgebra of $U_{\mathrm{F}}^{\sigma}$ generated by the elements $K_{\alpha}=\exp \left(h H_{\alpha}\right)(\alpha \in P), E_{i}, F_{i}$ $(0 \leq i \leq N-1)$ and $K_{\delta}^{ \pm \frac{1}{2}}=\exp \left( \pm \frac{1}{2} h H_{\delta}\right)$. Note 


$$
\left(\oplus_{m \in \mathbf{Z}} \mathbf{C}(q) K_{\delta}^{m \frac{k}{2}}\right) U_{q}=\left(\oplus_{\gamma \in \Gamma} \mathbf{C}(q) K_{\gamma}\right) \mathscr{D} U_{q}
$$

as $\mathbf{C}(q)$-subspaces of $U_{\mathrm{F}}^{\sigma}$. By Propositions 7.4.1-2 and (8.2.1), we have

Proposition 8.2.1. Let $\left(\mathscr{E}, \Pi=\left\{\alpha_{0}, \ldots, \alpha_{N-1}\right\}, p, \Gamma\right) \in \widetilde{\mathrm{AA}}(N)$. Fix $i, 1 \leq i \leq$ $N$. Let $\bar{\varepsilon}_{1}, \ldots, \bar{\varepsilon}_{N}$ be the fundamental elements of $(\mathscr{E}, \Pi, p, \Gamma)$, and let $\bar{d}_{i}=\left(\bar{\varepsilon}_{i}, \bar{\varepsilon}_{i}\right)$. Let $\bar{\varepsilon}_{1}^{\prime}, \ldots, \bar{\varepsilon}_{N}^{\prime}$ be the fundamental elements of $\left({ }^{s_{i}} \mathscr{E},{ }^{s} \Pi,{ }^{s_{i}} p,{ }^{s_{i}} \Gamma\right)$, and let $\bar{d}_{i}^{\prime}=\left(\bar{\varepsilon}_{i}^{\prime}, \bar{\varepsilon}_{i}\right)$ and $p^{\prime}={ }^{s_{i}} p$. Let ${ }^{s_{i}} \Pi=\left(\alpha_{0}^{\prime}, \ldots, \alpha_{N-1}^{\prime}\right\}$ be the simple roots of $\left({ }^{s_{i}} \mathscr{E},{ }^{s_{i}} \Pi,{ }^{s_{i}} p,{ }^{s_{i}} \Gamma\right)$. Then there exists an isomorphisms $T_{i}: \mathscr{D} U_{q}(\mathscr{E}, \Pi, p, \Gamma) \rightarrow \mathscr{D} U_{q}\left({ }^{s} \mathscr{E}^{s},{ }^{s_{i}} \Pi,{ }^{s_{i}} p,{ }^{s_{i}} \Gamma\right)$ such that

$$
\begin{aligned}
& T_{i}\left(K_{\alpha}\right)=K_{s_{i}(\alpha)}(\alpha \in P), T_{i}\left(K_{\delta}^{+\frac{1}{2}}\right)=K_{\delta}^{+\frac{1}{2}}, \\
& T_{i}\left(E_{i}\right)=-\bar{d}_{i+1}^{\prime} F_{i} K_{i}, T_{i}\left(F_{i}\right)=-\bar{d}_{i}^{\prime} K_{i}^{-1} E_{i}, \\
& T_{i}\left(E_{i-1}\right)=q^{-d_{i}^{\prime}} \bar{d}_{i}^{\prime} \llbracket E_{i-1}, E_{i} \rrbracket, T_{i}\left(F_{i-1}\right)=-(-1)^{p^{\prime}\left(\alpha_{i}^{\prime}\right) p^{\prime}\left(\alpha_{i-1}^{\prime}\right)} \llbracket F_{i-1}, F_{i} \rrbracket, \\
& T_{i}\left(E_{i+1}\right)=q^{-d_{i+1}^{\prime}}(-1)^{p^{\prime}\left(\alpha_{i}^{\prime}\right) p^{\prime}\left(\alpha_{i+1}^{\prime}\right)} \bar{d}_{i+1}^{\prime} \llbracket E_{i+1}, E_{i} \rrbracket, T_{i}\left(F_{i+1}\right)=-\llbracket F_{i+1}, F_{i} \rrbracket, \\
& T_{i}\left(E_{r}\right)=E_{r}, T_{i}\left(F_{r}\right)=F_{r} \quad(r \neq i, i \pm 1(\bmod N)) .
\end{aligned}
$$

Moreover the inverse $T_{i}^{-1}: \mathscr{D} U_{q}\left({ }^{s_{i}} \mathscr{E},{ }^{s_{i}} \Pi,{ }^{s_{i}} p,{ }^{s_{i}} \Gamma\right) \rightarrow \mathscr{D} U_{q}(\mathscr{E}, \Pi, p, \Gamma)$ satisfies:

$$
\begin{aligned}
& T_{i}^{-1}\left(K_{\alpha^{\prime}}\right)=K_{s_{i}\left(\alpha^{\prime}\right)}\left(\alpha^{\prime} \in^{s_{i}} P\right), T_{i}^{-1}\left(K_{\delta}^{+\frac{1}{2}}\right)=K_{\delta}^{+\frac{1}{2}}, \\
& T_{i}^{-1}\left(E_{i}\right)=-\bar{d}_{i+1} K_{i}^{-1} F_{i}, T_{i}^{-1}\left(F_{i}\right)=-\bar{d}_{i} E_{i} K_{i}, \\
& T_{i}^{-1}\left(E_{i-1}\right)=q^{-d_{i}}(-1)^{p\left(\alpha_{i}\right) p\left(\alpha_{i-1}\right)} \bar{d}_{i} \llbracket E_{i}, E_{i-1} \rrbracket, T_{i}^{-1}\left(F_{i-1}\right)=-\llbracket F_{i}, F_{i-1} \rrbracket, \\
& T_{i}^{-1}\left(E_{i+1}\right)=q^{-d_{i+1}} \bar{d}_{i+1} \llbracket E_{i}, E_{i+1} \rrbracket, T_{i}^{-1}\left(F_{i+1}\right)=-(-1)^{p\left(\alpha_{i}\right) p\left(\alpha_{i+1}\right)} \llbracket F_{i}, F_{i+1} \rrbracket, \\
& T_{i}^{-1}\left(E_{r}\right)=E_{r}, T_{i}^{-1}\left(F_{r}\right)=F_{r} \quad(r \neq i, i \pm 1(\bmod N)) .
\end{aligned}
$$

Define $Z_{i}: \mathscr{D} U_{q}(\mathscr{E}, \Pi, p, \Gamma) \rightarrow \mathscr{D} U_{q}\left({ }^{t_{i}} \mathscr{E},{ }^{t_{i}} \Pi,{ }^{t_{i}} p,{ }^{t_{i}} \Gamma\right)$ by $Z_{i}\left(K_{\alpha}\right)=K_{t_{t}(\alpha)}, Z_{i}\left(E_{j}\right)=$ $E_{i+j}$ and $Z_{i}\left(F_{j}\right)=F_{i+j}$.

Lemma 8.2.2. The isomorphisms $T_{i}$ 's and $Z_{j}$ 's satisfy the same equalities as in (8.1.2-3) with $T_{i}$ 's and $Z_{j}$ 's in place of $s_{i}$ 's and $t_{j}$ 's.

This can be checked directly.

For a parity preserving lattice isometry $w: P \rightarrow{ }^{w} P$ with a reduced expression $w$ $=t_{k} s_{i(1)} \cdots s_{i(a)}$, put $T_{w}=Z_{k} T_{i(1)} \cdots T_{i(a)}: \mathscr{D} U_{q}(\mathscr{E}, \Pi, p, \Gamma) \rightarrow \mathscr{D} U_{q}\left({ }^{w} \mathscr{E},{ }^{w} \Pi,{ }^{w} p,{ }^{w} \Gamma\right)$. By Lemma 8.2.2, $T_{w}$ is well-defined.

Lemma 8.2.3. Let $w: P \rightarrow{ }^{w} P$ be as above. Let $\alpha_{i} \in \Pi$. Assume $w\left(\alpha_{i}\right)=\alpha_{j}^{\prime} \in{ }^{w} \Pi$ where $\alpha_{j}^{\prime}$ denotes the $j$-th element of ${ }^{w} \Pi$. Then $T_{w}\left(E_{i}\right)=E_{j}$ and $T_{w}\left(F_{i}\right)=F_{j}$.

This can be checked directly (see also $[\mathrm{B}]$ ). 
By Lemma 6.3.1, there is a C-algebra anti-automorphism $\Omega: \mathscr{D} U_{q} \rightarrow \mathscr{D} U_{q}$ such that

$$
\Omega\left(E_{i}\right)=\bar{d}_{i+1} F_{i}, \Omega\left(F_{i}\right)=\bar{d}_{i+1} E_{i}, \Omega\left(K_{\alpha}\right)=K_{\alpha}^{-1}, \Omega\left(K_{\delta}^{+\frac{1}{2}}\right)=K_{\delta}^{\mp} \frac{1}{2}, \Omega(q)=q^{-1}
$$

Then we have $\Omega T_{w}=T_{w} \Omega$.

8.3. Let $\omega_{i}$ as in (8.1.4). We note:

Lemma 8.3.1. The elements $K_{\alpha}(\alpha \in P), K_{\delta}^{ \pm \frac{1}{2}}, T_{\omega_{i}}^{m}\left(E_{i}\right), T_{\omega_{i}}^{m}\left(F_{i}\right)(1 \leq i \leq N-1)$ generate the $\mathrm{C}(q)$-algebra $\mathscr{D} U_{q}$.

For $1 \leq i \leq n, k>0$ and $s \in \mathbf{Z}$, let

$$
\bar{\phi}_{i k}^{(s)}=K_{\delta}^{-\frac{k}{2}} q^{-\left(\alpha_{i}, \alpha_{i}\right)} \llbracket T_{\omega_{i}}^{s}\left(E_{i}\right), T_{\omega_{i}}^{k+s}\left(K_{i}^{-1} F_{i}\right) \rrbracket
$$

Note $\bar{\phi}_{i k}^{(s+m)}=T_{\omega_{i}}^{m}\left(\bar{\phi}_{i k}^{(s)}\right)$. Since $K_{i}^{-1} F_{i} \sim T_{i}^{-1} E_{i}$, by [L1, Lemma 40.1.2], we have $K_{\delta}^{\frac{k}{2}} \bar{\phi}_{i k}^{(s)} \in U_{q}^{+}$when $s \leq 0$ and $k+s>0$. Put $Q_{i j, k}=\frac{q^{k\left(\alpha_{i}, \alpha_{j}\right)}-q^{-k_{\left(\alpha_{i}, \alpha_{j}\right)}}}{q-q^{-1}}$ and $b_{i j}=$ $q^{\left(\alpha_{i}, \alpha_{j}\right)} K_{\delta}^{-\frac{1}{2}}$. By the same argument as in [B], we have

Lemma 8.3.2. (i) Assume $p\left(\alpha_{i}\right)=0$. Let $r>0$ and $m \in \mathbb{Z}$. Then $\bar{\phi}_{i r}^{(s)}=\bar{\phi}_{i r}^{\left(s^{\prime}\right)}$ $\left(s, s^{\prime} \in \mathbb{Z}\right)$, and we have:

$$
\begin{aligned}
{\left[\bar{\phi}_{i r}^{(0)}, T_{\omega_{i}}^{m}\left(F_{i}\right)\right]=} & -K_{\delta}^{\frac{1}{2}} Q_{i i, 1}\left\{\left(\left(q-q^{-1}\right) \sum_{k=1}^{r-1} b_{i i}^{1-k} T_{\omega_{i}}^{m+k}\left(F_{i}\right) \bar{\psi}_{i, r-k}^{(0)}\right)\right. \\
& \left.+b_{i i}^{1-r} T_{\omega_{i}}^{m+r}\left(F_{i}\right)\right\}
\end{aligned}
$$

and

$$
\begin{aligned}
{\left[\bar{\phi}_{i r}^{(0)}, T_{\omega_{i}}^{m}\left(E_{i}\right)\right]=} & K_{\delta}^{-\frac{1}{2}} Q_{i i, 1}\left\{\left(\left(q-q^{-1}\right) \sum_{k=1}^{r-1} b_{i i}^{k-1} T_{\omega_{i}}^{m-k}\left(E_{i}\right) \bar{\phi}_{i, r-k}^{(0)}\right)\right. \\
& \left.+b_{i i}^{r-1} T_{\omega_{i}}^{m-r}\left(E_{i}\right)\right\} .
\end{aligned}
$$

(ii) Assume $1 \leq i \neq j \leq n$. Let $r>0$ and $m \in \mathbb{Z}$. Then we have:

$$
\begin{aligned}
{\left[\bar{\phi}_{i r}^{(s)}, T_{\omega_{i}}^{m}\left(F_{j}\right)\right]=} & K_{\delta}^{\frac{1}{2}} Q_{i j, 1}\left\{\left(\left(q-q^{-1}\right) \sum_{k=1}^{r-1}\left(-b_{i j}\right)^{1-k} T_{\omega_{j}}^{m+k}\left(F_{j}\right) \bar{\phi}_{i, r-k}^{(s)}\right)\right. \\
& \left.+\left(-b_{i j}\right)^{1-r} T_{\omega_{j}+r}^{m+r}\left(F_{j}\right)\right\}
\end{aligned}
$$

and 


$$
\begin{aligned}
{\left[\bar{\phi}_{i r}^{(s)}, T_{\omega_{j}}^{m}\left(E_{j}\right)\right]=} & -K_{\delta}^{-\frac{1}{2}} Q_{i j, 1}\left\{\left(\left(q-q^{-1}\right) \sum_{k=1}^{r-1}\left(-b_{i j}\right)^{k-1} T_{\omega_{j}}^{m-k}\left(E_{j}\right) \bar{\phi}_{i, r-k}^{(s+k)}\right)\right. \\
& \left.+\left(-b_{i j}\right)^{r-1} T_{\omega_{j}}^{m-r}\left(E_{j}\right)\right\}
\end{aligned}
$$

Lemma 8.3.3. Let $1 \leq i \leq n$ and $r \in \mathbf{Z}$. Then:

$$
\begin{aligned}
& \llbracket T_{\omega_{i}}^{m+r}\left(F_{i}\right), T_{\omega_{i}}^{m}\left(F_{i}\right) \rrbracket=-\llbracket T_{\omega_{i}}^{m+1}\left(F_{i}\right), T_{\omega_{i}}^{m+r-1}\left(F_{i}\right) \rrbracket, \\
& \llbracket T_{\omega_{i}}^{m}\left(E_{i}\right), T_{\omega_{i}}^{m+r}\left(E_{i}\right) \rrbracket=-\llbracket T_{\omega_{i}}^{m+r-1}\left(E_{i}\right), T_{\omega_{i}}^{m+1}\left(E_{i}\right) \rrbracket .
\end{aligned}
$$

Proof. If $\left(\alpha_{i}, \alpha_{i}\right) \neq 0$, the result can be proved as in [B, Lemma 3.13]. Suppose $\left(\alpha_{i}, \alpha_{i}\right)=0$. Then it suffices to prove

$$
\left[T_{\omega_{i}}^{m+r}\left(F_{i}\right), T_{\omega_{i}}^{m}\left(F_{i}\right)\right]=0
$$

and

$$
\left[T_{\omega_{i}}^{m+r}\left(E_{i}\right), T_{\omega_{i}}^{m}\left(E_{i}\right)\right]=0
$$

Let $j$ be such that $\left(\alpha_{i}, \alpha_{j}\right) \neq 0$. Assume $r>0$. We show (8.3.6) by induction on $r$. Assume $r=1$. By (8.3.4) and $T_{\omega_{i}}\left(F_{i}\right)^{2}=0$,

$$
\left[T_{\omega_{i}}^{m+1}\left(F_{i}\right), T_{\omega_{i}}^{m}\left(F_{i}\right)\right]=-\frac{1}{Q_{j i, 1}} K_{\delta}^{-\frac{1}{2}}\left[\left[\bar{\phi}_{j 1}^{(s)}, T_{\omega_{i}}^{m}\left(F_{i}\right)\right], T_{\omega_{i}}^{m}\left(F_{i}\right)\right]=0
$$

Assume $r>2$. Then we have:

$$
\begin{aligned}
& \quad\left[T_{\omega_{i}}^{m+r}\left(\boldsymbol{F}_{i}\right), T_{\omega_{i}}^{m}\left(\boldsymbol{F}_{i}\right)\right] \\
& \quad=-\frac{1}{Q_{j i, 1}} K_{\delta}^{-\frac{1}{2}}\left[\left[\bar{\phi}_{j 1}^{(s)}, T_{\omega_{i}}^{m+r-1}\left(F_{i}\right)\right], T_{\omega_{i}}^{m}\left(F_{i}\right)\right]=\left[T_{\omega_{i}}^{m+1}\left(F_{i}\right), T_{\omega_{\imath}}^{m+r-1}\left(F_{i}\right)\right]=0 .
\end{aligned}
$$

Similarly we have (8.3.7).

Q. E. D.

Lemma 8.3.4. Let $i$ be such that $1 \leq i \leq N-1$ and $\left(\alpha_{i}, \alpha_{i}\right)=0$. Then, for any $s, m \in \mathbf{Z}$, we have

$$
\left[\bar{\phi}_{i 1}^{(s)}, T_{\omega_{i}}^{m}\left(E_{i}\right)\right]=0 \quad \text { and } \quad\left[\bar{\phi}_{i 1}^{(s)}, T_{\omega_{i}}^{m}\left(F_{i}\right)\right]=0
$$

and

$$
\bar{\phi}_{i 1}^{(s)}=\bar{\phi}_{i 1}^{(0)} \quad(s \in \mathbf{Z}) .
$$


Proof. By using (8.3.6), we can easily show

$$
\left[\bar{\psi}_{i 1}^{(0)}, E_{i}\right]=0 \quad \text { and } \quad\left[\bar{\psi}_{i 1}^{(0)}, F_{i}\right]=0 .
$$

Let $j$ be such that $1 \leq j \leq N-1$ and $\left(\alpha_{i}, \alpha_{j}\right) \neq 0$. By (8.3.5), we have

$$
\begin{aligned}
T_{\omega_{i}}^{-1}\left(\bar{\phi}_{i 1}^{(0)}\right) & =\bar{\phi}_{i 1}^{(-1)} \\
& =K_{\delta}^{-\frac{1}{2}}\left[T_{\omega_{i}}^{-1}\left(E_{i}\right), K_{i}^{-1} F_{i}\right] \\
& =K_{\delta}^{-\frac{1}{2}} Q_{j i, 1}^{-1} K_{\delta}^{-\frac{1}{2}}\left[\left[\bar{\phi}_{j 1}^{(0)}, E_{i}\right], K_{i}^{-1} F_{i}\right] \\
& =Q_{j i, 1}^{-1} K_{i}^{-1} \cdot Q_{j i, 1}\left[K_{\delta}^{\frac{1}{2}} T_{\omega_{i}}\left(F_{i}\right), E_{i}\right] \\
& =K_{\delta}^{-\frac{1}{2}}\left[T_{\omega_{i}}\left(K_{i}^{-1} F_{i}\right), E_{i}\right] \\
& =\bar{\phi}_{i 1}^{(0)} .
\end{aligned}
$$

Hence we have (8.3.9). By (8.3.9) and (8.3.10), we have (8.3.8). Q. E. D.

Lemma 8.3.5. Let $i$ be such that $1 \leq i \leq N-1$, and $r>0$. Then we have

$$
\begin{gathered}
{\left[\bar{\phi}_{k 1}^{(0)}, \bar{\phi}_{i r}^{(0)}\right]=0(1 \leq k \leq N-1),} \\
\bar{\phi}_{i r}^{(s)}=\bar{\phi}_{i r}^{(0)}(s \in \mathbf{Z}) .
\end{gathered}
$$

and

$$
\left[\bar{\phi}_{i r}^{(0)}, T_{\omega_{i}}^{m}\left(E_{i}\right)\right]=0 \quad \text { and } \quad\left[\bar{\phi}_{i r}^{(0)}, T_{\omega_{i}}^{m}\left(F_{i}\right)\right]=0 \quad\left(\left(\alpha_{i}, \alpha_{i}\right)=0\right)
$$

Proof. If $\left(\alpha_{i}, \alpha_{i}\right) \neq 0$, the statements follow from [B, Proposition 2].

We assume $\left(\alpha_{i}, \alpha_{i}\right)=0$. Let $j, 1 \leq j \leq N-1$, be such that $\left(\alpha_{i}, \alpha_{j}\right) \neq 0$. We use induction on $r$. For $r=1$, the statements immediately follow from Lemma 8.3.4.

We assume that the statements with $r-1$ in place of $r$ hold. By Lemma 8.3.2, Lemma 8.3.4, we have

$$
\begin{aligned}
T_{\omega_{i}}^{-1}\left(\bar{\phi}_{i r}^{(0)}\right) & =\bar{\phi}_{i r}^{(-1)} \\
& =K_{\delta}^{-\frac{r}{2}}\left[T_{\omega_{i}}^{-1}\left(E_{i}\right), T_{\omega_{i}}^{r-1}\left(K_{i}^{-1} F_{i}\right)\right] \\
& =K_{\delta}^{-\frac{r}{2}} Q_{j i, 1}^{-1} K_{\delta}^{\frac{1}{2}}\left[\left[\bar{\phi}_{j 1}^{(0)}, E_{i}\right], T_{\omega_{i}}^{r-1}\left(K_{i}^{-1} F_{i}\right)\right] \\
& =Q_{j i, 1}^{-1} K_{\delta}^{\frac{1-r}{2}}\left\{\left[\bar{\phi}_{j 1}^{(0)}, K_{\delta}^{\frac{r-1}{2}} \bar{\phi}_{i r-1}^{(0)}\right]+Q_{j i, 1}\left[K_{\delta}^{-\frac{1}{2}} T_{\omega_{i}}^{r}\left(K_{i}^{-1} F_{i}\right), E_{i}\right]\right\} \\
& =\bar{\phi}_{i r}^{(0)} .
\end{aligned}
$$

Hence the statement (8.3.12) holds. By $E_{i}^{2}=F_{i}^{2}=0$ (see Proposition 6.7.1), we 
have $\left[\bar{\phi}_{i a}^{(0)}, E_{i}\right]=0$ and $\left[\bar{\phi}_{i a}^{(0)}, T_{\omega_{i}}^{a}\left(F_{i}\right)\right]=0 . \quad$ By $(8.3 .12)$, we have

$$
\left[\bar{\phi}_{i a}^{(0)}, T_{\omega_{i}}^{m}\left(E_{i}\right)\right]=0 \quad \text { and } \quad\left[\bar{\phi}_{i a}^{(0)}, T_{\omega_{i}}^{m}\left(F_{i}\right)\right]=0
$$

where $1 \leq a \leq r$ and $m \in \mathbf{Z}$ (see also Lemma 2.2.1 (iii)). By Lemma 8.3.2 and (8.3.14), for any $k$ and any $u$, we have

$$
\left[\left[\bar{\phi}_{k 1}^{(0)}, \bar{\phi}_{i r}^{(0)}\right], T_{\omega_{u}}^{m}\left(E_{u}\right)\right]=0 \quad \text { and } \quad\left[\left[\bar{\phi}_{k 1}^{(0)}, \bar{\phi}_{i r}^{(0)}\right], T_{\omega_{u}}^{m}\left(F_{u}\right)\right]=0
$$

By Proposition 6.5.1 and Lemma 8.3.1, we have $\left[\bar{\phi}_{k 1}^{(0)}, \bar{\phi}_{i r}^{(0)}\right]=0 . \quad$ Q. E. D.

We put $\bar{\phi}_{i r}=\bar{\phi}_{i r}^{(0)}$. By Lemma 8.3.5, we have $T_{\omega_{i}}\left(\bar{\phi}_{i r}\right)=\bar{\phi}_{i r}$.

Lemma 8.3.6. For $1 \leq i, j \leq N-1$ and $r, 1>0$

$$
\left[\bar{\phi}_{k l}, \bar{\phi}_{i r}\right]=0 .
$$

Proof. We may assume $k<r$. We use induction on $l$. For $l=1$, the statement follows directly from Lemma 8.3.5. For general $l$, by Lemma 8.3.2 and Lemma 8.3.5, we have

$$
\left[\left[\bar{\phi}_{k l}, \bar{\phi}_{i r}\right], T_{\omega_{u}}^{m}\left(E_{u}\right)\right]=0 \text { and }\left[\left[\bar{\phi}_{k l}, \bar{\phi}_{i r}\right], T_{\omega_{u}}^{m}\left(F_{u}\right)\right]=0
$$

for any $u, 1 \leq u \leq N-1$, and any $m \in \mathbf{Z}$. By Proposition 6.5.1 and Lemma 8.3.1, we have $\left[\bar{\phi}_{k l}, \bar{\phi}_{i r}\right]=0$.

Q. E. D.

8.4. Define $h_{i k} \in \mathscr{D} U_{q}(k>0)$ by the following generating function in $z$.

$$
\exp \left(\left(q-q^{-1}\right) \sum_{k=1}^{\infty} h_{i k} z^{k}\right)=1+\left(q-q^{-1}\right) \sum_{k=1}^{\infty}(-1)^{i k} \bar{\phi}_{i k} z^{k}
$$

By Lemma 8.3.2, Lemma 8.3.5 and Lemma 8.3.6, we have:

Lemma 8.4.1. Let $i, j$ be such that $1 \leq i, j \leq N-1, r, l \in \mathbf{Z}$, and $k>0$. Then we have

$$
\begin{aligned}
& {\left[h_{i r}, h_{j l}\right]=0,} \\
& {\left[h_{i k}, T_{\omega_{j}}^{m}\left(F_{j}\right)\right]=-\frac{1}{k} Q_{i j, k} K_{\delta}^{\frac{k}{2}}(-1)^{j k} T_{\omega_{j}}^{m+k}\left(F_{j}\right),} \\
& {\left[h_{i k}, T_{\omega_{j}}^{m}\left(E_{j}\right)\right]=\frac{1}{k} Q_{i j, k} K_{\delta}^{-\frac{k}{2}}(-1)^{j k} T_{\omega_{j}}^{m-k}\left(E_{j}\right) .}
\end{aligned}
$$


Note $K_{\delta}^{\frac{k}{2}} h_{i k} \in U_{q}^{+}$.

Proposition 8.4.2. If $\sum_{i=1}^{N} \bar{d}_{i}=0$, then we have the following equalities:

$$
\sum_{i=1}^{n}\left[\sum_{j=1}^{i} k \bar{d}_{j}\right] K_{\delta}^{\frac{k}{2}} h_{i k}=0 \quad(k>0) .
$$

This follows directly from Lemma 8.4.1 and Proposition 6.5.1.

Put $h_{i,-k}=\Omega\left(h_{i k}\right)(k>0)$. By the equalities (QS6), we have the equalities

$$
\sum_{i=1}^{n}\left[\sum_{j=1}^{i} k \bar{d}_{j}\right] K_{\delta}^{-\frac{k}{2}} h_{i,-k}=0 \quad(k>0) .
$$

Theorem 8.4.3. Let $(\mathscr{E}, \Pi, p, \Gamma) \in \widetilde{\mathrm{AA}}(N)(N \geq 4)$ with $\sum_{i=1}^{N} \bar{d}_{i}=0$. Then $U_{q}(\mathscr{E}, \Pi, p, \Gamma)$ can also be defined by generators $K_{\alpha}(\alpha \in P), E_{i}, F_{i}(1 \leq i \leq N-1)$ and relations:

$$
\begin{gathered}
\text { (QS1), (QS2), (QS3), } \\
(\mathrm{QS4})(a)(a=1,2,3,4),(\mathrm{QS5})(a)(a=1,2,3,4)
\end{gathered}
$$

and

$$
\text { (QS6), (QS7). }
$$

Using Proposition 8.4.1, the statement is obtained by exactly the same argument as in the proof of Theorem 6.8.2.

8.5. For $1 \leq i \leq N-1$ and $r \geq 0$, put

$$
\phi_{i r}= \begin{cases}\left(q-q^{-1}\right) K_{i}(-1)^{i r} \bar{\phi}_{i r} & (r>0), \\ K_{i} & (r=0) .\end{cases}
$$

and $\varphi_{i r}=\Omega\left(\phi_{i r}\right)$. For $1 \leq i \leq N-1$ and $k \in \mathbf{Z}$, put $x_{i k}^{-}=(-1)^{i k} T_{\omega_{i}}^{k}\left(F_{i}\right)$ and $x_{i k}^{+}=$ $(-1)^{i k} T_{\omega_{i}}^{-k}\left(E_{i}\right)$. Let $\hat{\Gamma}=\Gamma+\frac{1}{2} \mathbf{Z} \delta$ (see 6.7). We denote the algebra of (8.2.1) by $\hat{U}_{q}(\mathscr{E}, \Pi, p, \Gamma)$. Using the same argument as in [B], we have

Theorem 8.5.1. Let $(\mathscr{E}, \Pi, p, \Gamma) \in \widetilde{\mathrm{AA}}(N)$ with $N \geq 3$. Then $\hat{U}_{q}(\mathscr{E}, \Pi, p, \Gamma)$ can also be defined by generators $\left\{K_{\alpha}(\alpha \in \hat{\Gamma}), K_{\delta}^{ \pm \frac{1}{2}}, x_{i j}^{ \pm}, h_{i k}\right\}$ and relations:

$$
\begin{gathered}
K_{0}=1, \quad K_{\alpha} K_{\beta}=K_{\alpha+\beta}, \\
K_{\alpha} x_{j k}^{ \pm} K_{\alpha}^{-1}=q^{\left( \pm \alpha_{j}+k \delta, \alpha\right)} x_{j k}^{ \pm},
\end{gathered}
$$




$$
\begin{gathered}
{\left[h_{i k}, h_{j l}\right]=\delta_{k,-l} \frac{1}{k} Q_{i j, k} \frac{K_{\delta}^{k}-K_{\delta}^{-k}}{q-q^{-1},}} \\
\sum_{i=1}^{n}\left[\sum_{j=1}^{i} k \bar{d}_{j}\right] K_{\delta}^{\frac{k}{2}} h_{i, k}=0(k \neq 0) \quad \text { if } \sum_{i=1}^{N} \bar{d}_{i}=0, \\
{\left[h_{i k}, x_{j l}^{ \pm}\right]= \pm \frac{1}{k} Q_{i j, k} K_{\delta}^{\mp \frac{|k|}{2}} x_{j, k+l}^{ \pm},} \\
x_{i k+1}^{ \pm} x_{j l}^{ \pm}-(-1)^{p\left(\alpha_{i}\right) p\left(\alpha_{j}\right)} q^{ \pm\left(\alpha_{i}, \alpha_{j}\right)} x_{j l}^{ \pm} x_{i k+1}^{ \pm}=(-1)^{p\left(\alpha_{i}\right) p\left(\alpha_{j}\right)} q^{ \pm\left(\alpha_{i}, \alpha_{j}\right)} x_{i k}^{+} x_{j l+1}^{ \pm}-x_{j l+1}^{ \pm} x_{i k}^{+}, \\
{\left[x_{i k}^{+}, x_{j l}^{-}\right]=\delta_{i j} \frac{K_{\delta}^{\frac{k-l}{2}} \varphi_{i k+l}-K_{\delta}^{\frac{l-k}{2}} \varphi_{i k+l}}{q-q^{-1}},}
\end{gathered}
$$

(In the following equations, $\operatorname{Sym}_{k_{1}, k_{2}}, \ldots, k_{s}$ denotes symmetrization with respect to $\left\{k_{1}\right.$, $\left.k_{2}, \ldots, k_{s}\right\}$.)

$$
\begin{aligned}
& \operatorname{Sym}_{k_{1}, k_{2}} \llbracket x_{i k_{1}}^{+}, \llbracket x_{i k_{2}}^{+}, x_{j l}^{ \pm} \rrbracket \rrbracket=0 \quad \text { if }\left(\alpha_{i}, \alpha_{i}\right) \neq 0 \text { and }\left(\alpha_{i}, \alpha_{j}\right) \neq 0 \text {, } \\
& \operatorname{Sym}_{k_{1}, k_{2}}\left[\llbracket\left[x_{i l}^{ \pm}, x_{j k_{1}}^{ \pm}\right], x_{u m}^{ \pm} \rrbracket, x_{j k_{2}}^{ \pm}\right]=0 \quad \text { if } \stackrel{i}{\times}-\stackrel{j}{\otimes}-\stackrel{u}{\times},
\end{aligned}
$$

Here $\phi_{i k}$ and $\varphi_{i k}$ are defined by the following equations.

$$
\begin{aligned}
& \sum_{k \geq 0} \varphi_{i k} z^{k}=K_{i} \exp \left(\left(q-q^{-1}\right) \sum_{r \geq 1} h_{i r} z^{r}\right), \\
& \sum_{k \geq 0} \varphi_{i k} z^{k}=K_{i}^{-1} \exp \left(\left(q^{-1}-q\right) \sum_{r \geq 1} h_{i,-r} z^{r}\right) .
\end{aligned}
$$

\section{Acknowledgments}

The author would like to thank Professor T. Tanisaki for explaining an unpublished result which was used for proving Proposition 6.5.1. He also thanks the referee for the careful reading and comments which were helpful in making the exposition more readable. A part of this work was done during the author's visit to the Department of Pure Mathematics and Mathematical Statistics at the University of Cambridge. He is most grateful to the staff of the Department, especially to Professor W. B. R. Lickorish, for hospitality.

\section{References}

$[B\rfloor$ Beck, J., Braid group action and quantum affine algebras, Comm. Math. Phys., 165 (1994), 555-568.

[D1] Drinfeld, V. G., Quantum groups, in Proc. Int. Congr. Math., Berkeley, 1986, Amer. Math. Soc., (1988), 798-820.

[D2] — On almost commutative Hopf algebras, Leningrad Math. J., 1 (1990), $321-342$. 
[D3] Drinfeld, V. G., New realizations of Yangian and quantum affine algebra, Sov. Math. Doklady, 36 (1988), 212-216.

[FSS] Frappet, L., Seiarino, A., and Sorba, P., Structure of basic Lie superalgebras and of their affine extensions, Comm. Math. Phys., 121 (1989), 457-500.

[GK] Gabber, O. and Kac, V. G., On defining relations of certain infinitedimensional Lie algebras, Bull. Amer. Math. Soc., 5 (1981), 185-189.

[IM] Iwahori, N. and Matsumoto, H., On some Bruhat decomposition and the structure of the Hecke rings of $p$-adic Chevalley groups, Publ. Math. IHES, 25 (1965), 5-48.

[J] Jimbo, M., A $q$-defference analogue of $\mathrm{U}(\mathrm{G})$ and the Yang-Baxter equation, Lett. Math. Phys., 10 (1985), 798-820.

[K1] Kac, V. G., Infinite dimensional Lie algebras, 3rd ed., Cambridge Univ. Press, Cambridge, 1990.

[K2] - Lie superalgebras, Adv. Math., 26 (1977), 8-96.

[KT] Khoroshkin, S. M. and Tolstoy, V. N., Twisting of quantum (super-)algebras, in Generalized symmetries in physics (Claustal, 1993), World Sci. Publishing, River Edge, NJ, (1994), 42-54.

[L1] Lusztig, G., Introduction to Quantum Groups, Birkhauser, 1993.

[L2] - Affine Hecke algebras and their graded version, Journal of AMS, 2 (1989), 599-625.

[LS] Leites, D., and Serganova, V., Defining relations for classical Lie superalgebras I, in Proc. Topological and Geometrical Methods in Field Theory, World Sci., Singapore, (1992), 194 $-201$.

[LSS] Leites, D., Saveliev, M., and Serganova, V., Embeddings of Lie superalgebra osp (1, 2) and nonlinear symmetric equations, in Group-theoretical methods in Physics, Proceedings of the Third Yurmala Seminar (Yurmala, 1995), Moscow, 1986, 255-298.

[R] Rosso, M., Finite dimensional representations of the quantum analog of a complex simple Lie algebra, Comm. Math. Phys., 117 (1988), 581-593.

[VdL1] Van De Leur, J. W., Contragredient Lie superalgebras of finite growth, Thesis, University of Utrecht, 1986.

[VdL2] - A classification of contragredient Lie superalgebras of finite growth, Comm. Algebra, 17 (1989), 1815-1842.

[S] Serganova, V., Automorphisms of simple Lie superalgebras, Math USSR-Izv., 24 (1984), 539-551.

[Y1] Yamane, H., Quantized enveloping algebras associated to simple Lie superalgebras and their universal R-matrices, Publ. RIMS, Kyoto Univ., 30 (1994), 15-87.

[Y2] - A Serre type theorem for affine Lie superalgebras and their quantized enveloping superalgebras, Proc. Japan Acad., 70A (1994), 31-36. 